\title{
A Magnetic Laser Scanner for Endoscopic Microsurgery
}

by

\author{
Alperen Acemoğlu
}

Department of Advanced Robotics,

Istituto Italiano di Tecnologia

Via Morego, 30, 16163 Genova, Italy

Department of Informatics, Bioengineering, Robotics and

Systems Engineering, Università degli Studi di Genova

Via All'Opera Pia, 13, 16145 Genova, Italy 



\title{
A Magnetic Laser Scanner for Endoscopic Microsurgery
}

\author{
by \\ Alperen Acemoğlu \\ Submitted to the \\ Department of Advanced Robotics, \\ Istituto Italiano di Tecnologia \\ and \\ the Department of Informatics, Bioengineering, \\ Robotics and Systems Engineering, \\ Università degli Studi di Genova \\ in partial fulfillment of the requirements for the degree of \\ Doctor of Philosophy \\ November 2017 \\ (c) Alperen Acemoğlu, 2017.
}

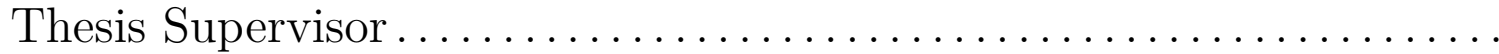

\section{Dr. Leonardo Serra Mattos}

Department of Advanced Robotics,

Istituto Italiano di Tecnologia

Via Morego, 30, 16163 Genoa, Italy 



\section{External Reviewers}

\section{Prof. Pietro Valdastri}

Chair in Robotics \& Autonomous Systems

School of Electronic and Electrical Engineering

University of Leeds

Leeds LS2 9JT, UK

\section{Dr.-Ing. Lüder Alexander Kahrs}

Head of Medical Technology Group

Institute of Mechatronic Systems

Leibniz Universität Hannover

Hannover, 30167, Germany 



\section{Preface}

The studies presented in this thesis were focused on designing, developing and characterizing a compact magnetic laser scanner for microsurgical applications and developing a laser incision depth controller to be used in soft tissue microsurgeries. Parts of these studies were presented in the following peer-reviewed publications written during the 3 years of the $\mathrm{PhD}$ program at the Istituto Italiano di Technologia:

\section{Journal Publications}

- Acemoglu, A., Deshpande, N., and Mattos, L. S., "Towards a Magnetically Actuated Laser Scanner for Endoscopic Microsurgeries", Accepted to be published in Journal of Medical Robotics Research, October 15, 2017.

- Acemoglu, A., Fichera, L., Kepiro, I. E., Caldwell, D. G., and Mattos, L. S., "Laser Incision Depth Control in Robot-Assisted Soft Tissue Microsurgery", Journal of Medical Robotics Research. (2017): 1740006.

\section{Conference Proceeding}

- Acemoglu, A., and Mattos L. S., "Magnetic Laser Scanner for Endoscopic Microsurgery", In Robotics and Automation (ICRA), 2017 IEEE International Conference on, pp. 4215-4220, IEEE.

\section{Workshop Abstracts}

- Acemoglu, A., Pucci, D., and Mattos L. S., "System Identification and FeedForward Control of a Magnetic Laser Scanner", Joint Workshop on New Technologies for Computer/Robot Assisted Surgery 7th edition, 14-15 September 2017, Montpellier, France.

- Acemoglu, A., Deshpande, N., and Mattos, L. S., "A Magnetic Laser Scanner for Non-Contact Endoscopic Ablations", Hamlyn Symposium on Medical Robotics, 25-28 June 2017, London, UK.

- Acemoglu, A. and Mattos, L.S., "Magnetically Actuated Surgical Laser Scanner for Endoscopic Applications", Joint Workshop on New Technologies for Computer/Robot Assisted Surgery 6th edition, 12-14 September 2016, Pisa, Italy.

- Acemoglu, A. and Mattos, L.S., "Characterization of Magnetic Field for Scanning Laser Module", Joint Workshop on New Technologies for Computer/Robot Assisted Surgery 5th edition, 10-12 September 2015, Brussels, Belgium. 



\title{
A Magnetic Laser Scanner for Endoscopic Microsurgery
}

by

\author{
Alperen Acemoğlu
}

\author{
Submitted to the \\ Department of Advanced Robotics, Istituto Italiano di Tecnologia and the \\ Department of Informatics, Bioengineering, Robotics and Systems Engineering, \\ Università degli Studi di Genova \\ on November 15, 2017, \\ in partial fulfillment of the requirements for the degree of \\ Doctor of Philosophy
}

\begin{abstract}
Laser scanners increase the quality of the laser microsurgery enabling fast tissue ablation with less thermal damage. Such technology is part of state-of-the-art freebeam surgical laser systems. However, laser scanning has not been incorporated to fiber-based lasers yet. This is a combination that has potential to greatly improve the quality of laser microsurgeries on difficult-to-reach surgical sites. Current fiberbased tissue ablations are performed in contact with the tissue, resulting in excessive thermal damage to healthy tissue in the vicinity of the ablated tissue. This is far from ideal for delicate microsurgeries, which require high-quality tissue incisions without any thermal damage or char formation. However, the possibility to perform scanning laser microsurgery in confined workspaces is restricted by the large size of currently available actuators, which are typically located outside the patient and require direct line-of-sight to the microsurgical area. Thus, it is desired to have the laser scanning feature in an endoscopic system to provide high incision quality in hard-to-reach surgical sites. This thesis aims to introduce a new endoscopic laser scanner to perform 2D position control and high-speed scanning of a fiber-based laser for operation in narrow workspaces. It also presents a technology concept aimed at assisting in incision depth control during soft-tissue microsurgery.

The main objective of the work presented in this thesis is to bring the benefits of free-beam lasers to laser-based endoscopic surgery by designing an end-effector module to be placed at the distal tip of a flexible robot arm. To this end, the design and control of a magnetic laser scanner for endoscopic microsurgeries is presented. The system involves an optical fiber, electromagnetic coils, a permanent magnet and optical lenses in a compact system for laser beam deflection. The actuation mechanism is based on the interaction between the electromagnetic field and the permanent magnets. A cantilevered optical fiber is bended with the magnetic field induced by the electromagnetic coils by creating magnetic torque on the permanent magnet. The magnetic laser scanner provides 2D position control and high-speed scanning of the
\end{abstract}


laser beam. The device includes laser focusing optics to allow non-contact incisions.

A proof-of-concept device was manufactured and evaluated. It includes four electromagnetic coils and two plano-convex lenses, and has an external diameter of 13 $\mathrm{mm}$. A $4 \times 4 \mathrm{~mm}^{2}$ scanning range was achieved at a $30 \mathrm{~mm}$ distance from the scanner tip. Computer-controlled trajectory executions demonstrated repeatable results with $75 \mu \mathrm{m}$ precision for challenging trajectories. Frequency analysis demonstrated stable response up to $33 \mathrm{~Hz}$ for $3 \mathrm{~dB}$ limit. The system is able to ablate tissue substitutes with a $1940 \mathrm{~nm}$ wavelength surgical diode laser.

Tablet-based control interface has been developed for intuitive teleoperation. The performance of the proof-of-concept device is analysed through control accuracy and usability studies. Teleoperation user trials consisting in trajectory-following tasks involved 12 subjects. Results demonstrated users could achieve an accuracy of $39 \mu \mathrm{m}$ with the magnetic laser scanner system.

For minimally invasive surgeries, it is essential to perform accurate laser position control. Therefore, a model based feed-forward position control of magnetic laser scanner was developed for automated trajectory executions. First, the dynamical model of the system was identified using the electromagnets current (input) and the laser position (output). Then, the identified model was used to perform feedforward control. Validation experiments were performed with different trajectory types, frequencies and amplitudes. Results showed that desired trajectories can be executed in high-speed scanning mode with less than $90 \mu \mathrm{m}$ (1.4 mrad bending angle) accuracy for frequencies up to $15 \mathrm{~Hz}$.

State-of-the-art systems do not provide incision depth control, thus the quality of such control relies entirely on the experience and visual perception of the surgeons. In order to provide intuitive incision depth control in endoscopic microsurgeries, the concept of a technology was presented for the automated laser incisions given a desired depth based on a commercial laser scanner. The technology aims at automatically controlling laser incisions based on high-level commands from the surgeon, i.e. desired incision shape, length and depth. A feed-forward controller provides (i) commands to the robotic laser system and (ii) regulates the parameters of the laser source to achieve the desired results. The controller for the incision depth is extracted from experimental data. The required energy density and the number of passes are calculated to reach the targeted depth. Experimental results demonstrate that targeted depths can be achieved with $\pm 100 \mu \mathrm{m}$ accuracy, which proves the feasibility of this approach. The proposed technology has the potential to facilitate the surgeon's control over laser incisions.

The magnetic laser scanner enables high-speed laser positioning in narrow and difficult-to-reach workspaces, promising to bring the benefits of scanning laser microsurgery to flexible endoscopic procedures. In addition, the same technology can be potentially used for optical fiber based imaging, enabling for example the creation of new family of scanning endoscopic OCT or hyperspectral probes. 


\section{Acknowledgments}

First and foremost, I would like to thank my supervisor Dr. Leonardo S. Mattos, Head of the Biomedical Robotics Laboratory. He has been always supportive during my $\mathrm{PhD}$ years. His enthusiasm on this research has motivated and encouraged me to keep working on this thesis. He guided me towards to correct answers in the most difficult times.

I would like to thank Dr. Nikhil Deshpande for his unfailing support and helps with fruitful discussions. I appreciate our conversations not only on research related topics but also about philosophical discussions on our existential reasons and personal development.

I would like to express my gratitude to Prof. Darwin G. Caldwell for giving me opportunity to be a part of Advanced Robotics Department at IIT.

I would like to give special thanks to Prof. Edward Grant for his support and suggestions during his IIT visits.

I am very grateful to external reviewers of this thesis Dr.-Ing. Lüder Alexander Kahrs and Prof. Pietro Valdastri for their support and invaluable feedback.

I would like to thank Dr. Loris Fichera for creating the basis of the laser incision depth control studies and giving me very useful feedback during the studies. I wish to thank Dr. Ibolya Kepiro for helping me in microscope examination of the tissue samples. I also would like to thank Dr. Daniele Pucci for his helps and support on feed-forward control for automated trajectory executions.

During my PhD years, I had chance to meet amazing people with whom I shared unforgettable memories. I thank all of my friends, especially: Zhuoqi Cheng, Matteo Rossi, Andrea S. Ciullo, Sara Moccia, Lucia Schiatti, Stefano Toxiri, Lorenzo Saccares, André Augusto Geraldes, Lorenzo Saccares, Olmo Alonso Moreno Franco, Tommaso Poliero, Matteo Sposito, Elif Hocaoğlu Çetinsoy, Dimitrios Kanoulas, Ioannis Sarakoglou, Theodore Tsesmelis, Joao Bimbo, Çigdem Beyan, Arman Savran, Sedat Dogan, Jorge Fernandez, Giacinto Barresi, Emidio Olivieri, Veronica Penza, and Jesus Ortiz. 
I am also grateful to the our department technicians Giuseppe Sofia, Marco Migliorini, Lorenzo Baccelliere, Riccardo Sepe, Francesco Di Dea, and Paolo Guria for their support and assistance. I would like to acknowledge the human resources and department secretaries, especially: Silvia Ivaldi, Valentina Rosso, Giulia Persano, Laura Galinta, Elisa Repetti, Floriana Sardi, and Stefano De Simone.

I am also very grateful to my previous supervisor Prof. Serhat Yeşilyurt. The working discipline that he taught during MSc studies helped me a lot to overcome the problems. I should express my gratitude to all my professors and teachers during all my education life.

I want to express my deepest gratitude to my family, who have always supported me especially in the hardest periods of my life. It would be really difficult without their emotional support. 
It is during our darkest moments that we must focus to see the light.

Aristotle 



\section{Contents}

$\begin{array}{ll}\text { Contents } & \text { Xv }\end{array}$

List of Figures $\quad$ xix

List of Tables $\quad$ XXV

1 Introduction 1

1.1 Laser Scanners . . . . . . . . . . . . . . . . . . . . . 1

1.1.1 Laser scanners for imaging . . . . . . . . . . . . . . . 1

1.1.2 Laser scanners in soft tissue microsurgeries . . . . . . . . . . . 3

1.2 Laser Microsurgery . . . . . . . . . . . . . . . . . . 6

1.2.1 Free-beam and fiber-based lasers . . . . . . . . . . . 6

1.2.2 The state-of-the-art of TLM . . . . . . . . . . . . 8

1.2.3 Endoscopic systems . . . . . . . . . . . . . . . . . . 9

1.2.4 Endoscopic systems in TLM . . . . . . . . . . . . . . 10

1.3 Problem Formulation . . . . . . . . . . . . . . . . . . . . 12

1.3 .1 Objectives ......................... 14

1.3.2 Thesis outline . . . . . . . . . . . . . . . 15

2 Magnetic Laser Scanner Design $\quad 19$

2.1 Conceptual Design . . . . . . . . . . . . . . . . . . . . . . . . . . 19

2.2 Prototype Manufacturing . . . . . . . . . . . . . . . . . . 20

2.3 Experimental Setup . . . . . . . . . . . . . . . . . 22

2.4 Teleoperation Control Setup . . . . . . . . . . . . . . 24 
2.5 Operating Modes . . . . . . . . . . . . . . . 25

2.6 Evaluation Methodology . . . . . . . . . . . . . . . . . 26

2.6 .1 Laser position detection $\ldots \ldots \ldots \ldots$

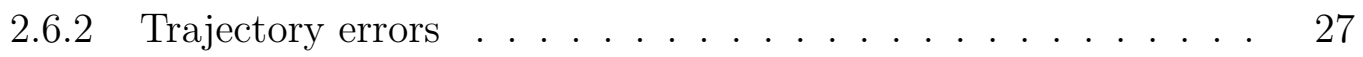

3 Characterization Experiments $\quad 29$

3.1 Workspace . . . . . . . . . . . . . . . . . . . . . . . . . 29

3.2 Repeatability . . . . . . . . . . . . . . . . . . 31

3.3 Step Response . . . . . . . . . . . . . . . . . . . . . . . . . . . . . 32

3.4 Frequency Response . . . . . . . . . . . . . . . . . . . . . 33

3.5 Deviation from linearity . . . . . . . . . . . . . . . 35

3.6 Preliminary Ablations Experiments . . . . . . . . . . . . . 36

4 Modeling, Identification and Feed-Forward Control 39

4.1 Modeling . . . . . . . . . . . . . . . . . . . . . . . . . . . 39

4.1.1 Magnetic actuation . . . . . . . . . . . . . . . . 39

4.1.2 Bending of a cantilevered optical fiber . . . . . . . . . 43

4.2 Identification and Feed-Forward Control . . . . . . . . . . . . . . 44

4.2 .1 Identification $\ldots \ldots \ldots \ldots \ldots \ldots$

4.2 .2 Feed-forward control . . . . . . . . . . . . . . . . . . 46

4.2 .3 Single target point . . . . . . . . . . . . . . 46

5 Teleoperation User Trials $\quad 49$

5.1 User Trials . . . . . . . . . . . . . . . . . . . . . . . . . . . . 49

5.1 .1 Subjective analysis . . . . . . . . . . . . 50

5.2 Results . . . . . . . . . . . . . . . . . . . . . . 52

5.3 Discussion . . . . . . . . . . . . . . . . . . . . . 57

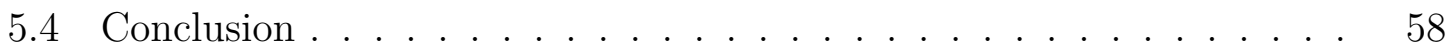

6 Automated Trajectory Executions $\quad 59$

6.1 2D Trajectory Executions $\ldots \ldots \ldots \ldots \ldots$

6.1 .1 Trajectory tracing . . . . . . . . . . . . 60 
6.1 .2 Scanning frequency $\ldots \ldots \ldots \ldots$

6.1 .3 Amplitude . . . . . . . . . . . . . . . . . . 61

6.1 .4 Hysteresis . . . . . . . . . . . . . . . . . 63

6.2 Discussion . . . . . . . . . . . . . . . . . . . 64

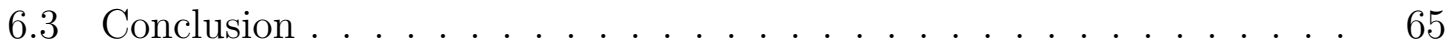

7 Laser Incision Depth Control $\quad 67$

7.1 Introduction . . . . . . . . . . . . . . 67

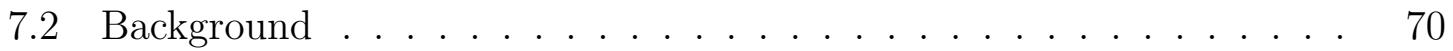

7.3 Modeling the Laser Incision Process . . . . . . . . . . . . . 72

7.3.1 Spatial energy distribution . . . . . . . . . . . . . 72

7.3.2 Feed-forward controller . . . . . . . . . . . . . . . . . . 74

7.4 Methodology . . . . . . . . . . . . . . . . . 75

7.4 .1 Experiments . . . . . . . . . . . . . . 75

7.4 .2 Materials and measurements . . . . . . . . . . . . . 77

7.5 Results . . . . . . . . . . . . . . . . . 80

7.5.1 Single-pass experiment . . . . . . . . . . . . 80

7.5.2 Multi-pass experiment . . . . . . . . . . . . 81

7.5.3 Computer-controlled laser incisions _ . . . . . . . . . 82

7.6 Discussion . . . . . . . . . . . . . . . . . . . . . . . . . . . . . . . 84

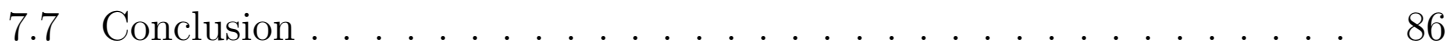

8 Discussion and Conclusion $\quad 89$

8.1 Discussion . . . . . . . . . . . . . . . . . . . . . 89

8.2 Conclusion . . . . . . . . . . . . . . . . . . . . . . . . . . 93

8.3 Future Research Directions . . . . . . . . . . . . . . . . . . . . 94

$\begin{array}{ll}\text { Bibliography } & 95\end{array}$ 



\section{List of Figures}

1-1 Basic components of a laser scanner for 3D imaging. . . . . . . . . . . 2

1-2 The state-of-the-art of Transoral Laser Microsurgery (TLM) setup. . 9

1-3 Thesis outline. . . . . . . . . . . . . . . . . . . . 16

2-1 Conceptual design of proposed magnetic laser scanner as an end-effector module of a flexible robotic arm. The magnetic laser scanner consists of an optical fiber, electromagnetic coils, a permanent magnet and optical lenses. The micromanipulation of the optical fiber is based on magnetic interaction between electromagnetic coils and the permanent magnet. . . . . . . . . . . . . . . . .

2-2 Prototype components (a) and assembled prototype (b) of magnetic laser scanner. . . . . . . . . . . . . . . . . . .

2-3 a) Experimental setup including the magnetic laser scanner, optical fiber, lenses, laser source and camera. b) Presentation of collimation and focusing of laser beam with two optical plano-convex lenses. . . .

2-4 Schematic illustration of the experimental setup. For simplicity, one degree of freedom is presented for magnetic actuation in the $\mathrm{x}$-axis. Laser spot position control on the computer screen with a tablet device is shown on the right side of the figure. . . . . . . . . . . .

2-5 Detection of laser spot in three steps: capturing the current frame (a), presenting the frame in red channel (b) and detection of the laser spot (c). Green rectangle presents the bounding rectangle of the laser spot. Red circle presents the centroid of the detected region. . . . . . . . 
2-6 Presentation of the trajectory error measurement with the target and executed trajectories. . . . . . . . . . . . . . . 28

3-1 Laser spot positions for different current values on the target surface. Black square in $[0,0]$ represents the reference point, i.e., the initial laser position on the target for currents $I_{y}=0$ and $I_{z}=0 . \quad \ldots . . \quad 30$

3-2 Current $(I)$ - displacement $(d)$ plots for y-axis (a) and z-axis (b). . . . 30

3-3 Repeatability results of the scan trajectories for 10 passes. All trajectories are overlapping and indistinguishable from each other. . . . . .

3-4 Repeatability results of ' $\mathrm{M}$ ' shaped trajectory for 10 passes in y-axis (a) and z-axis (b) . . . . . . . . . . . . . . .

3-5 Typical step response of the laser spot deflection for a current value of 0.165 A with settling time $\tau=0.7 \mathrm{~s}$, and overshoot percentage $P O=$ $89 \%$. Dashed red lines indicate the $2 \%$ range for the settling criterion. Settled response is highlighted with a green line. . . . . . . . . . . 33

3-6 Frequency response of the magnetic laser scanner. Magnitude $[\mathrm{dB}]$ excitation frequency $[\mathrm{Hz}]$ graph is presented. Red dashed-line shows 3 dB limit for cut-off frequency. . . . . . . . . . . . . . . . . . 34

3-7 a) A sample trajectory for RMSE calculation to demonstrate the deviation from linearity. b) Time-position graph for the sample trajectory.

3-8 Calculated RMSE for different excitation frequencies up to $100 \mathrm{~Hz}$. Average speed of the laser spot on the target was 1.44 and $144.2 \mathrm{~mm} / \mathrm{s}$ for 1 and $48 \mathrm{~Hz}$, respectively. . . . . . . . . . . . . . . 36

3-9 Experimental setup with the magnetic laser scanner and plaster block as a target. . . . . . . . . . . . . . . . .

3-10 Controlled ablations performed on plaster with $1940 \mathrm{~nm}$ diode laser. The different shapes show that any scan trajectory can be ablated with the magnetic laser scanner. . . . . . . . . . . . . . . . . . 
3-11 Typical 3D ablation profile produced on apple samples by the magnetic laser scanning tool when using the $1940 \mathrm{~nm}$ diode laser set to $2 \mathrm{~W}$ and 5 passes. Colorbar unit is in $\mu \mathrm{m}$. . . . . . . . . . . . . . . . 38

4-1 Presentation of the point $P$ according to the circular loop which carries current, I. . . . . . . . . . . . . . . . . . . . . . . . . . . . . 40

4-2 Schematically illustration of the magnetic actuation with four electromagnetic coils and permanent magnet. Electromagnetic coil pairs bend optical the fiber in $y$ - and z-axes with the effect of the magnetic torques $T_{y}$ and $T_{z}$. Corresponding beam bending angles are $\alpha_{z}$ and $\alpha_{y}$,

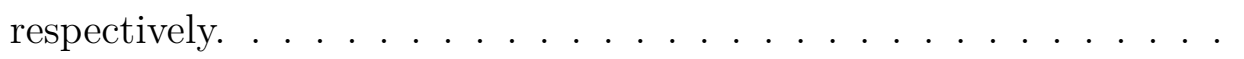

4-3 Calibration data set for the model estimation: current inputs are $u_{y}$ and $u_{z}$ in A, bending angles are $\alpha_{y}$ and $\alpha_{z}$ in mrad. . . . . . . . . 45

4-4 Block diagram of the feed-forward control. . . . . . . . . . . . . . . . 47

4-5 Executed actual trajectory for a sigmoid-shaped desired trajectory. . . 47

5-1 The target trajectories that are used in the experiments: $T_{1}$ and $T_{4}$ are s-curves, $T_{2}$ and $T_{5}$ are c-curves and $T_{3}$ is a straight trajectory. . . . . 50

5-2 A scene from user trials with the tablet control of magnetic laser scanner. 51

5-3 a) Comparison of a sample s-curve trajectory (red dots) against a target trajectory (black line). Green lines show the error bars for each measurement point. b) Calculated errors for all measurement points for the sample trajectory. . . . . . . . . . . . . . . .

5-4 The boxplots of the RMSE for each subject over 10 trials. The outliers are plotted individually with the red '+' symbol. . . . . . . . . . . . . 54

5-5 Comparison of the actual and estimated average execution times. . . 54

5-6 Overall average RMSE and maximum errors for each trial considering the data from all experimental subjects. . . . . . . . . . . 56

5-7 Average time for each trial over 12 subjects. . . . . . . . . . . 56

$5-8$ Questionnaire results . . . . . . . . . . . . . . 58 
6-1 Validation experiments for different trajectory types a) Circle, b) Square, and c) 8-shape. Error values for validation experiments are presented in Table 6.1. . . . . . . . . . . . . . . . . . . . 60

6-2 Validation experiments for different speeds with a) $1 \mathrm{~Hz}-2$ passes, b) $5 \mathrm{~Hz}-10$ passes, and c) $15 \mathrm{~Hz}-30$ passes. . . . . . . . . . . . . 61

6-3 Validation experiments with different amplitudes a) $\varnothing=1 \mathrm{~mm}, \mathrm{~b}$ ) $\varnothing$ $=2 \mathrm{~mm}, \mathrm{c}) \varnothing=3 \mathrm{~mm} \ldots \ldots \ldots \ldots$

6-4 2D straight trajectory in yz-plane for 8 passes in order to present system position response for loading and unloading conditions. . . . . .

7-1 a) The $\mu$ RALP surgical interface. The surgeon visualizes the surgical site through a stereoscopic display (HMD) while controlling the position of the laser beam using a stylus and graphics tablet. b) Technology concept. A virtual control element is superimposed to the surgeon interface, enabling an intuitive control of the laser incision depth. . . .

7-2 The motion of a the laser from a point $a$ to $b$ can be represented as a curve $\gamma \in \mathbb{R}^{2}$. . . . . . . . . . . . . . . . . . . . 74

7-3 The feed-forward controller scheme. . . . . . . . . . . . . .

7-4 Example of a depth map showing an incision crater produced on top of a tissue sample. This map has been reconstructed from microscopic images using the algorithm described by Aguet et al. Colorbar is in $\mu \mathrm{m} .79$

7-5 Example of Gaussian fitting. Estimated parameters are amplitude (a), mean $(\mu)$, standard deviation $(\sigma)$. For this particular example, $a_{1}=$ 392.7, $\mu_{1}=649.7, \sigma_{1}=219.1, a_{2}=2 \cdot 10^{6}, \mu_{2}=7649, \sigma_{2}=2000$. The parameter $a_{1}$ is taken as the depth of the crater. . . . . . . . 
7-6 Incision depth, $d[\mu \mathrm{m}]$, produced with different combination of laser power and speed in chicken muscle tissue (see Table 7.1). Results obtained with laser power $P=4 \mathrm{~W}$ are represented by empty box plots, while those for $P=8 \mathrm{~W}$ are represented by filled box plots. For a given value of laser power, the incision depth depends linearly on the energy density (for $4 \mathrm{~W}, d=181.2 \cdot e-73.8$ and for $8 \mathrm{~W}, d=211.9 \cdot e-48.7$ ).

7-7 Relative incision depth, $d / \overline{d_{s}}$ for different number of passes. $\overline{d_{s}}$ is average depth of the incisions produced with single pass. For all configurations, energy density, $e$, is fixed to $1.2 \mathrm{~J} / \mathrm{mm}^{2}$ and data are presented for $P=4 \mathrm{~W}-\overline{d_{s}}=143 \mu \mathrm{m}(\mathrm{a})$, and $P=8 \mathrm{~W}-\overline{d_{s}}=194 \mu \mathrm{m}(\mathrm{b})$. .

7-8 a) Incision depth, $d[\mu \mathrm{m}]$, for different energy densities, $e\left[\mathrm{~J} / \mathrm{mm}^{2}\right]$, and linear regression used to calculate the parameters for targeted depths.

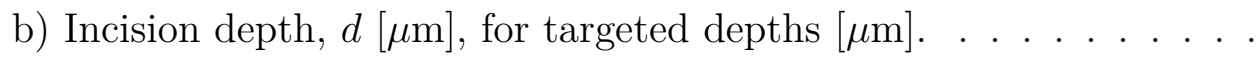

7-9 Comparison of depth maps of incisions performed with different energy densities and number of passes for targeted values; a) $300 \mu \mathrm{m}-[e=$ $\left.1.46 \mathrm{~J} / \mathrm{mm}^{2}, v=11.0 \mathrm{~mm} / \mathrm{s}, n=2\right]$, b) $500 \mu \mathrm{m}-\left[e=1.58 \mathrm{~J} / \mathrm{mm}^{2}, v\right.$ $=10.1 \mathrm{~mm} / \mathrm{s}, n=3]$, c) $800 \mu \mathrm{m}-\left[e=1.83 \mathrm{~J} / \mathrm{mm}^{2}, v=8.7 \mathrm{~mm} / \mathrm{s}, n\right.$ $=4]$. Colorbar is in $\mu \mathrm{m}$. . . . . . . . . . . . . . .

7-10 Histogram plot showing residual errors for the computer-controlled incision trials. . . . . . . . . . . . . . . . . . 



\section{List of Tables}

2.1 Prototype Components of Magnetic Laser Scanner . . . . . . . . . . . 21

5.1 Questionnaire Items . . . . . . . . . . . . . . . . . 52

6.1 RMSE values and maximum errors for validation experiments . . . . 62

7.1 Variance of incision depth produced with different combinations of laser

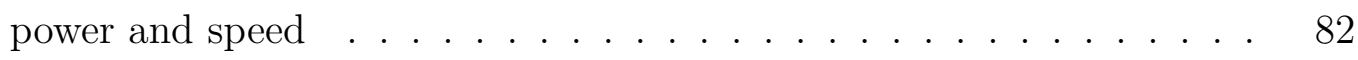

7.2 Results of the Controlled Incision Trials . . . . . . . . . . . . . . 84 



\section{Chapter 1}

\section{Introduction}

\subsection{Laser Scanners}

Laser scanners are optomechanical devices utilized for controlled deflection of a laser beam. The main application area of laser scanners is on imaging and three dimensional (3D) reconstruction of surfaces in mapping, architecture, optical microscopy, and diagnostics, etc. Laser scanners are versatile tools that are used also in laser cutting, marking, inscribing, 3D printing. Recently, they have become significant in soft tissue microsurgeries to improve the laser-tissue interaction. This section provides first an overview of laser scanners for imaging applications, then introduces laser scanners for soft tissue microsurgeries.

\subsubsection{Laser scanners for imaging}

Imaging and 3D reconstruction with laser scanners are achieved by collecting discrete distance measurements from a certain object or an environment. Each distance measurement corresponds to a point on the surface. A point cloud is created gathering all the points from the measurements. Then, 3D image of the surface is computed by building a mesh from the discrete point cloud.

Main components of a laser scanner for imaging are laser source, beam deflector, and position sensitive detector. Distance measurements are performed with triangu- 


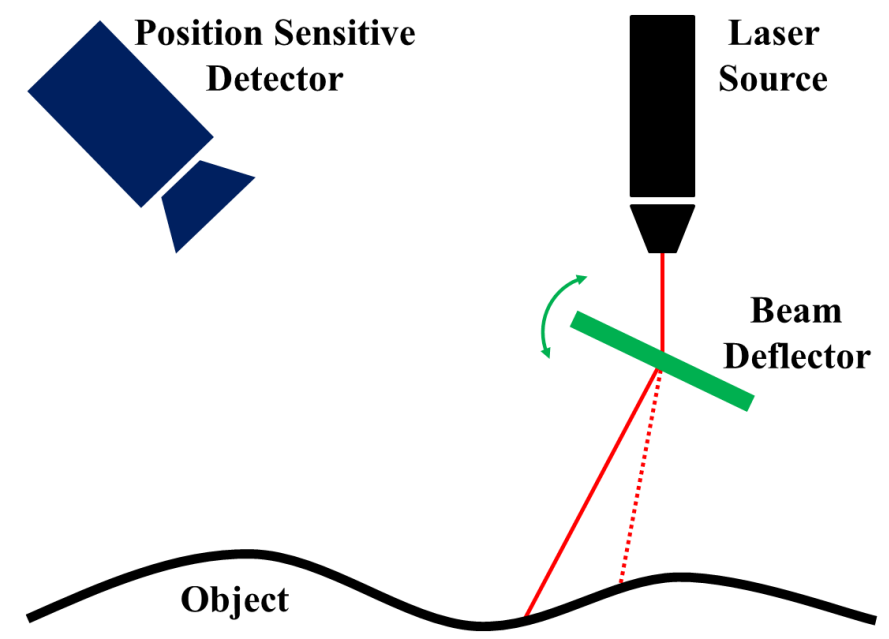

Figure 1-1: Basic components of a laser scanner for 3D imaging.

lation between the object, laser source and position sensitive detector (Fig. 1-1).

3D imaging with laser scanners has a wide range of applications including mapping of cities, imaging buildings, human bodies, biological samples. For example, for scanning large areas, airborne laser scanners were developed to be used for mapping roads, railways, damage assessment after natural disaster, and monitoring snow or ice covered areas, etc [1]. Also for 3D modeling and analysis of buildings, laser scanners became prevalent tools in architecture, engineering and construction [2]. Additionally, laser scanning technology improved the capabilities of medical imaging by providing high resolution $3 \mathrm{D}$ imaging, offering new possibilities for in vivo imaging.

\section{Laser scanners in medicine}

Lasers have been used in medicine for diagnoses, photochemotherapy, coagulation and welding of blood vessels, and laser surgery [3]. For medical imaging, laser scanners have even been proposed as an inexpensive alternative to the standard imaging systems such as computed tomography (CT) and magnetic resonance imaging (MRI) [4]. 3D reconstruction of outer surface of a human body can be performed by coupling a laser scanner with a robotic arm. When, surface 3D model of the body is required, 3D scanning technology can provide faster solutions than MRI, however imaging is limited to the body surface, internal part of the body can not be visualized. Another 
study shows that laser doppler scanner can be used to measure flow rate on the skin for the assessment of burn depth [5]. Moreover, 3D imaging with laser scanners has wide range of applications in dentistry to aid diagnosis and treatment planning [6]. Additionally, laser scanners with electromechanical vibrators were combined with optical microscopy to have better resolution and contrast for examination of a specimen in 1980 [7].

Imaging of internal body parts is possible with flexible optical fibers. Due to the different optical properties of healthy and malignant tissues, reflections from the tissues can be used for diagnostic purposes [3]. Researches on developing compact laser scanners for in vivo imaging have been mainly concentrated around (i) MEMS based mirror scanners and (ii) optical fiber scanners. Various MEMS based scanning mirrors have been developed for biomedical applications with electrostatic, electrothermal, and electromagnetic actuation [8]. Moreover, endoscopic optical fiber scanners have been proposed for in vivo imaging. For example, researchers developed a single fiber endoscope for 3D imaging by actuating the fiber externally with motor or galvanometer [9]. Additionally, a resonant fiber scanner with $1.06 \mathrm{~mm}$ diameter was proposed for imaging with piezoelectric actuation [10]. It was also reported that a fiber scanner with piezobender elements can be used for in vivo multiphoton imaging [11].

\subsubsection{Laser scanners in soft tissue microsurgeries}

Lasers have been commonly used in tissue microsurgeries for about 50 years. The development of $\mathrm{CO}_{2}$ laser in 1964 [12] brought an alternative approach to perform incisions on soft tissues. It has been demonstrated that a focused $\mathrm{CO}_{2}$ laser beam could be used for resections of the vocal cords with minimal bleeding in an experimental study on animals [13]. The $\mathrm{CO}_{2}$ laser has been incorporated to a microscope for the excision of a laryngeal carcinoma from a human patient by Strong (1975) providing a breakthrough in surgical procedures in clinical setting [14]. The advantage of using lasers in surgeries are the precise tissue removal when supplied with a microscope, minimal morbidity, reduced or no post-operative pain and cost-effectiveness compared to the conventional surgical techniques [15]. Similar results have been reported by 
White et al. on laser-based intraoral soft tissue surgery with less bleeding both during surgery and post-operatively compared to the conventional method performed with a scalpel [16].

Application of lasers in soft tissue surgery continues to grow since the early days due to many advantages such as the coagulation of small blood vessels while cutting, the sterilization of the wound, and the absence of post-operative edema. Lasers also offer a good alternative to radiation therapy for the treatment of laryngeal cancers $[17,18]$. A review by Milford et al. from 1991 provides a detailed comparitive study on radiotherapy and laser excision for the treatment of laryngeal carcinomas taking into account recurrence and total treatment period. The conclusion was a positive assessment of laser surgery as a valid alternative treatment [19]. Soon after, it was reported that laser surgery enables effective treatment of T1 and T2 type tumors in the larynx, and constitutes a convenient and acceptable alternative for the patient $[20]$.

After laser-based microsurgery became an accepted treatment as a better alternative to the traditional methods, thermal damage and tissue carbonization during tissue ablation appeared as a challenge for delicate operations. In 1993, researchers proposed surgery with laser scanning for char-free ablation in rectal surgery [21]. They reported that fast and homogeneous movement of the laser on the tissue minimizes thermal damage to the healthy tissue in the vicinity of the surgical site.

\section{Why do laser scanners improve ablation quality?}

In order to answer this question, requirements of the surgical precision must be examined for laser-based operations. Precise tissue ablation requires [22]:

- a short optical penetration depth and

- a short pulse duration

for a focused laser. Optical penetration depth depends on the absorption coefficient of the tissue at a specific wavelength. For short optical penetration depth, absorption coefficient must be high for the wavelength of the chosen laser. Thus, laser light 
is highly absorbed by the tissue, and ablation threshold is reached faster. On the other hand, short pulse duration is important for lateral thermal damage. During the ablation of a volume of a tissue, heat diffusion occurs towards to the non-ablated tissue. Thermal diffusion time is calculated as:

$$
t_{d}=\frac{1}{\kappa \mu_{a}^{2}}
$$

where $\kappa$ is the thermal diffusivity, and $\mu_{a}$ is the absorption coefficient [22]. Then, the thermal confinement condition can be expressed with a dimensionless parameter:

$$
t_{d}^{*}=\frac{t_{p}}{t_{d}}=\kappa \mu_{a}^{2} t_{p} \lesssim 1
$$

where $t_{p}$ is pulse duration. Thus, pulse duration must be smaller than the thermal diffusion time in order to minimize the heat diffusion from the ablated volume to the non-ablated tissue in the vicinity. If $t_{d}^{*}$ is greater than unity, a heat accumulation occurs for repetitive pulses, causes char formation and thermal damage. In order to minimize the extent of the thermal damage during the tissue ablations,

- laser on/off time can be regulated to change the pulse repetition rate or

- scanning of laser beam can be performed on the tissue

in order to increase the time between subsequent exposures. Thus, laser scanners ensure thermal confinement condition for relatively longer pulses by moving the laser beam on the tissue.

In today's practice, laser scanners are being used for resection of the laryngeal carcinoma in transoral laser microsurgery (TLM). It was proven that laser scanners allow high quality tissue ablations on vocal cord surgeries [23, 24]. These laser scanners have fast steering mirrors for the scanning of the free-beam $\mathrm{CO}_{2}$ lasers. Additionally, an endoscopic fiber scanner was developed with piezoelectric actuation for the tissue ablation on vocal cords [25]. The system was coupled to an ultrafast erbium-doped fiber laser, enabling the tissue ablations. Moreover, researchers developed a laser scanner with a $2 \mathrm{D}$ spatial disperser without any mechanical movement of the fiber or 
the sample [26]. Scanning was achieved by changing the wavelength of the emitted light. The system was proposed for precise surgeries to remove malignant tissues or treatment of movement disorders. This work contributes to these same goals by developing a magnetic laser scanner to be used as a tip module of an endoscopic system for delicate microsurgeries [27].

\subsection{Laser Microsurgery}

\subsubsection{Free-beam and fiber-based lasers}

Laser-based microsurgeries have recently been used in different medical operations such as transoral microsurgery, transurethral resections and refractive surgery. Good post-operative functions, low morbidity and minimal peripheral tissue injury are the main strengths of laser-based microsurgery over the conventional cold-steel surgery techniques [28, 29]. Laser-based surgeries provide significantly higher incision efficiency compared to the monopolar electrosurgery [30]. In addition to these, previous researches indicate that $\mathrm{CO}_{2}$ laser causes less thermal damage as compared to the harmonic ultrasonic scalpel $[31,32]$. The main two technologies allowing these advantages are: free-beam and flexible fiber-based laser ablation systems. In free-beam laser systems, generally a $\mathrm{CO}_{2}$ laser is coupled with a microscope and laser position is controlled with micromanipulator, which is a small joystick. It controls the laser position by changing the orientation of a beam deflection mirror. Free-beam laser systems have a scanning feature for high speed actuation of the laser with fast steering mirrors for better laser tissue interaction. Laser scanning feature ensures the soft tissue ablation quality with less or no carbonization by providing fast and continuous motion [23]. Although free-beam laser provide good ablation quality, they have compelling limitations in surgical practice [33].

Flexible fiber-based systems use optical fibers for diode lasers or flexible hollow tubes for $\mathrm{CO}_{2}$ lasers in order to deliver the laser close to the surgical site. With this

ability, accessing hard-to-reach surgical sites with a laser becomes possible through 
natural orifices or a single port. For example, feasibility study of endoscopic laser ablation in breast tissue showed that fiber-based thulium laser can be used as a minimally invasive treatment of intraductal papillomas [34]. In dentistry, root canal therapy was performed with an excimer laser utilizing different core diameter optical fibers [35]. Additionally, fiber-based lasers may contribute to shorten operating time compared to the free-beam laser systems [36]. Moreover, flexible $\mathrm{CO}_{2}$ lasers provide fine incisions, excellent hemostasis, and minimal peripheral tissue injury [37]. In order to bring these advantages to the surgical practice, developing compact laser ablation systems and couple them with endoscopic tools became an important research area recently. Researchers developed robotic platforms coupled with an optical fiber that carries the laser close to the surgical site. More precisely, a prototype fiber-based laser tool has been coupled to the daVinci S System to perform nerve-sparing radical prostatectomy using laser energy [38]. Similarly, in other studies, a flexible fiber has been coupled with the daVinci surgical robot arm to perform transoral surgery [28, 29]. Besides the possibility of coupling systems with commercial robotic platforms, researchers have also investigated manipulators to steer the laser light for medical operations performed in narrow workspaces. A bending laser manipulator has been developed for intrauterine fetal surgery and the feasibility of the usage of the system was discussed with in vitro and in vivo experiments [39]. Similarly, wire-guided bending mechanisms have been used to change the irradiation direction of the laser for photocoagulation in fetoscopic surgery [40].

Fiber-based ablations are performed in close proximity to tissue, since laser beam diverges when it exits the fiber. In other words, in order to perform efficient ablations, fiber is kept very close to the tissue (almost in contact with the tissue) not to lose laser power. Especially during manual control of the fiber in surgeries, fiber often touches the tissue due to hand tremors. Ablations in close proximity to the tissue can suffer from the problem of tissue residue sticking to the fiber, which reduces ablation quality and causes damage to the tip of the fiber. Additionally, since fiber-based systems do not offer scanning capabilities yet, they cause tissue carbonization in the vicinity of the surgical site. 


\subsubsection{The state-of-the-art of TLM}

In the field of laryngology, the state-of-the-art non-invasive treatment technique is a laser-based procedure called Transoral Laser Microsurgery (TLM). TLM is a suite of complex laryngological methods involving the precise micromanipulation of a surgical laser in a narrow workspace (larynx and vocal cords). The goal is to perform tumor resection trans-orally, thereby avoiding the problems of traditional open-neck surgeries performed with cold steel instruments [18]. Precise tissue ablations are needed to completely remove the malignant tissue while preserving healthy tissue around it.

In laryngology, free-beam lasers first became prevalent in the 1970's when the $\mathrm{CO}_{2}$ laser was coupled to a surgical microscope $[41,14]$. The surgical setup includes a laryngoscope, a device that establishes and maintains a direct line-of-sight to the larynx under a surgical microscope (Fig. 1-2). The surgical laser is controlled with a micromanipulator, which is a small joystick, attached to the microscope. This micromanipulator controls the orientation of a beam deflection mirror which in-turn positions the laser spot at the surgical site.

This surgical setup is effective but suffers from significant limitations in controllability and ergonomics [33]. In these systems, the typical operating distance is $400 \mathrm{~mm}$. This means that the microscope and the laser control device are located quite far from the surgical site, making the direct line-of-sight requirement during surgery highly challenging. The laryngoscope itself is a cumbersome device requiring the patient's head to be put in an awkward position, which can cause soft tissue lesion, bleeding, or post-operative pains [42]. Furthermore, proper usage of the micromanipulator is also highly dependent on the dexterity and experience of the surgeon [33, 43, 44]. Given these limitations, it is crucial to develop devices that can not only improve the delivery of the laser to the surgical site but also simplify the surgeon-machine interface while overcoming the challenges of controllability and ergonomics. The introduction of endoscopic surgery in TLM has the potential to address these limitations in a similar way as it has done in other procedures $[45,46]$. 


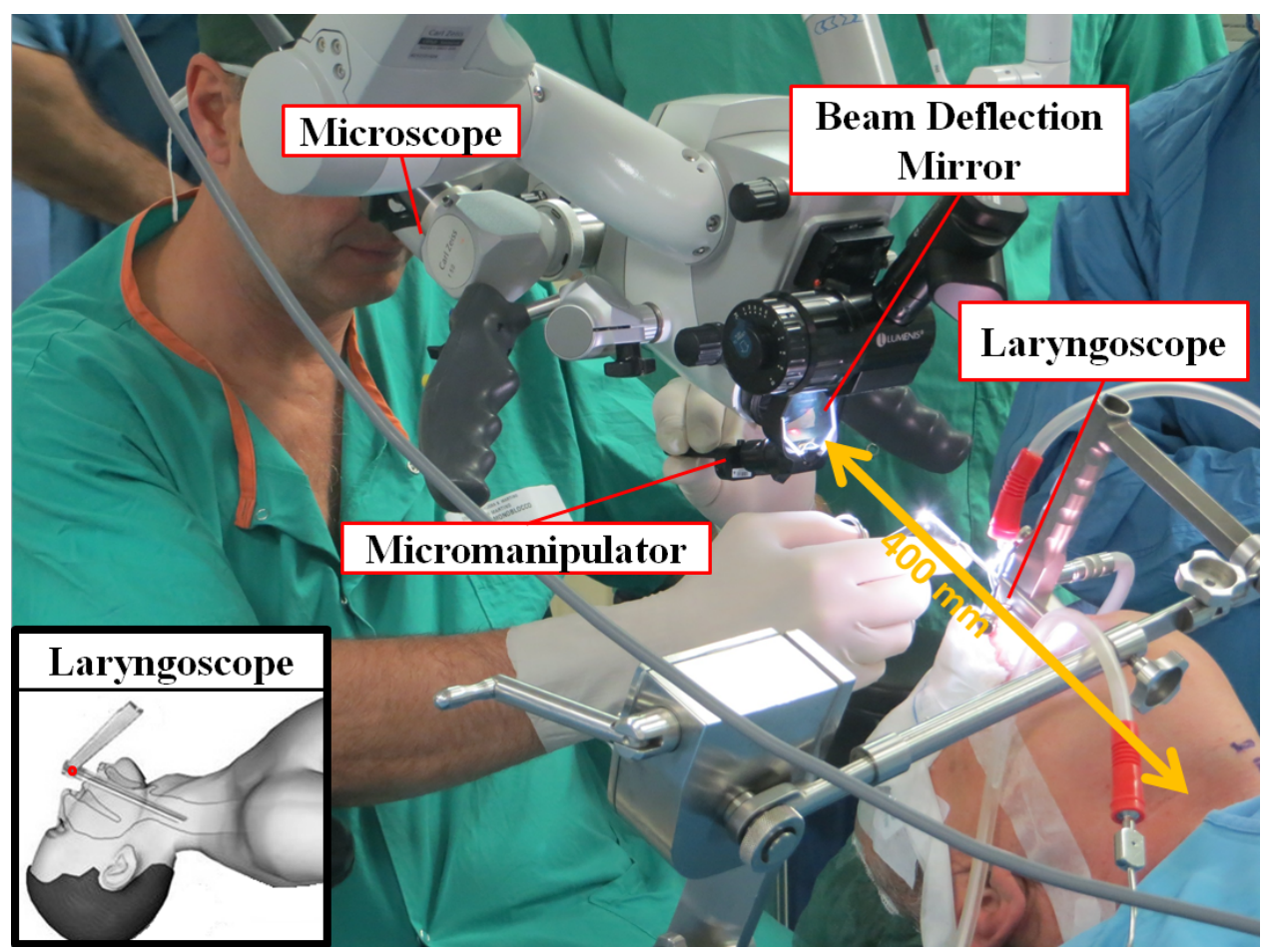

Figure 1-2: The state-of-the-art of Transoral Laser Microsurgery (TLM) setup.

\subsubsection{Endoscopic systems}

The introduction of surgical capability as well as computer-assisted features to diagnostic-only instruments, allowed endoscopy to be incorporated into surgical thinking [47]. Endoscopic surgical platforms are being utilized in various procedures including abdominal [47], transvaginal, [48] and trans-urethral [49] due to their advantages or few-to-no incisions and closer access to surgical site. Examples of these platforms include both manual and robotic systems: EndoSamurai from Olympus [50], DDES from Boston Scientific [51], MASTER system [52], the ViaCath system from Hansen Medical [53], and Anubis scope from Karl Storz [54].

Independently, the diagnostic capabilities of endoscopes have also been under investigation for augmentation. Researchers introduced optical fiber scanning systems with dimensions comparable to those of traditional endoscope for capturing images $[9$, 55]. A three-dimensional real-time imaging system using a single optical fiber that is scanned over the target area using an external mechanical-transduction mechanism 
is presented in [9]. A similar fiber positioning concept is used in the Cobra fiber positioner device [55]. Lee et al. discuss the scanning fiber endoscope that uses laserbased video imaging [56]. The design is based on custom piezo-electric actuators which position a cantilevered laser-light carrying fiber over the 2D imaging plane. Conceivably, this novel scanning fiber capability adapted to surgical lasers can provide a completely new approach to laser-based robotic endoscopic surgery [57, 45].

\subsubsection{Endoscopic systems in TLM}

Performing laser incisions closer to the surgical site has high importance due to the advantages they can offer over the state-of-the-art systems. Endoscopic systems can eliminate the direct line-of-sight requirement and enable access to parts of the surgical site that are not reachable with the traditional systems. Reducing the operating distance can also potentially improve the precision of the surgical operations [44]: Laser position control can be performed close to the target tissue rather than from 400 mm away. Additionally, with a design adapted to the patient's anatomy, endoscopic systems can eliminate requirement of the laryngoscope itself. On the other hand, the surgical microscope can be replaced with endoscopic cameras. These can provide simpler and more ergonomic visualization to the surgeons, and also introduce imagingassisted features [45]. Lastly, the manual micromanipulator can be replaced with more intuitive control methods, e.g., a tablet device, providing a comfortable surgeonmachine teleoperation interface.

The introduction of endoscopic surgical systems in laryngology has held interest in the research as well as commercial community. The FLEX Robotic System [58] is the first flexible and steerable endoscopic system cleared by FDA for use in transoral surgical procedures. It offers a diameter of $19 \mathrm{~mm}$ and includes two manually-controlled, interchangeable, instrument arms as well as a camera for visualization. Researchers at Vanderbilt University have demonstrated two independent laparo-endoscopic systems: the IREP system [59] and the Concentric Tube-based system [49]. The IREP offers integrated 3D vision within a $15 \mathrm{~mm}$ tube with two dexterous robotic arms with 6-DOF manipulation. The Concentric Tube-based robotic endoscopic system 
offers two continuum robot automated arms attached to a commercial endoscope for a small (dia. $8.66 \mathrm{~mm}$ ) semi-automated system. Additionally, a dual-arm snake-like robot was proposed for minimally invasive surgery of throat [60]. The complete system with two arms has 20 actuated joints and allows suturing and bi-manual knot tying. Although none of these systems are designed for laser-based microsurgeries, their instrument arms can be adapted to carry optical fiber to provide laser incisions in contact with tissue. Patel et al. [44] present another endoscopic tool designed for laryngeal resection using a $\mathrm{CO}_{2}$ laser. This system delivers laser beam through an optical fiber to the tip of a $17 \mathrm{~mm}$ diameter customized endoscope and laser position control is performed by refracting the laser beam using two optical wedges. They presented the capability of the tool with different trajectories indicating that system can provide repeatable performance [44].

Researchers proposed an endoscopic system coupled with a femtosecond laser for microsurgeries [25]. The system uses an erbium-doped fiber laser and the outer diameter of the device is $5 \mathrm{~mm}$. An optical fiber is scanned with piezoelectric actuation. The device provides variable ablation width $(3-220 \mu \mathrm{m})$ for tissue removal. Total scanning workspace is $200 \times 200 \mu \mathrm{m}$.

Most recently, the European project $\mu$ RALP has focused on developing an endoscopic laser scanning system for robot-assisted surgeries, enabling the replacement of the manual micromanipulator with a graphics tablet [57]. Within $\mu$ RALP, an endoscope with a bending distal end was designed with tendon driven actuation for the laser treatment of laryngeal cancer [61, 62]. Additionally, researchers presented an endoscopic tool consisting of a silicon-mirror based micromanipulation robot driven by two piezoelectric motors [63] to steer a laser beam emanating from an optical fiber carried to the tip of the endoscope [45]. This endoscopic tool has $18 \mathrm{~mm}$ diameter and includes a stereo vision system. They also presented the image-based control of the laser spot position by placing optical fiber bundles to capture the images. As yet, the research has not incorporated any laser focusing optics in the tool for non-contact laser incisions.

From this discussion, it is evident that a tool offering precise laser incisions in a 
small form-factor forms a critical component of any laser-based endoscopic surgical system. The research in this thesis takes its motivation from these previous research efforts to present a new endoscopic laser scanner device for robot-assisted endoscopic laser microsurgeries.

\subsection{Problem Formulation}

The introduction of an endoscopic surgical device in TLM would require not only addressing the limitations of state-of-the-art systems, but also incorporating the features that surgeons have come to expect from TLM surgical devices. Therefore, it is essential to examine in detail the features of the state-of-the-art TLM systems. As mentioned earlier, the current laser systems (e.g., Lumenis - Digital Acublade [64], Deka HiScan Surgical [65]) use a small manual micromanipulator, attached to a surgical microscope, to steer a free-beam laser spot on the target tissue. By design, this implies that the incisions are non-contact. The incorporation of a scanning system brings significant advantages with respect to quality of cut as well as thermal damage to and carbonization of surrounding tissue [23]. This feature guarantees high incision quality with minimum carbonization and thermal damage during soft tissue ablations. Current commercial systems have high-speed scanning as a standard feature $[64,65]$. In free-beam systems, the scanning of the laser is performed with fast steering mirrors.

Consequently, the design of the endoscopic tool should involve:

(i) delivery of laser to the surgical site while allowing non-contact incisions,

(ii) incorporating the feature of high-speed scanning for high incision quality,

(iii) improving the laser control interface, and

(iv) providing additional features for improved surgical performance.

As has been discussed earlier, optical fibers can carry laser light to the tip of endoscopes [56]. Optical fibers can be coupled with high-power $\mathrm{CO}_{2}$ and diode lasers 
to perform incisions in soft tissues $[66,67]$. This ability allows the lasers to access the surgical sites that are difficult-to-reach with conventional methods. In addition, fiberbased lasers may contribute to shorten the operating time of some procedures as compared to free-beam laser surgery, as demonstrated for the case of stapes surgery [36].

A limitation of fiber-based operations is that the incisions need to be performed in close proximity to the tissue, almost in contact $[49,58]$. This is because the laser light diverges as it exits the optical fiber. Additionally, existing fiber-based systems employ manual control of the fiber [44]. Due to the hand tremors, optical fibers often penetrate the tissue. The sticking of tissue residue to the fiber itself is a major problem, causing damage to the tip of the fiber and leading to reduced quality of the incision. It would therefore be beneficial to isolate the bare optical fiber from the surgical site to avoid the direct interaction with the tissue and thereby retain the benefits of non-contact laser incisions. To overcome the problem of laser light divergence, optical lenses can be used at the distal end of the fiber to focus the light on a target plane.

In order to enable the scanning feature in endoscopic applications, it is desirable to miniaturize the concept of the fast steering mirrors and adapt it to scanning the laser fiber itself. Investigating new actuation mechanisms for scanning and position control is crucial for the surgical operations in the narrow workspaces. Researchers have proposed magnetic actuation for the miniaturization of the surgical catheters $[68$, $69,70]$. In these studies, a permanent magnet attached to a cantilevered beam is actuated with an external magnetic field for position control. Magnetic fields allow high frequencies appropriate for the scanning feature. Furthermore, the actuated cantilever beam can be replaced with a cantilevered optical fiber thus keeping the same control strategy with magnetic field for changing the laser beam direction.

Earlier studies have shown that the control of the laser with a manual micromanipulator is highly challenging especially during long surgeries [71]. For a better control strategy, the tablet device control of the laser has been proposed providing a writing-like intuitive interface $[57,43,72]$. It has been shown that tablet device provides more intuitive and precise control for laser micromanipulation as compared to 
the traditional manual system. Thus, miniaturization and adaptation of the state-ofthe-art laser-based surgical systems can be done using the optical fibers and a tablet interface for endoscopic applications.

\subsubsection{Objectives}

Taking into consideration the challenges of the state-of-the-art systems discussed in the previous section, the objective with the proposed work is developing an endoscopic laser scanner for delicate and high-quality soft tissue ablations by bringing advantages of the free-beam and fiber-based laser systems together. Main design objectives are:

(i) bringing laser closer to the surgical site to access hard-to-reach surgical sites,

(ii) enabling high-speed scanning of the laser at the tip of an endoscope for high quality tissue ablations,

(iii) providing an intuitive laser position control with a compact actuation mechanism, and

(iv) focusing laser on the tissue using optical lenses enabling the non-contact tissue ablations.

The accomplishment of such objectives would eliminate the disadvantages of the stateof-the-art systems such as direct line-of-sight requirement in free-beam systems, tissue sticking problem in fiber-based systems.

In addition to providing an endoscopic laser scanner for microsurgeries, the objectives include also providing assistive features to the surgeon to control the incision depth in endoscopic soft tissue microsurgeries. State-of-the-art laser systems do not offer assistance to control the laser incision depth. The precision and quality of the incisions are entirely dependent on the surgeons visual perception and experience. Additionally, unexperienced surgeons do not know how to regulate the laser parameters such as power, time, scanning width, number of passes, etc., to achieve desired incision depths. Thus, the aim is to develop a controller which regulates laser parameters automatically given a desired incision depth. 


\subsubsection{Thesis outline}

The work presented in this thesis is focused on development of a magnetically actuated laser scanner for endoscopic applications and on the proof-of-concept of a technology for incision depth control as an assistive feature to surgeons.

The outline of the thesis is as follows (see also Fig. 1-3):

\section{- Chapter 1 - Introduction}

Chapter 1 starts with the general applications of laser scanners. Then, significance of the laser scanners in microsurgery is explained. Current status of laser-based systems for microsurgery is discussed. Then, advantages of the endoscopic laser control systems are highlighted for minimally invasive surgeries.

\section{- Chapter 2- Magnetic Laser Scanner Design}

Chapter 2 introduces proposed magnetic laser scanner and gives the details of the design. Additionally, design constraints are discussed for an endoscopic tool with magnetic actuation. Details of the experimental setup are demonstrated with magnetic laser scanner, laser source, camera, tablet device for teleoperation user trials.

\section{- Chapter 3 - Characterization Experiments}

In this chapter, characterization experiments of the magnetic laser scanner are presented with workspace, step and frequency responses and repeatability.

\section{- Chapter 4-Modeling, Identification and Feed-Forward Control}

In this chapter, a dynamic model is presented for magnetic laser scanner based on the observations from characterization experiments. Model parameters are identified with a set of experiments. Finally, a feed-forward controller is developed and first experiments are presented.

\section{- Chapter 5 - Teleoperation User Trials}

In this chapter, the usability studies of the magnetic laser scanner and accuracy assessment were presented with user trials. Different target trajectories that 


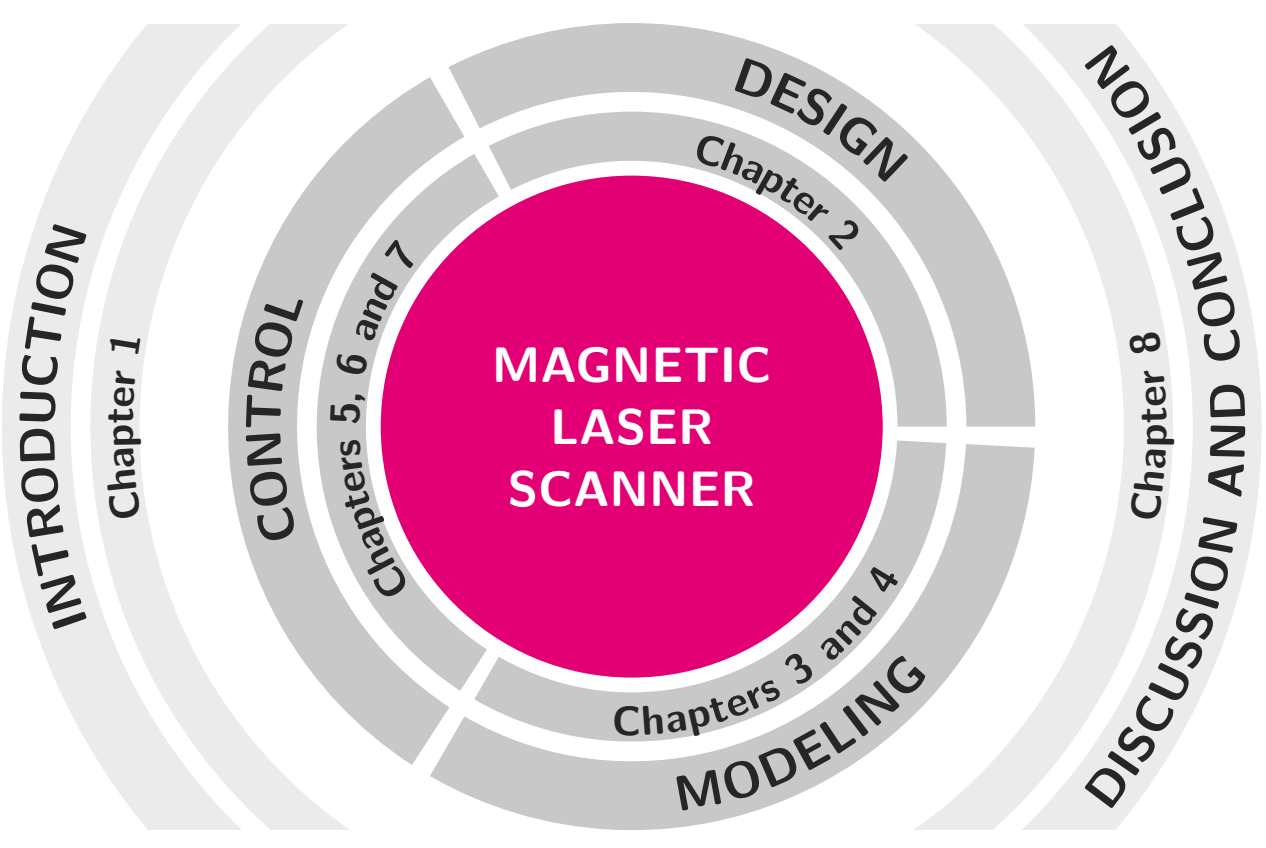

Figure 1-3: Thesis outline.

challenge precise control by the user were defined. Then, subjects executed the trajectories with magnetic laser scanner and a tablet device. A dedicated questionnaire was also performed for subjective analysis.

\section{- Chapter 6-Automated Trajectory Executions}

Surgeons generally excise tissue by performing deep incisions, which are achieved through repeated passes of the laser over the same trajectory. Such task can be achieved automatically by the magnetic laser scanner. To present the accuracy of the scanner, 2D trajectories are executed with feed-forward controller that is presented in Chapter 4. The results are discussed considering typical requirements for minimally invasive surgeries.

\section{- Chapter 7- Laser Incision Depth Control}

State-of-the-art systems do not include any feature for the estimation of the laser incision depths. In this chapter, a proof-of-concept study is presented for controlling the incision depths in soft tissue microsurgeries. First, a model for incision depth is developed including energy density and repetitive passes. 
Then, a controller is proposed with the developed model to achieve target incision depths.

\section{- Chapter 8 - Discussion and Conclusion}

This chapter discusses the contributions of this thesis and offer conclusions related to the proposed technology. In addition, it discusses future directions for improving the achieved results. 



\section{Chapter 2}

\section{Magnetic Laser Scanner Design}

A magnetic laser scanner was designed considering the requirements of the endoscopic microsurgeries discussed in the previous chapter. The proposed system actuates an optical fiber with a compact magnetic actuation mechanism. This chapter starts with the conceptual design of the system. The details of the prototype manufacturing are explained for a proof-of-concept device. Then, the experimental setup and different operating modes are explained. Finally, the evaluation methodology for assessing experimental results is presented.

\subsection{Conceptual Design}

The magnetic laser scanner was designed as an end-effector module for a continuum robot arm. Fig. 2-1 shows the conceptual design of the proposed magnetic laser scanner for endoscopic microsurgery. The main design objective is to provide laser position control at the tip of a flexible robotic arm. The system includes a flexible optical fiber for delivering the high-power surgical laser to the distal end of the tool, a permanent magnet on the optical fiber, four electromagnetic coils, and two optical lenses to focus the laser beam on a target. The basic working principle relies on the fact that the laser spot position changes on the target surface by bending the cantilevered optical fiber. In this design, magnetic actuation is proposed to bend the optical fiber. 


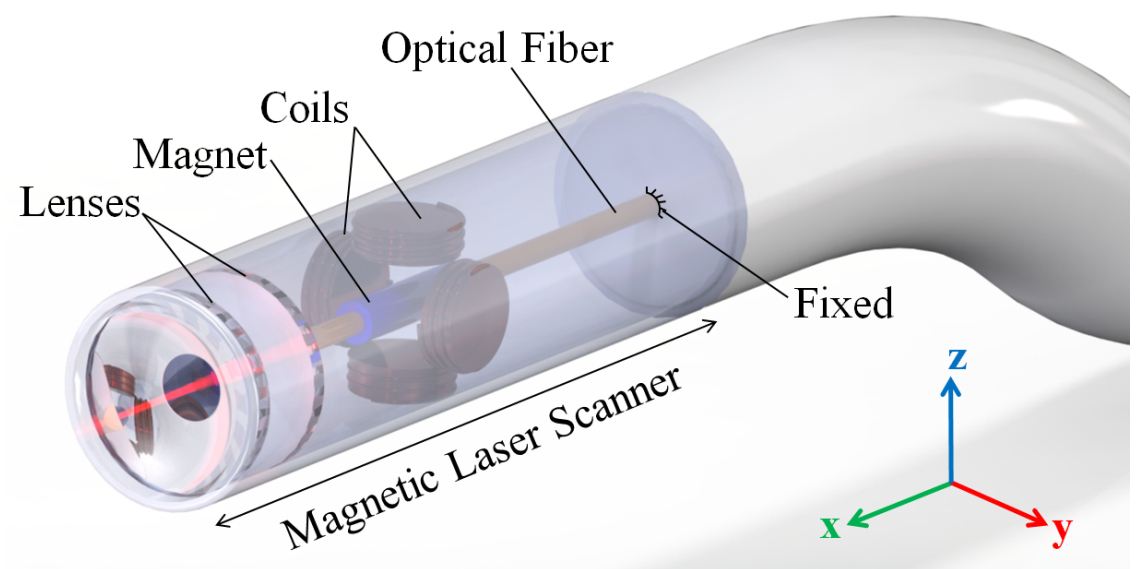

Figure 2-1: Conceptual design of proposed magnetic laser scanner as an end-effector module of a flexible robotic arm. The magnetic laser scanner consists of an optical fiber, electromagnetic coils, a permanent magnet and optical lenses. The micromanipulation of the optical fiber is based on magnetic interaction between electromagnetic coils and the permanent magnet.

\section{$2.2 \quad$ Prototype Manufacturing}

The mechanical design of the device is based on the optimization of different constraints. The main constraint is the overall diameter of the magnetic laser scanner. The second constraint is the requirement of maximum magnetic field strength to bend the cantilevered optical fiber so as to increase the total workspace of the tool: maximum number of turns must be wound on the electromagnetic coils to create maximum torque on the permanent magnet. In addition to these, four electromagnetic coils are placed for 2D position control of the laser spot. Thus, the optimization of the constraints can be defined as: placing four electromagnetic coils with maximum possible sizes inside a cylindrical tool with minimum outer diameter. In the current proof-of-concept design, the overall diameter of the tool is restricted to be less than $15 \mathrm{~mm}[44,45]$.

The proof-of-concept device was manufactured as follows: The cylindrical base of the electromagnetic coils, lens and fiber holders were manufactured with 3D printing (see Fig. 2-2). Four ferromagnetic coil cores were placed circumferentially on this cylindrical base orthogonal to each other. Then, electromagnetic coils were wound on the cores with a thin copper wire. Optical fiber was fixed to a fiber holder. An 
Table 2.1: Prototype Components of Magnetic Laser Scanner

\begin{tabular}{lc}
\hline \hline Component Name & Specification \\
\hline Visible laser source & Visible red, $\lambda=625 \mathrm{~nm}$ \\
Surgical diode laser & $\lambda=1940 \mathrm{~nm}$ \\
Multimode optical fiber & $\varnothing_{\text {core }}=300 \mu \mathrm{m}, \mathrm{NA}=0.39$ \\
Collimating lens & $f=12.5 \mathrm{~mm}, \varnothing=9 \mathrm{~mm}$ \\
Focusing lens & $f=30 \mathrm{~mm}, \varnothing=6 \mathrm{~mm}$ \\
Ring permanent magnet & $\varnothing_{i}=0.7 \mathrm{~mm}, \varnothing_{o}=2 \mathrm{~mm}, h=2 \mathrm{~mm}$ \\
Electromagnetic coils & 150 turns, iron core \\
\hline
\end{tabular}

a)

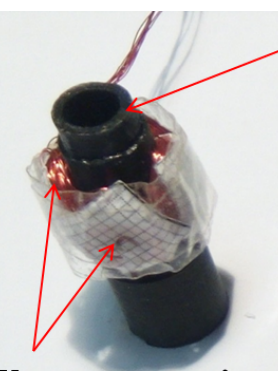

Electromagnetic

Coils

\section{Cylindrical Base}
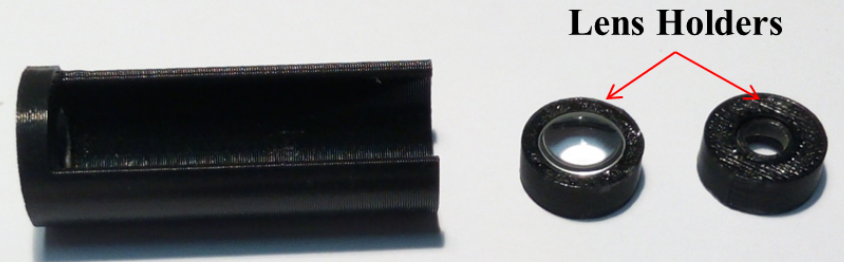

Fiber

Holder

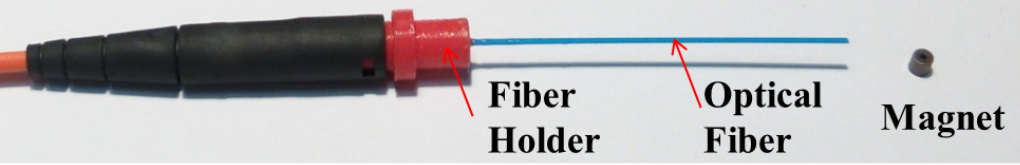

b)

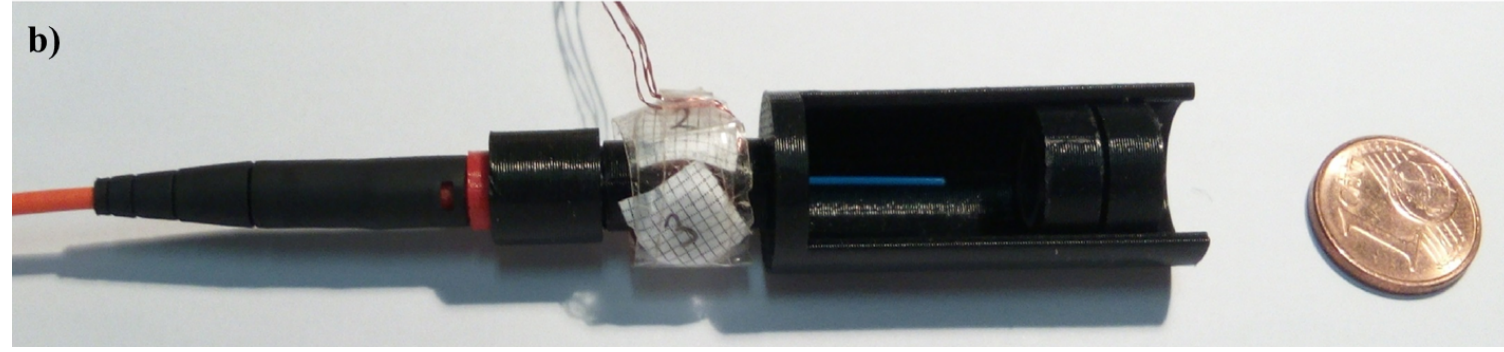

Figure 2-2: Prototype components (a) and assembled prototype (b) of magnetic laser scanner. 
axially magnetized ring permanent magnet was inserted over the optical fiber. Lens holders were attached to the cylindrical base which holds the electromagnetic coils. Table 2.1 presents component specifications of the magnetic laser scanner prototype. The optical lenses were chosen to keep a $30 \mathrm{~mm}$ distance between the tip of the magnetic laser scanner and the target surface. This optical system produced a laser spot diameter of $0.57 \mathrm{~mm}$ on the target (focal length $=30 \mathrm{~mm}$ ). The total scanning range achieved with this design is $4 \times 4 \mathrm{~mm}^{2}$.

\subsection{Experimental Setup}

The experimental setup developed to model and control the magnetic laser scanner is presented in Fig. 2-3. The optical fiber has a $300 \mu \mathrm{m}$ diameter core and 0.39 NA (Thorlabs FT300EMT). A ring permanent magnet is inserted over the optical fiber. The sizes of the permanent magnet are $2 \mathrm{~mm}$ outer diameter, $0.7 \mathrm{~mm}$ inner diameter and $2 \mathrm{~mm}$ height. Each electromagnetic coil has 150 turns of a copper magnet wire with a ferromagnetic cylindrical core. The length of the cantilevered optical fiber is $37 \mathrm{~mm}$. Two plano-convex lenses with, respectively, $12 \mathrm{~mm}$ and $30 \mathrm{~mm}$ focal lengths are used to collimate and focus the laser beam on the target (Thorlabs LA1576 and LA1700). The focusing lens defines the working distance between the tool and the target.

The prototype has $13 \mathrm{~mm}$ overall diameter and $60 \mathrm{~mm}$ length with 3D printed cylindrical structures that hold the electromagnets. The overall diameter is primarily limited by the surgical requirements. However, this is restricted by the need to place four electromagnetic coils with a maximum number of turns to increase the magnetic field strength. Thus, it is a design constraint placing largest electromagnets in a cylindric component with minimum overall diameter.

The wire that is used to create electromagnetic coils defines the maximum current. The electromagnetic coils are wound with 36 AWG copper wire with $0.15 \mathrm{~mm}$ diameter. The maximum current that is fed to the electromagnetic coils is $165 \mathrm{~mA}$ 


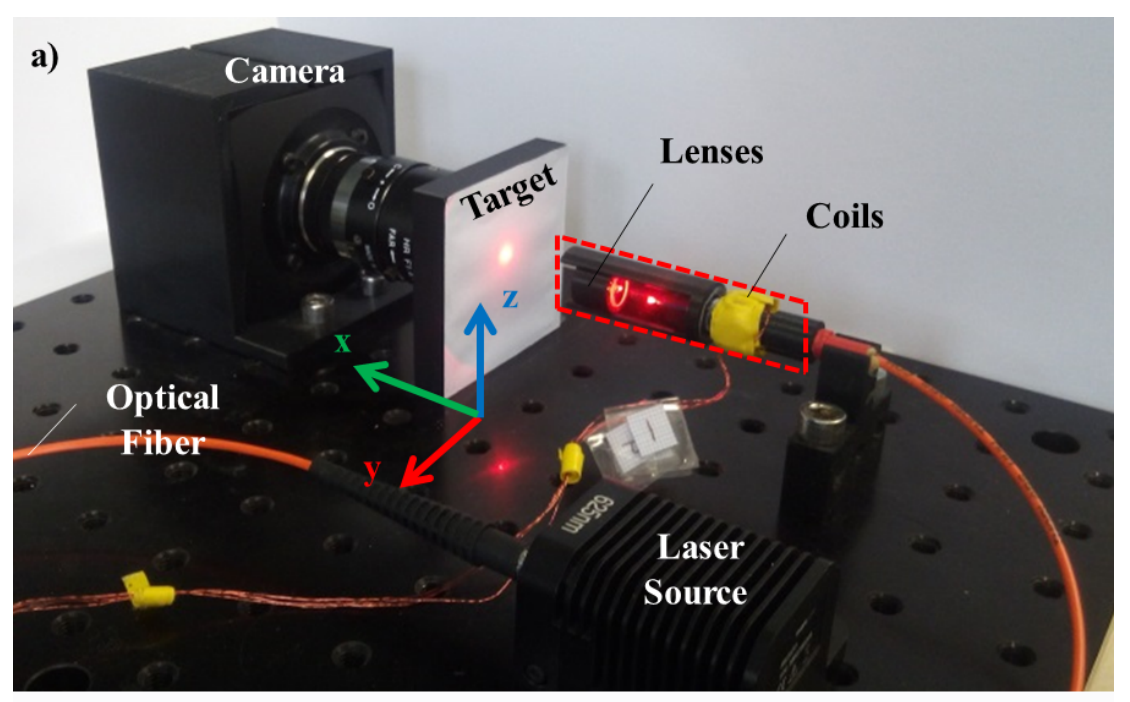

b)

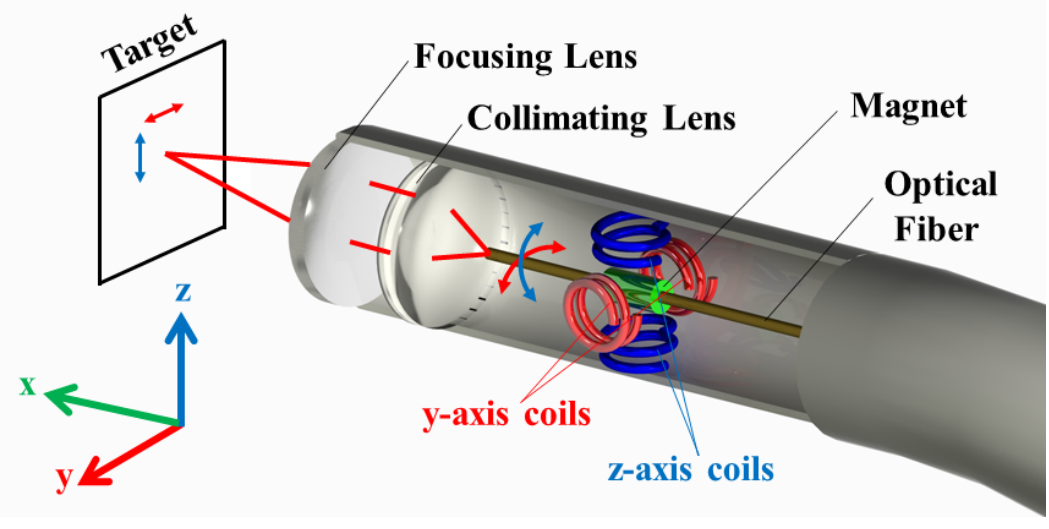

Figure 2-3: a) Experimental setup including the magnetic laser scanner, optical fiber, lenses, laser source and camera. b) Presentation of collimation and focusing of laser beam with two optical plano-convex lenses.

and the applied voltage is limited to $9 \mathrm{~V}$. Low actuation voltage and currents are important for the safety of the surgical procedures. For this reason, maximum values are restricted to the specified values.

An Arduino Due microcontroller and custom current control circuits are used to control the coil pair currents independently. The microcontroller is connected to a computer via USB and is capable of receiving real-time commands at a rate of 4 $\mathrm{KHz}$ [73]. Its two 12-bit digital to analog converters (DAC) are used to drive the current control circuits, and thus control the currents flowing through the y- and z-axes coils. 


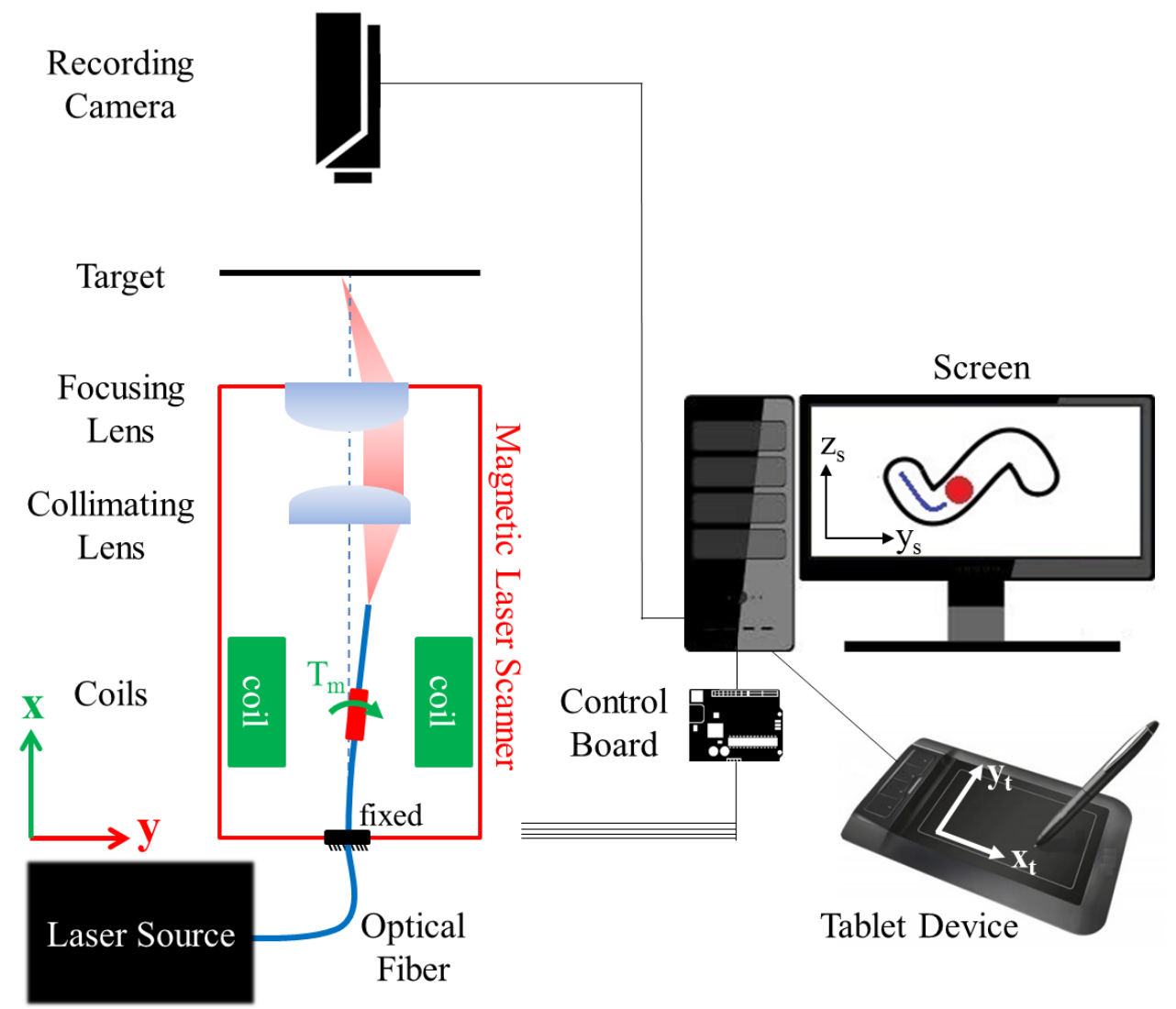

Figure 2-4: Schematic illustration of the experimental setup. For simplicity, one degree of freedom is presented for magnetic actuation in the x-axis. Laser spot position control on the computer screen with a tablet device is shown on the right side of the figure.

\subsection{Teleoperation Control Setup}

Fig. 2-4 shows the experimental setup for teleoperation user trials. The control board and a tablet device are connected to a computer via USB.

These devices are interconnected by using Robot Operating System (ROS) to control the current levels $I_{y}$ and $I_{z}$, with the tablet positions $x_{t}$ and $y_{t}$, respectively. This allows to map the tablet coordinates to the currents, $I_{y}$ and $I_{z}$ :

$$
\left[\begin{array}{l}
I_{y} \\
I_{z}
\end{array}\right]=\left[\begin{array}{ll}
c_{11} & c_{12} \\
c_{21} & c_{22}
\end{array}\right]\left[\begin{array}{l}
x_{t} \\
y_{t}
\end{array}\right],
$$

where $c_{11}, c_{12}, c_{21}$ and $c_{22}$ are four scalars. 
The tablet is a mouse-like device that provides absolute position coordinates. Its position values change between -1 and +1 in the $\mathrm{x}$ - and $\mathrm{y}$-axes. The current control board provides 4096 levels for each current $\left(I_{y}\right.$ and $\left.I_{z}\right)$. The mapping matrix scales the tablet positions $[-1,1]$ to the current control levels [-2047, 2048].

In the experimental setup, reference coordinate systems for magnetic laser scanner $(y, z)$, screen $\left(y_{s}, z_{s}\right)$ and tablet $\left(x_{t}, y_{t}\right)$ are aligned to each other. This alignment implies that tablet x-position deflections affect only $I_{y}$ and tablet y-position deflections affect only $I_{z}$. In other words, coefficients $c_{12}$ and $c_{21}$ equal to $0 . c_{11}$ and $c_{22}$ are equal, i.e., c. In this experimental setup, $c$ value is defined as 2047.

$$
\left[\begin{array}{l}
I_{y} \\
I_{z}
\end{array}\right]=\left[\begin{array}{ll}
c & 0 \\
0 & c
\end{array}\right]\left[\begin{array}{l}
x_{t} \\
y_{t}
\end{array}\right] .
$$

If the frames are not aligned, same control method can be implemented with an additional calibration step. A calibration would be done by recording a set of tablet positions with the corresponding current values. Recorded values can be used to calculate coefficients $c_{11}, c_{12}, c_{21}$ and $c_{22}$.

\subsection{Operating Modes}

The magnetic laser scanner provides three operating modes, incorporating the features of state-of-the-art systems and offering an additional assistive feature for improved surgical experience:

- High-speed scanning: In this mode, the system actuates the laser to perform fast and continuous motions for improved quality of cuts. Characterization of high-speed scanning is performed with frequency analysis in Chapter 3.

- Intra-operative incision planning: During surgeries, surgeons generally define an incision path and execute this trajectory with repetitive manual passes. During these repetitions, keeping the laser spot exactly on the same path is highly important to avoid unexpected incisions in the vicinity of the desired path. The 


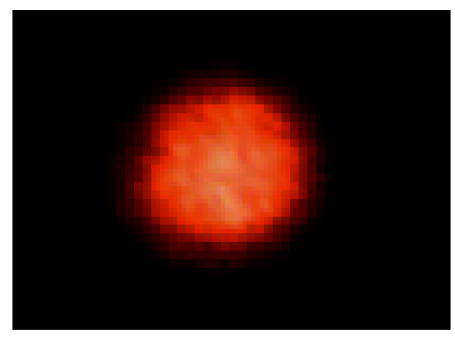

a) Original Frame

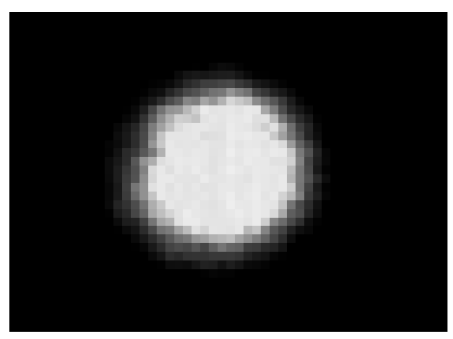

b) Red Channel

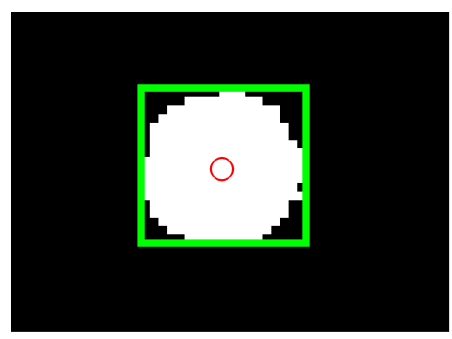

c) Detected Laser Spot

Figure 2-5: Detection of laser spot in three steps: capturing the current frame (a), presenting the frame in red channel (b) and detection of the laser spot (c). Green rectangle presents the bounding rectangle of the laser spot. Red circle presents the centroid of the detected region.

magnetic laser scanner allows surgeons to create incision paths intra-operatively and then the system can perform automatic repeated incisions on the same path. Characterization of this mode is presented with the repeatability experiments described in Chapter 3.

- $2 D$ position control: In this mode, the laser spot position is controlled in the total workspace with free-hand motions on the tablet device. Characterization of this tablet control is done through teleoperation user trials described in Chapter 5.

\subsection{Evaluation Methodology}

\subsubsection{Laser position detection}

In order to extract the laser positions for the characterization experiments, videos are recorded with a high-speed camera (SpeedCam HiSpec 1 - Fastec Imaging) which is able to capture images at $1000 \mathrm{fps}$. For the teleoperation user trials, the executed trajectories with a tablet device are recorded with a Prosilica GT1910C (Allied Vision) camera at $25 \mathrm{fps}$ as illustrated in Fig. 2-4. The recorded color images are postprocessed with MATLAB image processing tools in order to get the laser spot position in each frame.

The implemented laser detection algorithm processes the red channel of the image 
(since the laser used was red) and is based on the intensity values of the pixels. Locations of pixel group with intensity values above a certain threshold are recorded. Then, the centroid of the pixel group is accepted as the location of the laser spot (Fig. 2-5). The threshold value is calibrated manually. It is defined between 0 and 1 depending on the illumination of the experimental setup. This algorithm allows to define the location of centroid with sub-pixel resolution.

Pixel dimensions of the recorded images are measured using a piece of millimetric paper as target surface: 62 pixels $\cong 1 \mathrm{~mm}$. Thus, the measurements in pixel units are converted to $\mathrm{mm}$ with a scale factor of $16.1 \mu \mathrm{m} /$ pixel. Separately, it is ensured that the entire region of the target trajectory is in focus and fully within the camera image plane.

For the workspace assessment, the initial laser position, when no current is flowing through the electromagnet coils, is accepted as the reference point $\left(\left[I_{y}, I_{z}\right]=[0,0]\right.$ $\left.\longrightarrow\left[d_{x}, d_{y}\right]=[0,0]\right)$. Then, as the currents are changed, new laser positions are measured with respect to that reference point.

\subsubsection{Trajectory errors}

For the assessment of the executed trajectories, the errors are calculated by measuring the closest distance to the target trajectory for each measurement point. For the $i^{\text {th }}$ measurement point, the error is:

$$
\operatorname{err}(i)=\min _{\forall k}\left(\left|d_{m}(i)-d_{t}(k)\right|\right)
$$

where $d_{m}$ is the executed trajectory and $d_{t}$ is the target trajectory (Fig. 2-6). Maximum error is the highest value of err, and root-mean-square-error (RMSE) is calculated as:

$$
\mathrm{RMSE}=\sqrt{\frac{1}{N_{t}} \sum_{i=1}^{N_{t}} \operatorname{err}(i)^{2}}
$$

where $N_{t}$ is the total number of measurement points.

For the assessment of the characterization experiments, the root-mean-square er- 


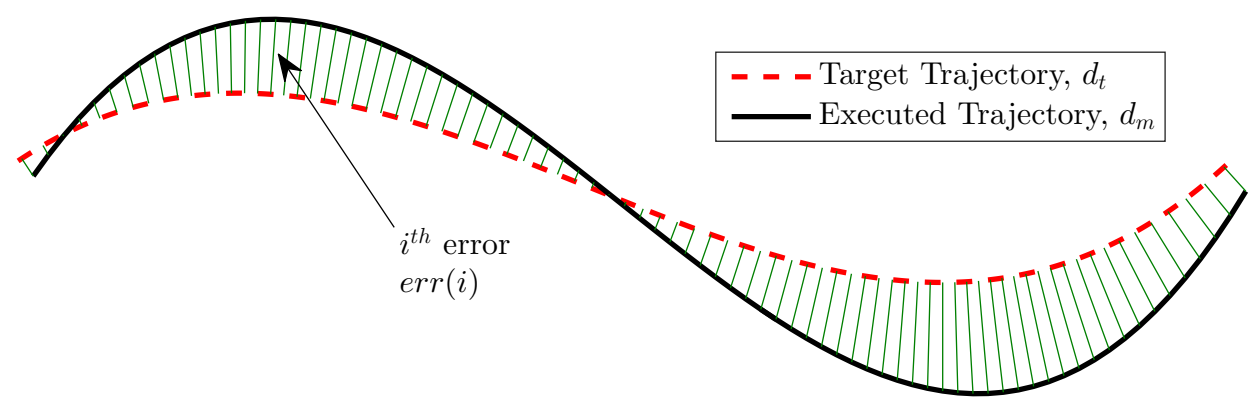

Figure 2-6: Presentation of the trajectory error measurement with the target and executed trajectories.

rors (RMSE) are calculated based on the reference trajectories. For the assessment of the teleoperation user trials, three metrics are used: (i) the RMSE based on the target trajectories, (ii) the maximum errors, and (iii) the execution times. The execution time is extracted from the recorded videos by measuring the time elapsed between the start of the execution of a trajectory until its completion. For RMSE and maximum errors, the executed trajectories are compared with the target ones.

The extent of the workspace of the magnetic laser scanner depends on the optical design. The distance between tip of the tool and the target is defined with the focal length of the focusing lens. Total workspace dimensions change linearly with the focal length. Thus, depending on the working distance between the focusing lens and the target, laser displacement on the target changes for same bending angle of the cantilever beam. In order to present generalizable results for different optical designs, laser spot displacements are converted to bending angles of cantilevered optical fiber. For 1 pixel laser spot deflection, beam bending is measured as $\sim 0.26$ mrad. 


\section{Chapter 3}

\section{Characterization Experiments}

After designing and manufacturing the magnetic laser scanner, characterization experiments were performed to assess the extent of the workspace, repeatability, step and frequency responses.

\subsection{Workspace}

The aim of this experiment was to define the maximum limits of the workspace of the magnetic laser scanner. To this end, a set of current values, $I_{y}$ and $I_{z}$, were fed to the coil pairs. Then, the laser spot positions in the yz-plane and the position $(d)$ current $(I)$ correlations were calculated. Fig. 3-1 presents the 24 different laser spot positions for current values varying between $\pm 0.165 \mathrm{~A}$ in $\mathrm{y}$ - and z-axes.

The position of the laser spot changes linearly with the current (Fig. 3-2). The current values resulted in a workspace almost $4 \times 4 \mathrm{~mm}^{2}$ in size.

In Fig. 3-1, it can be seen that laser spot positions are not perfectly aligned with each other in the workspace. These errors can be explained as resulting from manufacturing imperfections of the magnetic laser scanner. In the analysis, it is assumed that four identical electromagnetic coils are used. However, independent impedance measurements have shown that there are 3-5\% differences among them. Another assumption is that the coils facing each other on the same axis are placed

perfectly parallel to each other. However, the misalignment of the coil pairs in x- and 


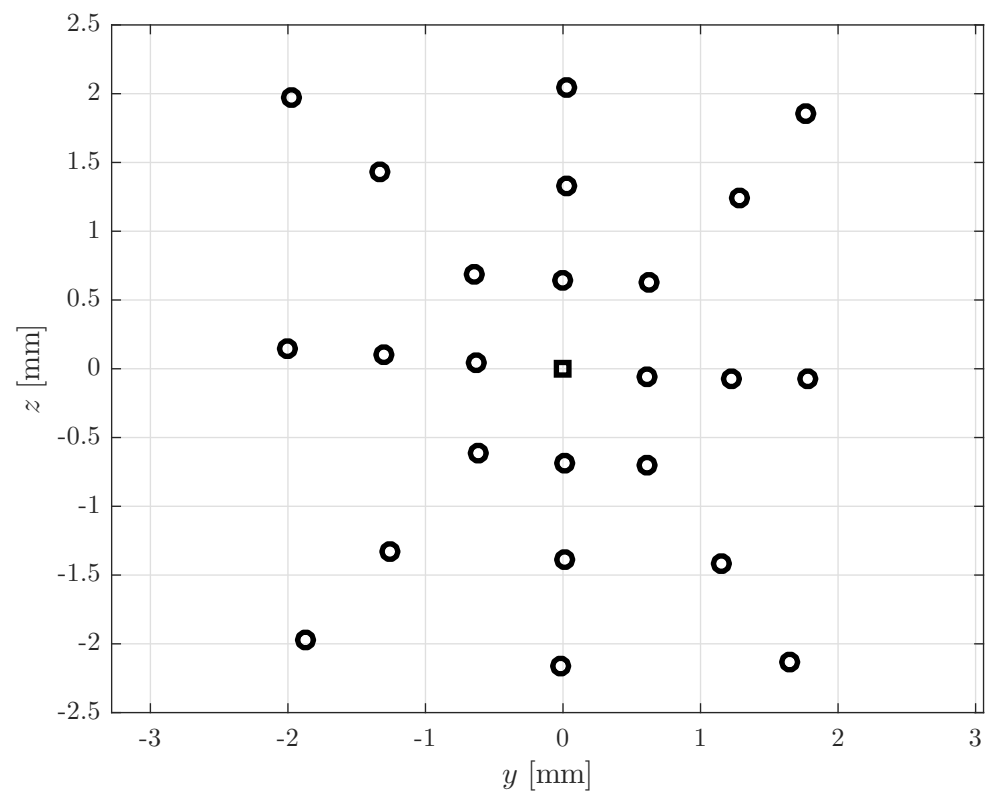

Figure 3-1: Laser spot positions for different current values on the target surface. Black square in $[0,0]$ represents the reference point, i.e., the initial laser position on the target for currents $I_{y}=0$ and $I_{z}=0$.
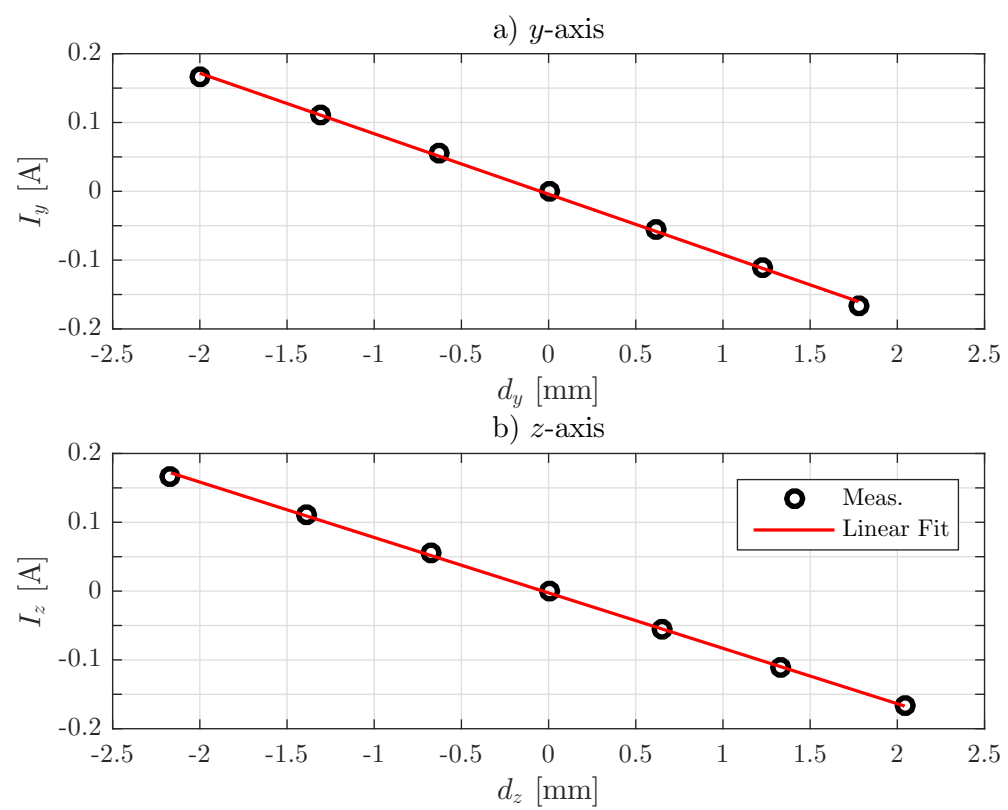

Figure 3-2: Current $(I)$ - displacement $(d)$ plots for y-axis (a) and z-axis (b). 
y-axes can cause different deflections on the positive and negative sides of the axes. These problems can possibly be eliminated with better manufacturing and assembly of the device. Alternatively, system identification and modeling can be performed for accurate laser positioning.

\subsection{Repeatability}

Repeatability is an important criteria for laser microsurgery. During computercontrolled repetitions of predefined intra-operative trajectories, the laser spot should travel exactly on the same path in order to guarantee accurate operation. This requirement becomes important when surgeons want to perform repeated ablations along customized paths. In order to measure the repeatability of trajectories, a $2 \mathrm{D}$ trajectory with an '8' figure was executed at $1 \mathrm{~Hz}$ and repeated 10 times. In this case, the first pass is accepted as the reference trajectory, allowing the computation of the RMSE for the subsequent passes of the laser spot.

Fig. 3-3 depicts the results from the repeatability assessment considering the ' 8 '

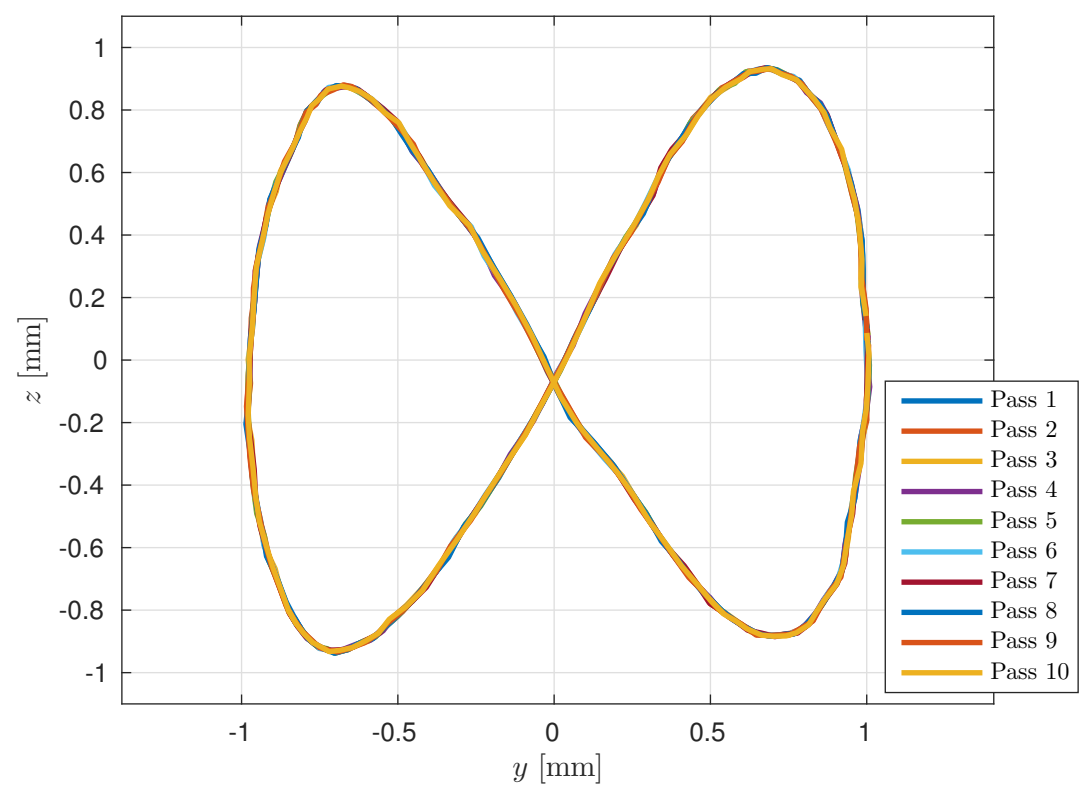

Figure 3-3: Repeatability results of the scan trajectories for 10 passes. All trajectories are overlapping and indistinguishable from each other. 

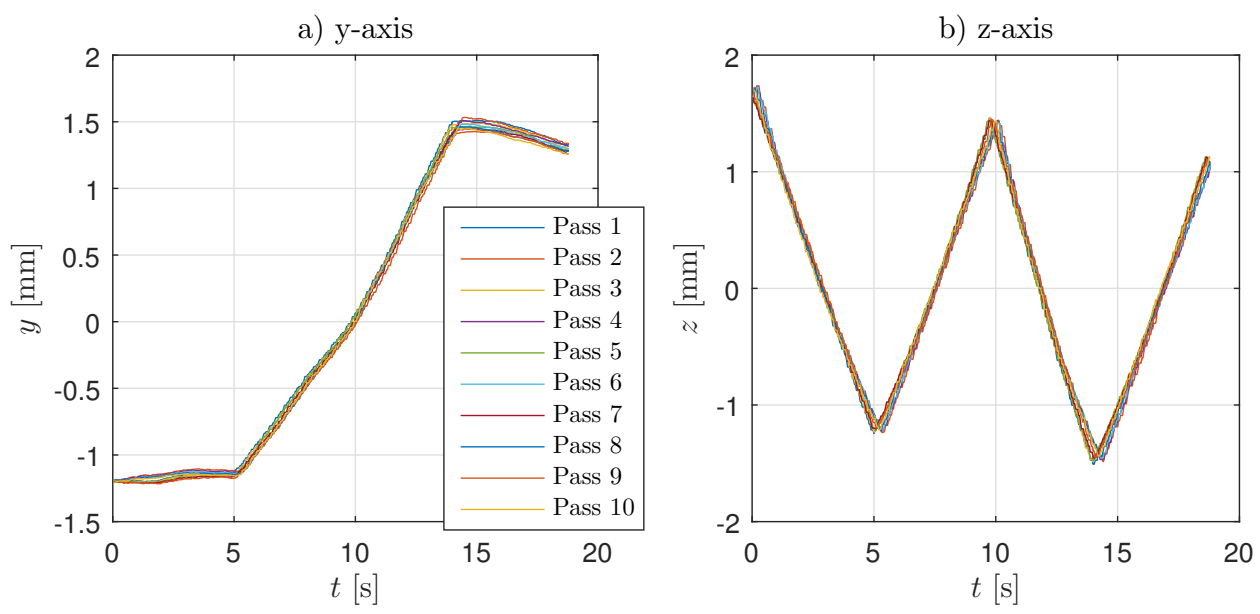

Figure 3-4: Repeatability results of ' $\mathrm{M}$ ' shaped trajectory for 10 passes in $y$-axis (a) and z-axis (b).

figure (number of passes, $n=10$ ). The calculated average RMSE for repeatability trials based on the first pass is $21 \pm 10 \mu \mathrm{m}$. Thus, the system shows very high repeatability and is, therefore, suitable for precise intra-operative incisions during surgery.

In addition to ' 8 ' figure, an 'M' shaped trajectory was also chosen because it is challenging for fast scanning. Similarly to the previous case, the same trajectory was repeated 10 times (number of passes, $n=10$ ). The first pass was accepted as the reference trajectory, allowing the computation of the root mean square error (RMSE) for the subsequent passes of the laser spot over the desired trajectory.

Results from the repeatability assessment considering the ' $\mathrm{M}$ ' shaped trajectory were presented in Fig. 3-4 for the y- and z-axes trajectories. The RMSE calculated for each axis resulted in: $\mathrm{RMSE}_{y}=48 \pm 16 \mu \mathrm{m}$ and $\mathrm{RMSE}_{z}=75 \pm 35 \mu \mathrm{m}$.

\subsection{Step Response}

The step response of the magnetic laser scanner has been observed by switching on/off the system with a current value $0.165 \mathrm{~A}$ and by measuring the laser spot positions. In Fig. 3-5 presents the typical step response of magnetic laser scanner. The final value of the actual response is $\sim 1.8 \mathrm{~mm}$. The time required for the response increase 


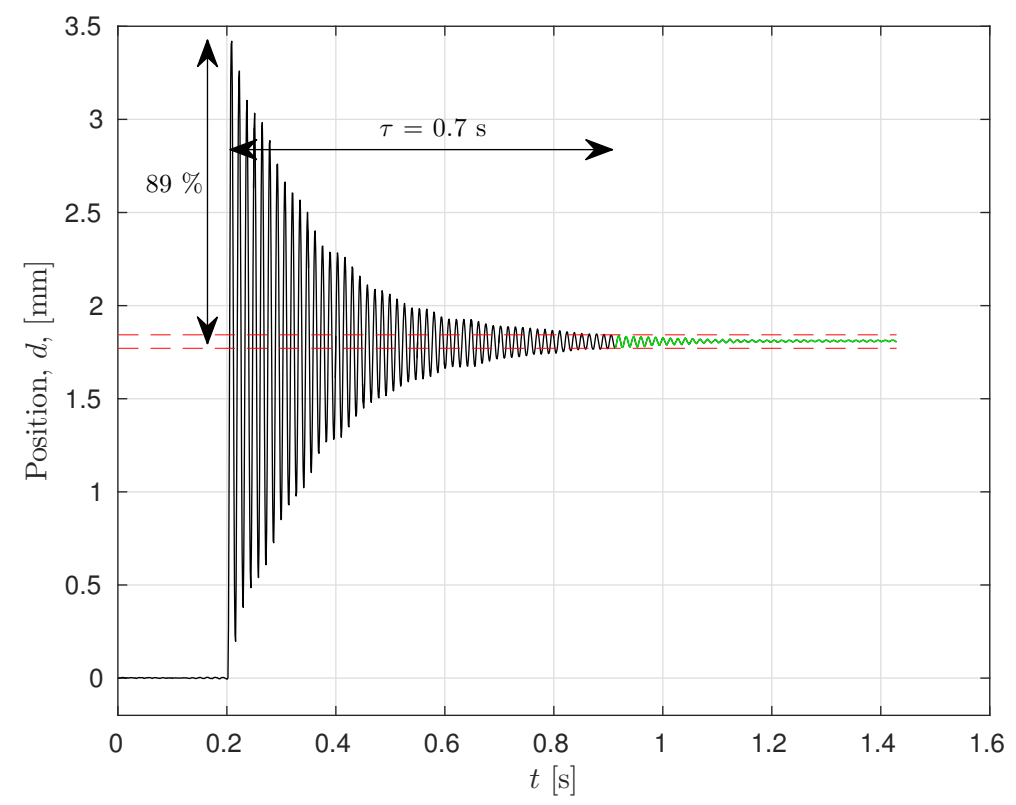

Figure 3-5: Typical step response of the laser spot deflection for a current value of 0.165 A with settling time $\tau=0.7 \mathrm{~s}$, and overshoot percentage $P O=89 \%$. Dashed red lines indicate the $2 \%$ range for the settling criterion. Settled response is highlighted with a green line.

from $10 \%$ to $90 \%$ of its final value, rise time $T_{r}$ is measured as $0.002 \mathrm{~s}$. Settling time is measured as $\tau=0.7 \mathrm{~s}$, when the response is in the range of $2 \%$ of the final value. Overshoot percentage, $P O$, is calculated as $89 \%$ with respect to the final value.

\subsection{Frequency Response}

As mentioned in Chapter 1, the fast motion of the laser spot is crucial for clean incisions and minimal thermal damage to the surrounding healthy tissue. In order to characterize the high-speed scanning feature, experiments were conducted by scanning a $0.72 \mathrm{~mm}$ straight line with different excitation frequencies up to $100 \mathrm{~Hz}$. Current values are calculated with sinusoidal functions

$$
I=A \sin (2 \pi f t)
$$

where $A$ is current amplitude, $f$ is excitation frequency and $t$ is time. 


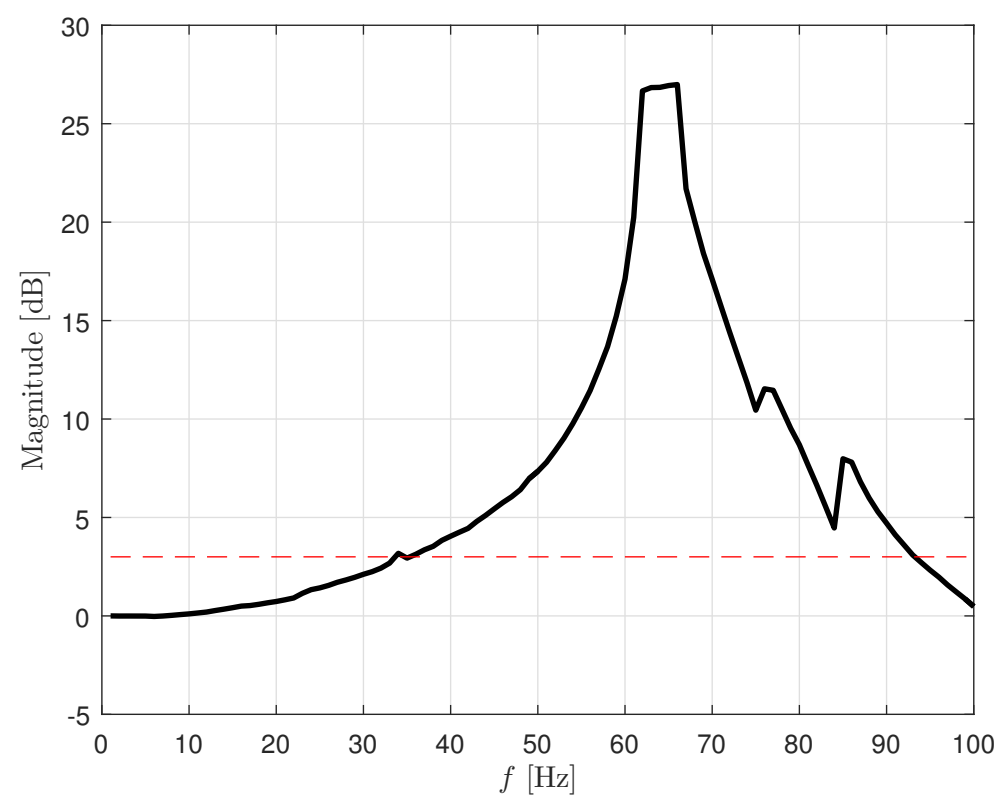

Figure 3-6: Frequency response of the magnetic laser scanner. Magnitude $[\mathrm{dB}]$ excitation frequency $[\mathrm{Hz}]$ graph is presented. Red dashed-line shows $3 \mathrm{~dB}$ limit for cut-off frequency.

Fig. 3-6 depicts the magnitudes for different excitation frequencies. The magnitude increases as excitation frequency is increased. For $3 \mathrm{~dB}$ limit, cut-off frequency is $33.6 \mathrm{~Hz}$. As excitation frequency gets closer to the natural frequency of the system, the magnitude of the bending angle increases exponentially as expected. The observed natural frequency, $f_{n}$, is $63 \mathrm{~Hz}$ and its corresponding circular natural frequency, $\omega_{n}=2 \pi f_{n}$, is $395.8 \mathrm{rad} / \mathrm{s}$. When the system is operated at the natural frequency, bending of the fiber reaches the physical limits of the tool; i.e., the fiber touches the inner wall of the cylindrical tool which holds the electromagnets.

The length of the line was chosen as $0.72 \mathrm{~mm}$ to be able to collect data from each excitation frequency experiments. During the scanning of longer lines, the optical fiber touches the inner wall at lower frequencies than the natural frequency. This prevented to define the correct natural frequency of the system. For that reason, a small scanning line was scanned for characterization. 


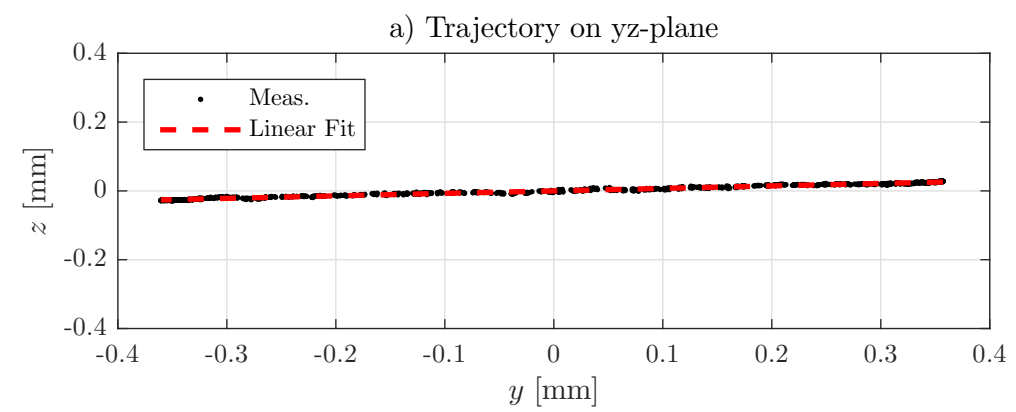

b) Time - Position

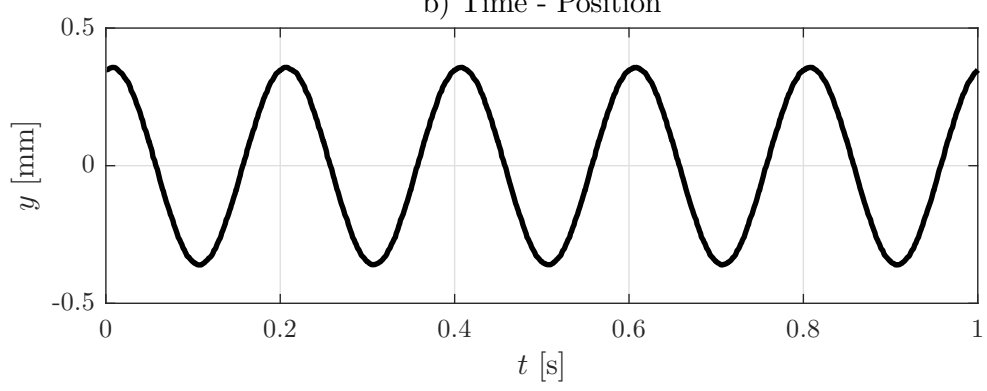

Figure 3-7: a) A sample trajectory for RMSE calculation to demonstrate the deviation from linearity. b) Time-position graph for the sample trajectory.

\subsection{Deviation from linearity}

During high-speed scanning of a straight line, deviation from linearity is an important parameter for scanning laser systems. In order to observe the deviation from this linear path, a linear fit to the measurement points is accepted as reference. Then RMSE values are calculated based on this reference for all excitation frequencies.

In Fig. 3-7a and b, a sample trajectory is shown for $5 \mathrm{~Hz}$ for the comparison of the measurement points to the linear fit. The same method is repeated for all excitation frequencies and RMSE values are presented in Fig. 3-8. RMSE values increase exponentially as the excitation frequency is increased up to the natural frequency of the system. Considering the thermal damage width values (about $50 \mu \mathrm{m}$ ) reported in previous research [24], the maximum stable scanning speed is chosen at $48 \mathrm{~Hz}$ for the errors less than $50 \mu \mathrm{m}$ (Fig. 3-8). 


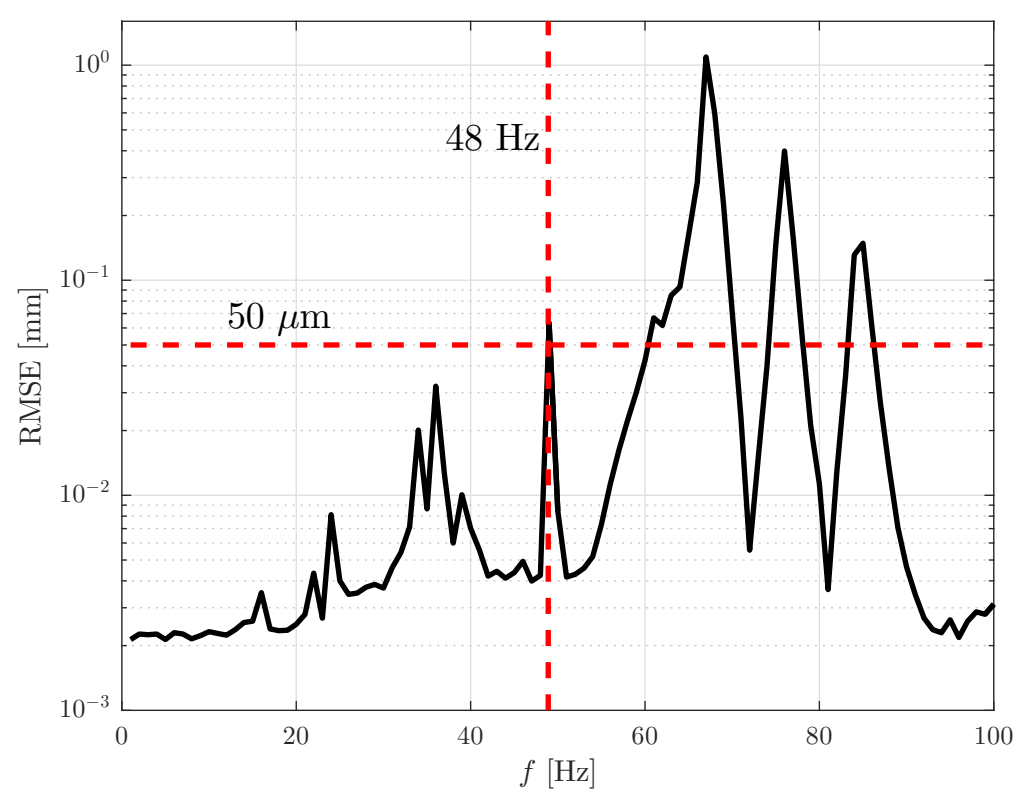

Figure 3-8: Calculated RMSE for different excitation frequencies up to $100 \mathrm{~Hz}$. Average speed of the laser spot on the target was 1.44 and $144.2 \mathrm{~mm} / \mathrm{s}$ for 1 and $48 \mathrm{~Hz}$, respectively.

\subsection{Preliminary Ablations Experiments}

Fig. 3-9 presents the experimental setup for ablation experiments. The magnetic laser scanner was coupled with a $1940 \mathrm{~nm}$ diode laser (Lumics, LuOcean P2) to demonstrate the ablation of different structures. The laser was connected to magnetic laser scanner using a $300 \mu \mathrm{m}$ core multimode optical fiber (ThorLabs, FT300EMT). Ablations were performed on the plaster blocks and apple samples for the preliminary evaluation of the system. Apple samples were chosen due to the high water content that makes them suitable target for diode lasers. In order to measure the ablation parameters, confocal images collected with a Leica SP5 microscope were processed with an extended depth of field algorithm [74]. Ablation depths were calculated by measuring and averaging the depth of 20 different cross sections in the central region of the ablations. Cross sections were selected with $20 \mu \mathrm{m}$ intervals.

The actuation of the magnetic laser scanner was performed by providing the same current values to the coils in the same axis. The current values are presented as $I_{y}$ and $I_{z}$ for the coil pairs in y- and z-axes, respectively. Sinusoidal current profiles were 


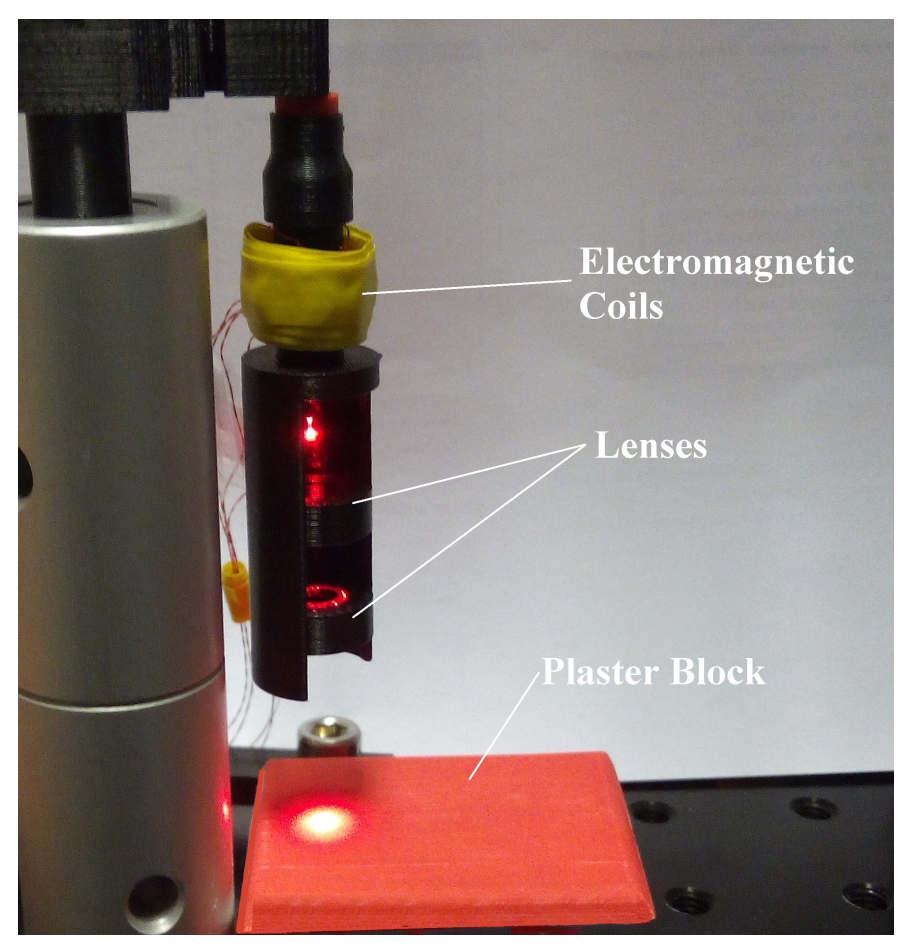

Figure 3-9: Experimental setup with the magnetic laser scanner and plaster block as a target.

fed to the coils

$$
\begin{aligned}
& I_{y}=I_{\text {max }} \sin \left(2 \pi f t_{1}\right), \\
& I_{z}=I_{\text {max }} \sin \left(2 \pi f t_{2}+\phi\right),
\end{aligned}
$$

where $I_{\max }$ is the maximum current, $f$ is the frequency and $\phi$ is phase angle. Different trajectories can be executed by changing the time scale $\left(t_{1} / t_{2}\right)$ and phase angle, $\phi$.

In Fig. 3-10, the results of plaster block ablations are presented. Scan trajectories, in the shapes of 'W', 'Z', '8', and 'I', were executed on the plaster block. Sizes of the trajectories were $2 \times 2 \mathrm{~mm}^{2}$.

Fig. 3-11 shows the depth map of an ablation executed on the apple sample. For these samples, trials were performed using straight trajectories, laser power set to 2 W, and 5 passes (repetitions). Average ablation depth for the sample was $1130 \mu \mathrm{m}$.

Results show that ablations can be performed on plaster and apple samples with 


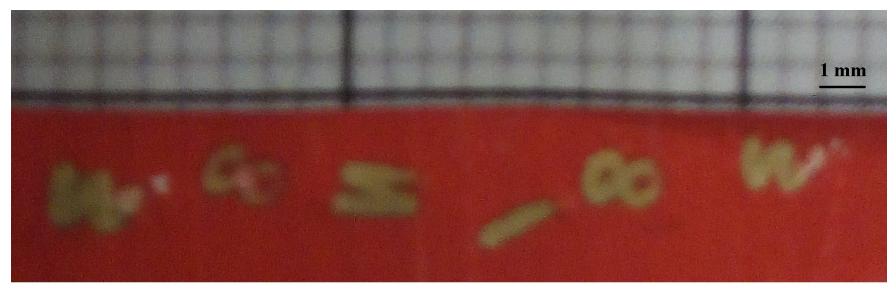

\section{$\mathrm{W} \infty \boldsymbol{S}>\infty \mathrm{W}$}

Figure 3-10: Controlled ablations performed on plaster with $1940 \mathrm{~nm}$ diode laser. The different shapes show that any scan trajectory can be ablated with the magnetic laser scanner.

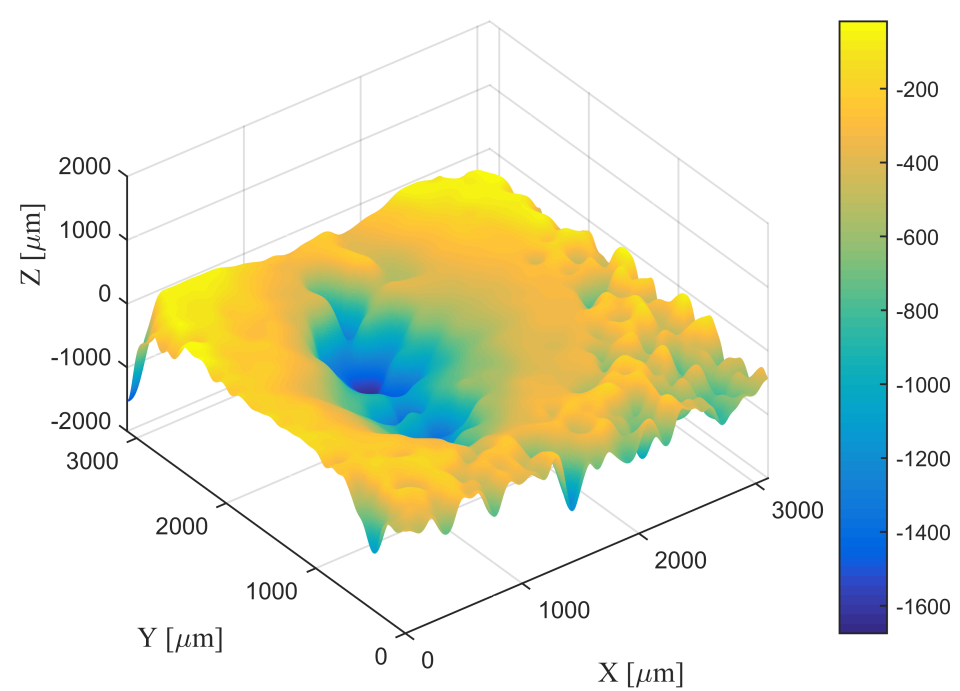

Figure 3-11: Typical 3D ablation profile produced on apple samples by the magnetic laser scanning tool when using the $1940 \mathrm{~nm}$ diode laser set to $2 \mathrm{~W}$ and 5 passes. Colorbar unit is in $\mu \mathrm{m}$.

$1940 \mathrm{~nm}$ diode laser and the proposed magnetic laser scanner. Ablations up to 1.5 mm deep could be executed on the apple sample, showing the system can successfully scan and focus the laser beam at a distant target. 


\section{Chapter 4}

\section{Modeling, Identification and Feed-Forward Control}

\subsection{Modeling}

The magnetic laser scanner is influenced by three main factors: (i) the relation between coil currents and the magnetic torque produced on the permanent magnets, (ii) the mechanical bending of the optical fiber, and (iii) the optical focusing of the laser beam. This chapter focuses on developing a model to represent all these relationships based on experimental observations. The dynamical model of the system is identified by using the electromagnets current (input), the bending angle and the laser position (output). Then, the identified model is used in a feed-forward controller to execute desired trajectories.

\subsubsection{Magnetic actuation}

The principle of the magnetic actuation mechanism is based on the interaction between magnetic field created by coils and a permanent magnet. When current flows through the coils, a magnetic field is induced in the region around coil. 


\section{Single electromagnetic coil}

The magnetic field induced by a circular current loop whose axis lies along z-axis (single loop placed in $z=z_{0}$ plane) (Fig. 4-1) can be expressed in cylindrical coordinate system $\left(r_{c}, \phi, z\right)$ by

$$
\begin{aligned}
B_{r_{c}} & =\frac{\mu_{0} I}{2 \pi} \frac{z-z_{0}}{r_{c}\left[\left(r_{c}+a\right)^{2}+\left(z-z_{0}\right)^{2}\right]^{1 / 2}}\left[-K\left(k_{c}\right)+\frac{r_{c}^{2}+a^{2}+\left(z-z_{0}\right)^{2}}{\left(r_{c}-a\right)^{2}+\left(z-z_{0}\right)^{2}} E\left(k_{c}\right)\right] \\
B_{z} & =\frac{\mu_{0} I}{2 \pi} \frac{1}{\left[\left(r_{c}+a\right)^{2}+\left(z-z_{0}\right)^{2}\right]^{1 / 2}}\left[K\left(k_{c}\right)-\frac{r_{c}^{2}-a^{2}+\left(z-z_{0}\right)^{2}}{\left(r_{c}-a\right)^{2}+\left(z-z_{0}\right)^{2}} E\left(k_{c}\right)\right]
\end{aligned}
$$

where $\mu_{0}$ is the permeability of the free space, $a$ is the radius of the coil, $I$ is the current. $k_{c}^{2}$ is given as,

$$
k_{c}^{2}=\frac{4 a r_{c}}{\left(r_{c}+a\right)^{2}+\left(z-z_{0}\right)^{2}} .
$$

$K\left(k_{c}\right)$ and $E\left(k_{c}\right)$ are the first and the second kind of elliptical integral functions, respectively [75].

The magnetic field components $\left(B_{r_{c}}\right.$ and $\left.B_{z}\right)$ are calculated for any $r_{c}$ and $z$ using (4.1a) and (4.1b). For a point in z-axis $\left(r_{c}=0\right)$, the elliptical integral functions become equal to $\pi / 2(E(0)=\pi / 2$ and $K(0)=\pi / 2)$. Thus, the magnetic field

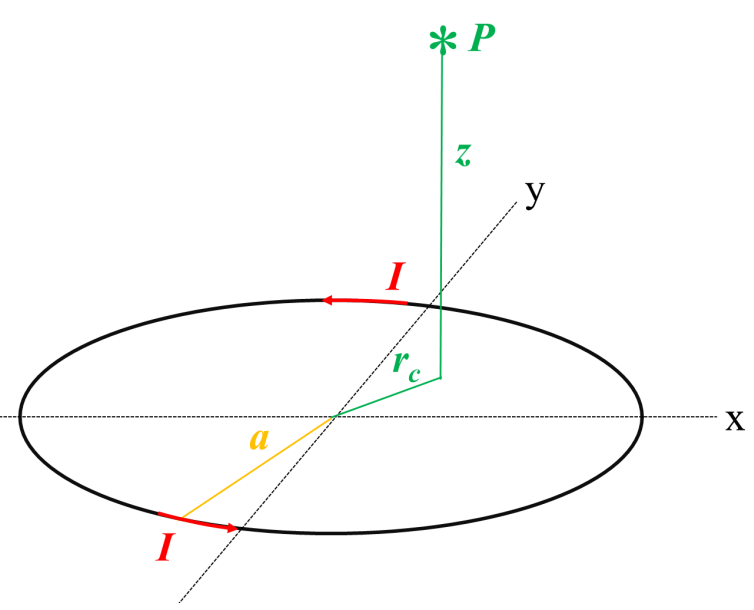

Figure 4-1: Presentation of the point $P$ according to the circular loop which carries current, $I$. 
strength can be derive for the special condition $\left(z_{0}=0\right)[76]$

$$
B_{z}=\frac{\mu_{0} I}{2} \frac{a^{2}}{\left(a^{2}+z^{2}\right)^{\frac{3}{2}}} .
$$

\section{Four electromagnetic coils}

The magnetic laser scanner consists of two coil pairs in y- and z-axes (Fig. 4-2). The magnetic field strengths produced by different coils in the workspace are calculated with (4.1a) and (4.1b). Thus, resultant magnetic field strength, B, can be calculated by vectorial summation of each individual coil contribution.

$$
\mathbf{B}=\sum_{i=1}^{4} \mathbf{B}_{\mathbf{i}}
$$

where $\mathbf{B}$ is a vector composed with $\left[B_{x}, B_{y}, B_{z}\right]$.

\section{Magnetic force and torque}

A permanent magnet in a magnetic field is exposed to a magnetic force and a magnetic

torque. The magnetic force, $\mathbf{F}$, acting on a magnetic dipole moment, $\mathbf{m}$, can be expressed as the gradient of magnetic potential energy, $U$. The magnetic torque, $\mathbf{T}$, is the cross product of the magnetic dipole moment and the magnetic field

$$
\begin{aligned}
& \mathbf{F}=\nabla(\mathbf{m} \cdot \mathbf{B}), \\
& \mathbf{T}=\mathbf{m} \times \mathbf{B},
\end{aligned}
$$

where $\nabla$ is the gradient operator. The magnetic dipole moment can be calculated multiplying the magnetization of the material, $\mathbf{M}$, and the volume of the permanent magnet, $v$.

$$
\mathbf{m}=\mathbf{M} v .
$$

Assuming that magnetic field variations in the workspace are small enough, the magnetic force acting on the permanent magnet can be neglected. Therefore, the actuation is primarily dependent on the magnetic torque. 


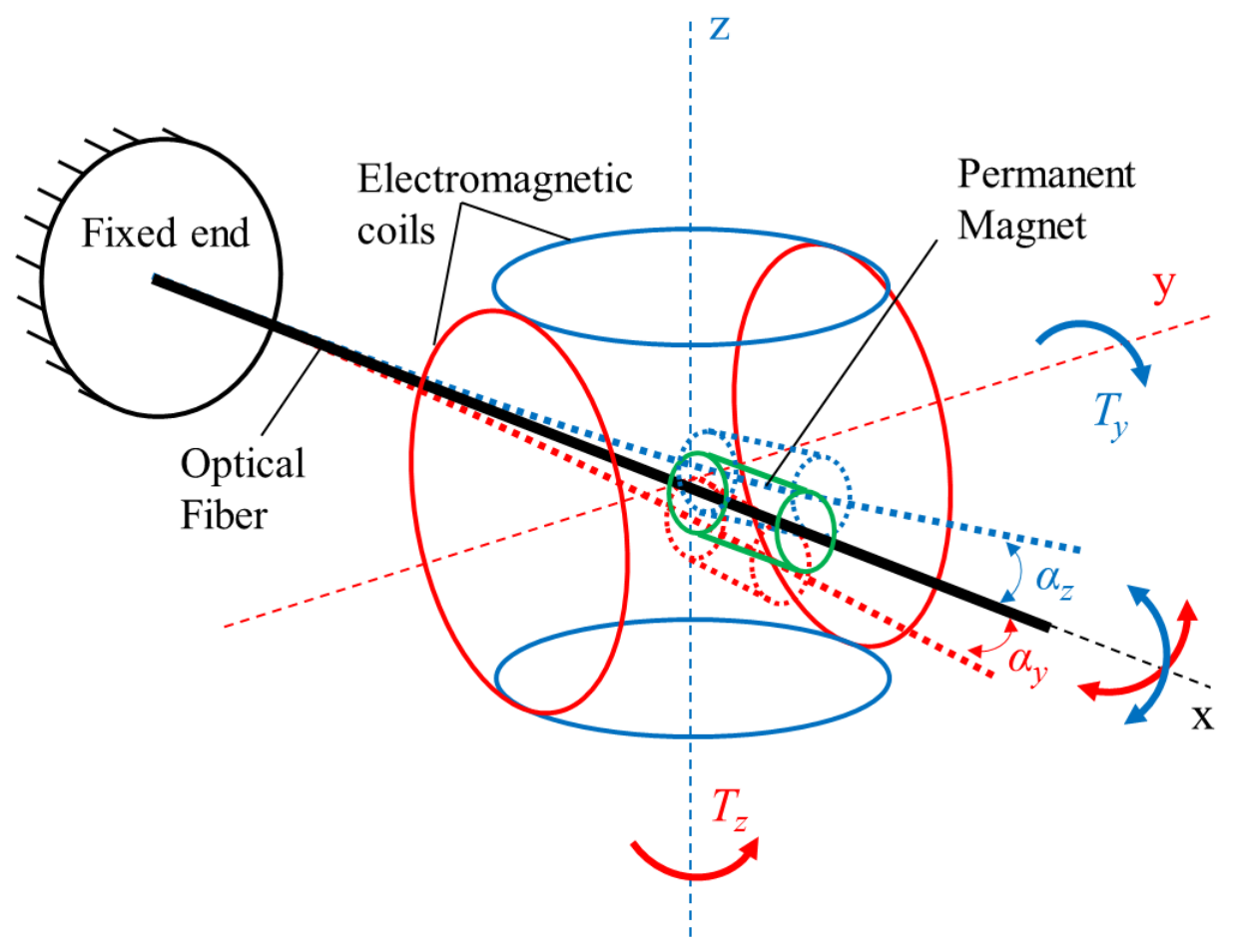

Figure 4-2: Schematically illustration of the magnetic actuation with four electromagnetic coils and permanent magnet. Electromagnetic coil pairs bend optical the fiber in $\mathrm{y}$ - and z-axes with the effect of the magnetic torques $T_{y}$ and $T_{z}$. Corresponding beam bending angles are $\alpha_{z}$ and $\alpha_{y}$, respectively.

Magnetic torque can be calculated with (4.5b) on a magnetic dipole, $\mathbf{m}=\left[m_{x}, m_{y}, m_{z}\right]$

$$
\left[\begin{array}{c}
T_{x} \\
T_{y} \\
T_{z}
\end{array}\right]=\left[\begin{array}{l}
m_{y} B_{z}-m_{z} B_{y} \\
m_{z} B_{x}-m_{x} B_{z} \\
m_{x} B_{y}-m_{y} B_{x}
\end{array}\right] .
$$

$B_{y}$ is induced by the coil pair placed in y-axis and $B_{z}$ is induced by the coil pair placed in z-axis. $B_{x}$ can be neglected as compared to the $B_{y}$ and $B_{z}$. Thus, (4.7) becomes

$$
\left[\begin{array}{c}
T_{x} \\
T_{y} \\
T_{z}
\end{array}\right]=\left[\begin{array}{c}
m_{y} B_{z}-m_{z} B_{y} \\
-m_{x} B_{z} \\
m_{x} B_{y}
\end{array}\right] .
$$




\subsubsection{Bending of a cantilevered optical fiber}

In the system design, an optical fiber is adapted to be a mechanical cantilever: the fiber is fixed at the center of the tool wall as seen in Figs. 2-1 and 4-2. A ring permanent magnet is inserted over optical fiber. Thus, optical fiber is bent with the effects of the $T_{z}$ and $T_{y}$ in $\mathrm{y}$ - and z-axes with bending angles $\alpha_{y}$ and $\alpha_{z}$, respectively.

$$
\alpha_{y, z}=\frac{T_{z, y} l}{E I_{m}}
$$

where $E$ is elasticity modulus, $I_{m}$ is moment of inertia, and $l$ is the distance between fixed end of cantilever beam and torque point.

Since the optical fiber is fixed on the internal wall of the tool (see Fig. 4-2), $T_{x}$ does not cause bending of optical fiber about x-axes.

The laser light diverges when it goes out of an optical fiber. However, focusing the laser beam in a small spot is crucial for efficient ablations. Thus, two plano-convex lenses are used in order to focus the laser beam on a target: the first lens collimates the laser light, and then the second lens focuses on the target surface (see Fig. 2-3b).

With two current inputs, $I_{y}$ and $I_{z}$, a magnetic field is created in the workspace. This creates magnetic torque on the permanent magnet attached to an optical fiber. Optical fiber is bent with the effect of the magnetic torques $T_{y}$ and $T_{z}$. Depending on the bending angle of the fiber ( $\alpha_{y}$ and $\alpha_{z}$ in Fig. 4-2), the laser spot changes its position, $d_{y}$ and $d_{z}$, on the target surface. Thus, the laser spot deflections, $d_{y}$ and $d_{z}$, on a target surface can be achieved with two current inputs $I_{y}$ and $I_{z}$, respectively.

Considering (4.3), (4.8), and (4.9), it can be seen that bending angles $\alpha_{y}$ and $\alpha_{z}$ are linearly dependent on currents of the electromagnetic coils $I_{y}$ and $I_{z}$. Both Figs. 3-5 and 3-6 presented in the previous section suggest that the system can be approximated by a second order system. Thus, the governing equation for the modeling of the two 
axes dynamics is written as

$$
\ddot{\alpha}=A \dot{\alpha}+B \alpha+C u
$$

where $\boldsymbol{A}, \boldsymbol{B}$ and $\boldsymbol{C}$ are $2 \times 2$ matrices, $\mathbf{u}=\left[I_{y}, I_{z}\right]^{\top}$ represents the current inputs, $\boldsymbol{\alpha}=\left[\alpha_{y}, \alpha_{z}\right]^{\top}$ the measured bending angles, $\dot{\boldsymbol{\alpha}}=\left[\dot{\alpha}_{y}, \dot{\alpha}_{z}\right]^{\top}$ the velocities, and $\ddot{\boldsymbol{\alpha}}=$ $\left[\ddot{\alpha}_{y}, \ddot{\alpha}_{z}\right]^{\top}$ the accelerations.

For a set of data, (4.10) is written as

$$
\ddot{\boldsymbol{\alpha}}(t)=\boldsymbol{A} \dot{\boldsymbol{\alpha}}(t)+\boldsymbol{B} \boldsymbol{\alpha}(t)+\boldsymbol{C u}(t) .
$$

Here, $\boldsymbol{\alpha}$ is the laser spot position vector computed by post-processing of recorded videos. The vector $\mathbf{u}$ is the current vector coming from measurements. $\alpha$ is derived to get the $\dot{\alpha}$ and $\ddot{\alpha}$.

\subsection{Identification and Feed-Forward Control}

\subsubsection{Identification}

The model (4.11) can be written as

$$
\ddot{\alpha}=P N,
$$

where $\boldsymbol{P}=[\boldsymbol{A}, \boldsymbol{B}, \boldsymbol{C}]$ is the coefficient matrix and $\boldsymbol{N}=[\dot{\boldsymbol{\alpha}}(t) ; \boldsymbol{\alpha}(t) ; \mathbf{u}(t)]$ is the measurement matrix. Estimating the coefficients matrix is an optimization problem: the error is minimized between the estimated and the actual response:

$$
\boldsymbol{P}^{*}=\underset{P}{\operatorname{argmin}}|\ddot{\alpha}-P \boldsymbol{N}|,
$$

where $\boldsymbol{P}^{*}$ is the estimated coefficients. A solution to the optimization problem is given by

$$
P^{*}=\ddot{\alpha} N^{+},
$$




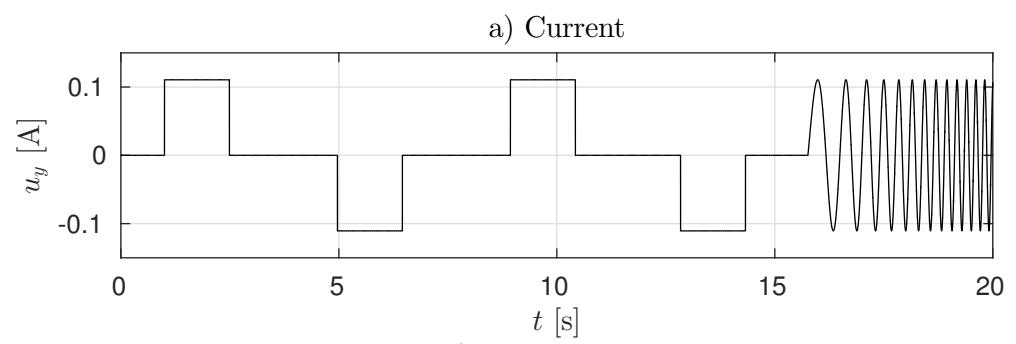

b) Bending Angle

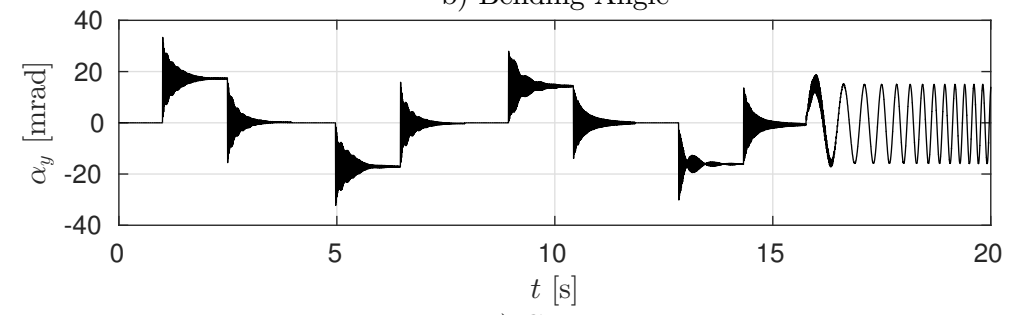

c) Current

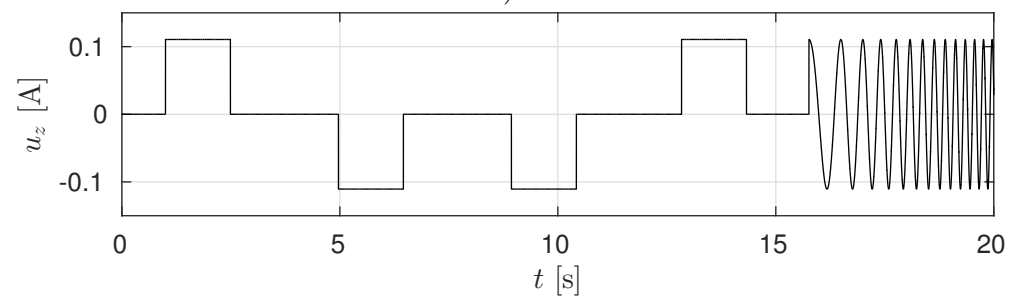

d) Bending Angle

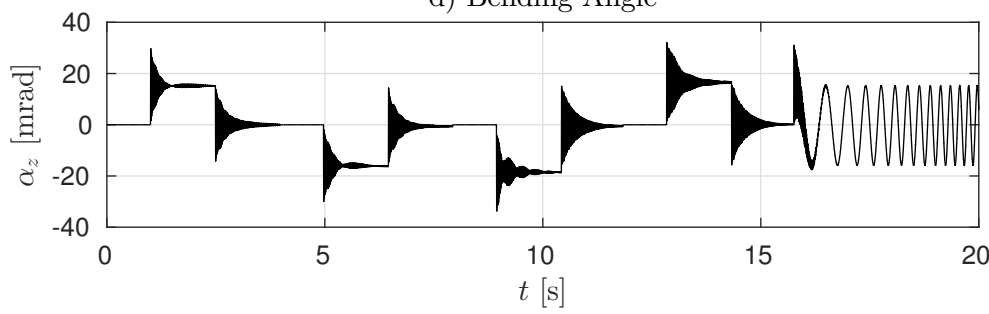

Figure 4-3: Calibration data set for the model estimation: current inputs are $u_{y}$ and $u_{z}$ in A, bending angles are $\alpha_{y}$ and $\alpha_{z}$ in mrad.

where $\boldsymbol{N}^{+}$stands for the Moore-Penrose pseudoinverse of matrix $\boldsymbol{N}$.

For the estimation of the model, four different step responses and sinusoidal current input with a varying frequency (chirp signal) were fed into the coils: these current profiles are shown in Fig. 4-3. The obtained data from experiments were used to calculate the coefficient matrix of the system with (4.14). The obtained results are

$$
\boldsymbol{P}^{*}=\left[\begin{array}{cccccc}
-193 & 9 & -1.45 E 5 & 3.28 E 3 & 2.3 E 3 & 73 \\
-4 & -192 & 4.99 E 3 & -1.45 E 5 & -184 & 2.34 E 3
\end{array}\right]
$$


Here, $\boldsymbol{P}^{*}$ presents the values of the $\boldsymbol{A}, \boldsymbol{B}$, and $\boldsymbol{C}$ matrices that are the coefficients of the velocity, position, and current input, respectively. Principally, diagonal elements of each matrix define the system behavior. Additionally, anti-diagonal elements defines the effects of the components on the opposite axis.

The estimated natural frequency can be calculated by taking into account the model of the harmonic oscillations of the second order system:

$$
\ddot{\alpha}+2 \zeta \omega_{n} \dot{\alpha}+\omega_{n}^{2} \alpha=K \cos (\omega t)
$$

where $\omega_{n}$ is the circular natural frequency, $\zeta$ is the proportional damping, and $K$ is the amplitude of the oscillations.

Natural frequency of the system can be estimated equating the coefficients of $\alpha$ in (4.10) and (4.15), $\boldsymbol{B}$ and $\omega_{n}^{2}$. $\omega_{n}$ is calculated as $380.6 \mathrm{rad} / \mathrm{s}$ and $f_{n}$ is $60.6 \mathrm{~Hz}$. This result is in agreement with the observed natural frequency of the system with a 3.8 $\%$ relative error.

\subsubsection{Feed-forward control}

A feed-forward controller for the system can be defined using the model (4.10). Given a desired trajectory, $\boldsymbol{\alpha}_{\mathbf{d}}$, the desired current input, $\mathbf{u}_{\mathbf{d}}$, can be calculated with the inverse of the model

$$
\mathbf{u}_{\mathrm{d}}=C^{-1}\left(\ddot{\alpha}_{\mathrm{d}}-A \dot{\alpha}_{\mathrm{d}}-B \alpha_{\mathrm{d}}\right)
$$

A block diagram for such feed-forward controller is presented in Fig. 4-4. After the calculation of the inverse of the model, the desired current vector is sent to the magnetic laser scanner to execute the desired trajectory.

\subsubsection{Single target point}

The aim of this experiment is to present the trajectory of laser spot given a single target point. In order to reach the target point, a sigmoid-shaped trajectory was defined between the initial position and target position of the laser spot. The defined 


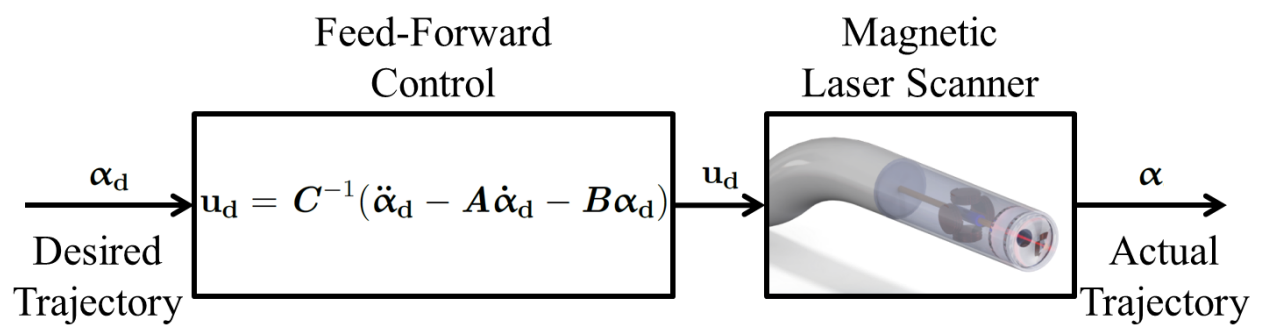

Figure 4-4: Block diagram of the feed-forward control.

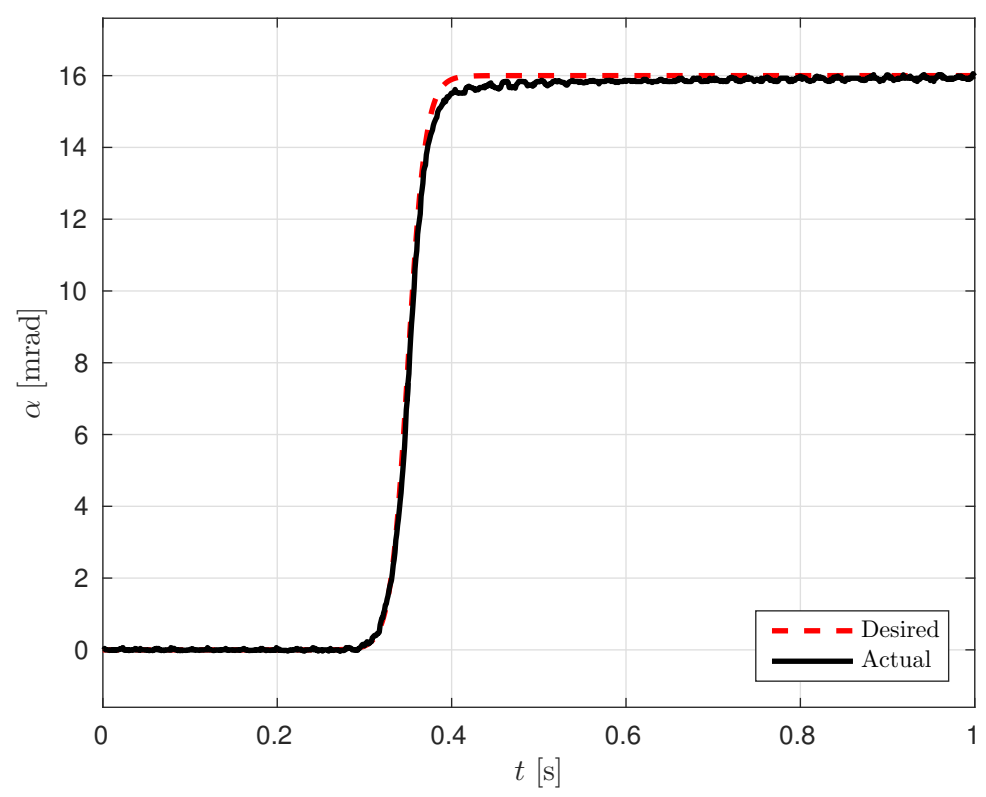

Figure 4-5: Executed actual trajectory for a sigmoid-shaped desired trajectory.

target trajectory was sent to the feed-forward controller as presented in Fig. 4-4. For the performance assessment, RMSE and maximum errors were computed.

The single set point was chosen at $1 \mathrm{~mm}(\alpha \cong 16 \mathrm{mrad})$. The reference point is initial position of the laser spot [0,0]. Fig. 4-5 presents the comparison of actual and desired trajectories. Maximum error was $0.45 \mathrm{mrad}$. The oscillations of the step response which have been presented in Fig. 3-5 are eliminated with this approach. 



\section{Chapter 5}

\section{Teleoperation User Trials}

During operations, surgeons perform tissue ablations with customized trajectories. It is essential to provide (i) accurate laser positioning and (ii) intuitive control for delicate operations. The usability and accuracy assessments of magnetic laser scanner are discussed in this chapter. Experiments with 12 subjects were performed with the setup introduced in Section 2.4. Results are presented with qualitative and quantitative assessments.

\section{$5.1 \quad$ User Trials}

The main objective of the proposed experiment is to analyse the usability and the 2D positioning accuracy of the magnetic laser scanner when controlled through a tablet device. To this end, user trials consisting of trajectory following tasks were performed. 12 subjects (11 male and 1 female, average age $27.3 \pm 3.5$ years, 10 right-handed and 2 left-handed) performed 10 trials each with 5 different target trajectories resulting in 2 repetitions for each trajectory in a randomized order. Subjects were engineering students with no prior experience in 2D laser manipulation with a tablet device. All subjects received verbal and written information describing the experiments and its goals, then provided written informed consent in accordance with recommendations from authors' institution and based on the Declaration of Helsinki.

Fig. 5-1 depicts target trajectories (s-curves, c-curves and a straight line) used in 


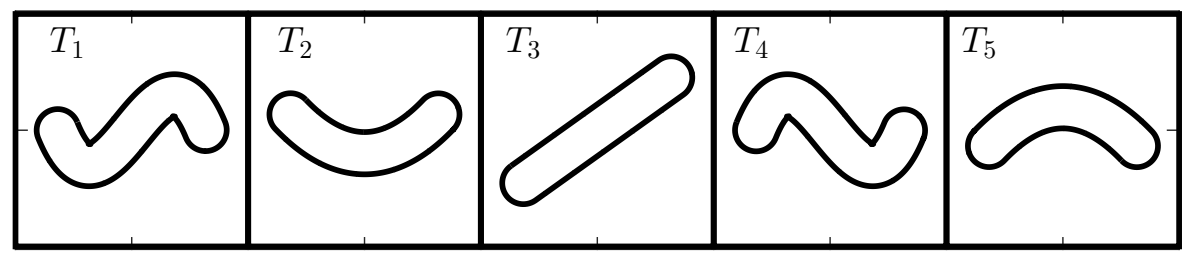

Figure 5-1: The target trajectories that are used in the experiments: $T_{1}$ and $T_{4}$ are s-curves, $T_{2}$ and $T_{5}$ are c-curves and $T_{3}$ is a straight trajectory.

the experiments. In previous studies, laser position control systems were assessed by using similar trajectories with the s-curves, c-curves and straight lines [43]. These five different trajectories increase the variety of the shapes that can be used to assess the magnetic laser scanner.

In the experimental setup, the camera observes a white paper as target plane. The target trajectories are shown on the computer screen as virtual items created by changing the pixel colors of the video frames at the target locations (Fig. 5-2). Subjects are asked to follow the target trajectories keeping the laser spot inside of the borders of the shapes. The center position of the laser spot is compared to the central line of the target trajectory for assessing the control accuracy.

\subsubsection{Subjective analysis}

The aim of this analysis is to understand if the magnetic laser scanner can be effectively used to accomplish the specific tasks. For subjective analysis of the teleoperation user trials, a dedicated questionnaire was prepared to assess the controllability and the response of the magnetic laser scanner. The questionnaire, based on the System Usability Scale (SUS) [77], focuses on the ease-of-use of the system, the fatigue induced by the control, the mental work-load, the accuracy and precision of the magnetic laser scanner, and the intuitiveness of the control. Table 5.1 presents the questionnaire items. In the questionnaire, some items have the same assessment objective, however they are expressed in a different way allowing to assess the consistency of the answers. At the end of the experimental session, subjects were presented 

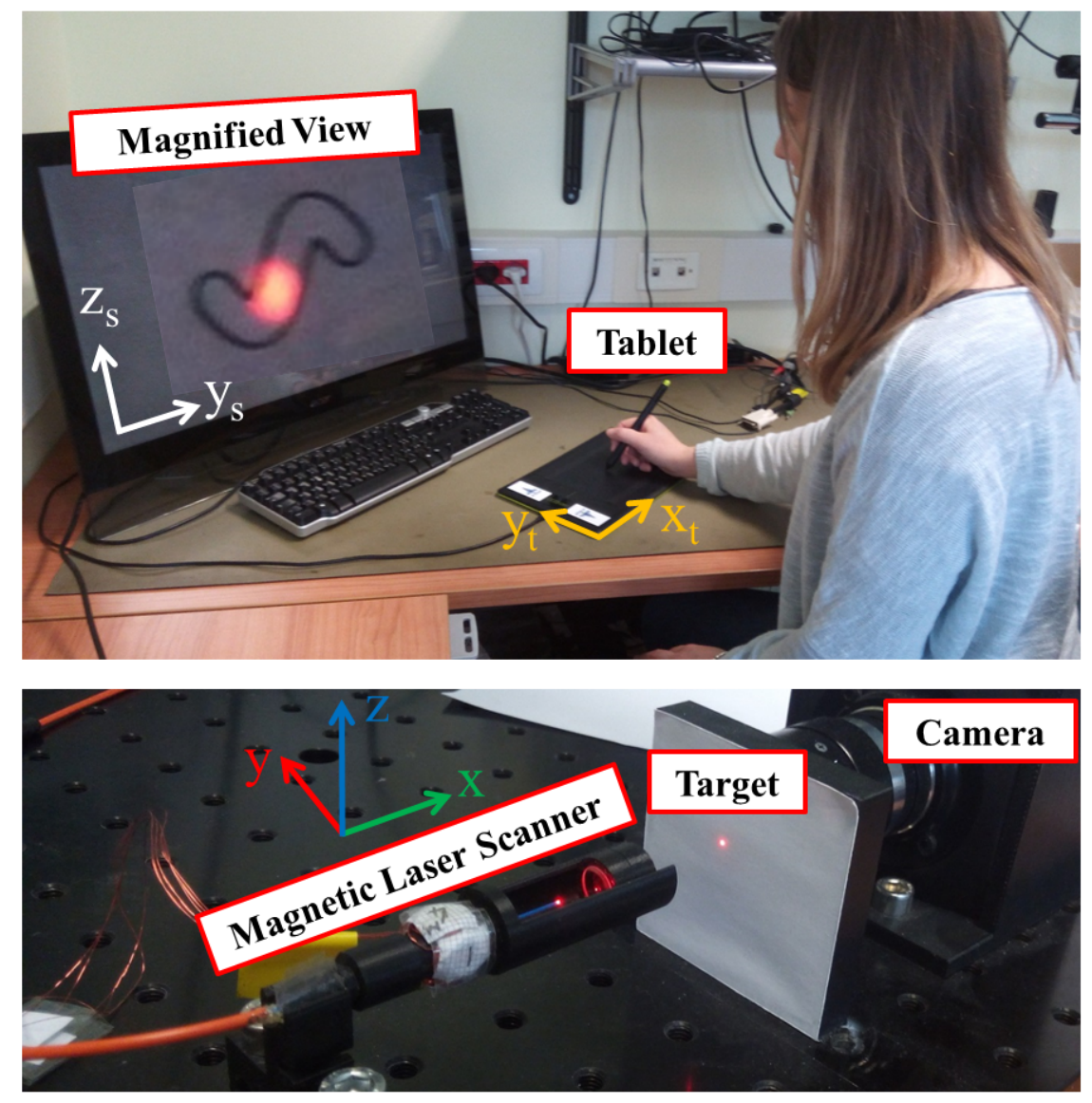

Figure 5-2: A scene from user trials with the tablet control of magnetic laser scanner. 
Table 5.1: Questionnaire Items

\begin{tabular}{cl}
\hline \hline 1 & The control of the device (laser motion) was precise. \\
2 & The control of the device (laser motion) induced fatigue in my hand. \\
3 & The control/use of the device was easy. \\
4 & I was stressed, irritated or annoyed using this device. \\
5 & I found the device was easy to learn, so I could start using it quickly. \\
6 & The laser motion was not responsive enough to accomplish the task. \\
7 & The characteristics of the device (motion, speed, control) \\
& were sufficient to accomplish the task. \\
8 & My performance (accuracy) in this task with this device was satisfying. \\
9 & It is easy to make errors with this device. \\
10 & I would like to use this device again for this kind of task.
\end{tabular}

this questionnaire on the computer screen and asked to fill out based on their experience with the device. Subjects chose their scores with a slide bar according to their agreement level with the questions (Disagree (1) - Agree (101)).

Items 2, 4, 6, and 9 are expressed with a negative statement: disagreement is expected for these questions, whereas agreement is expected for the items 1, 3, 5, 7, 8, and 10. In addition to these items, an additional question was asked to the subjects: the perceived average execution time in seconds for a single trajectory. This allowed the assessment of the perceived mental effort.

\subsection{Results}

Fig. 5-3a shows a comparison of the executed and target trajectories with the error bars. Fig. 5-3b presents the errors for this trajectory and calculated RMSE.

Fig. 5-4 depicts the RMSE for each subject over 10 trials. The average RMSE over the 12 subjects is $39 \pm 8 \mu \mathrm{m}$. These values indicate that the magnetic laser scanner also provides good accuracy when compared to the traditional systems with the manual micromanipulator considering the previously reported RMSE values (211-650 $\mu \mathrm{m})$ in the literature $[71,33,46]$.

Fig. 5-5 depicts the time required to follow the target trajectory for each subject. The average time for all subjects is 8.78 seconds. Taking into account the RMSE values (Fig. 5-4) and trajectory execution times (Fig. 5-5), it can be observed that 


\section{a) Comparison}

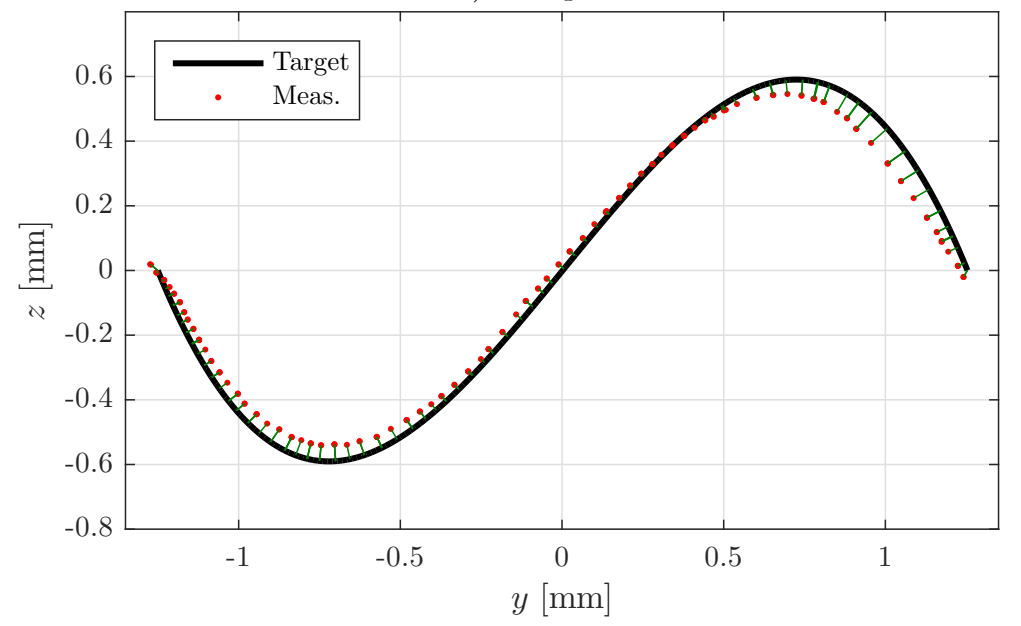

b) $\mathrm{RMSE}=35 \mu \mathrm{m}, \max (e r r)=69 \mu \mathrm{m}$

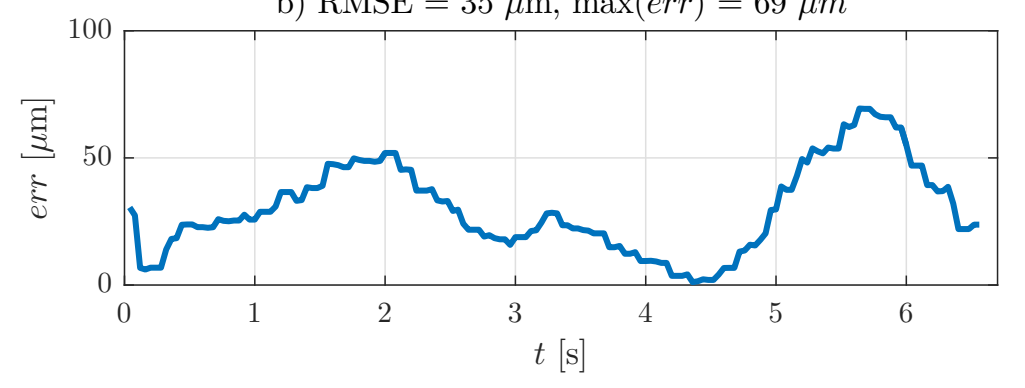

Figure 5-3: a) Comparison of a sample s-curve trajectory (red dots) against a target trajectory (black line). Green lines show the error bars for each measurement point. b) Calculated errors for all measurement points for the sample trajectory. 


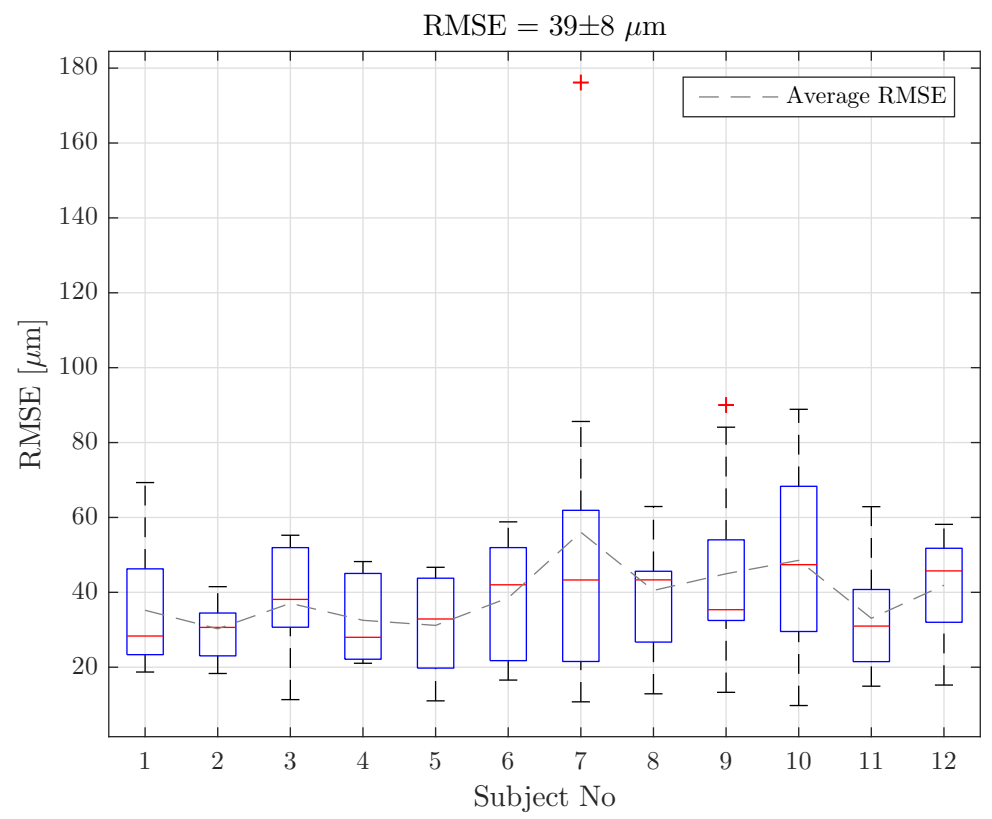

Figure 5-4: The boxplots of the RMSE for each subject over 10 trials. The outliers are plotted individually with the red '+' symbol.

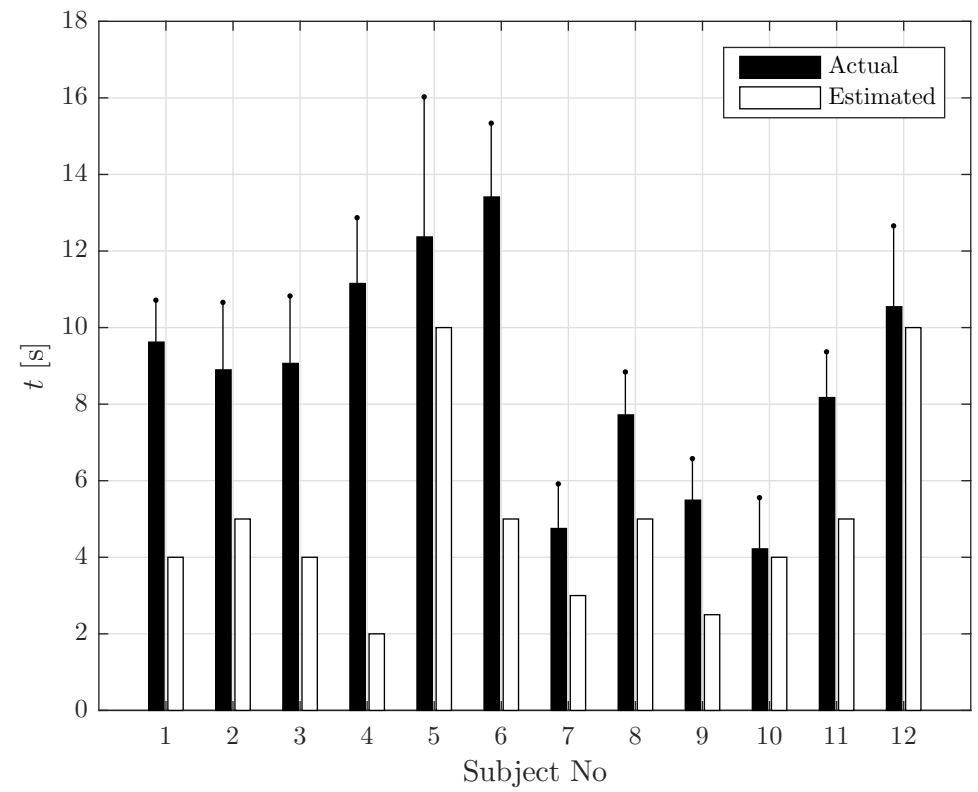

Figure 5-5: Comparison of the actual and estimated average execution times. 
slower trajectory executions lead to lower errors. For instance, subjects 2 and 4 executed the trajectories with average times of 8.9 and 11.2 seconds, respectively. The corresponding RMSE values are 30 and $33 \mu \mathrm{m}$. This shows that the system allows precise control of the laser spot. Thus, surgeons can carefully execute trajectories precisely on the desired locations. Moreover, fast trajectory executions tended to lead to larger errors as compared to the slow ones. The largest errors were observed for subjects 7, 9 and 10, who presented also the lower average trajectory execution times (RMSE values equal to 56, 45 and $48 \mu \mathrm{m}$ and times equal to 4.8, 5.5 and 4.2 seconds, respectively).

In order to assess the learning during the experiments, Figs. 5-6 and 5-7 are presented for error and time evaluation for each trial. The average values of the RMSE and maximum errors are calculated over 12 subjects for each trial and results are presented in Fig. 5-6. RMSE and maximum errors have similar behavior and both decrease with the trial number. The maximum error for the first trial is $108 \mu \mathrm{m}$ and for the last trial, $71 \mu \mathrm{m}$. For the average RMSE, first and last values are 49 and 33 $\mu \mathrm{m}$, respectively. The general behavior of the error shows that subjects are improving their precision during the experiments.

Fig. 5-7 depicts the average time evaluation over 10 trials. The results show that the average time decreases by 1.1 seconds. The system allows increased ease-of-use even with a little training.

Subjective Analysis: Fig. 5-8 depicts the questionnaire results for the items presented in Table 5.1. It is observed that the overall assessment is very positive for the device. Specifically, the following points are noted:

- The ease-of-use: High scores on items 3, 5, and 10 indicate that the system is easy to use and can be learned easily.

- The fatigue: Low scores on items 2 and 4 shows that the control method does not induce fatigue in the hand.

- The mental work load: The scores of the items 3, 4, and 5 indicate that the control of the system does not require high mental effort. The required cognitive 


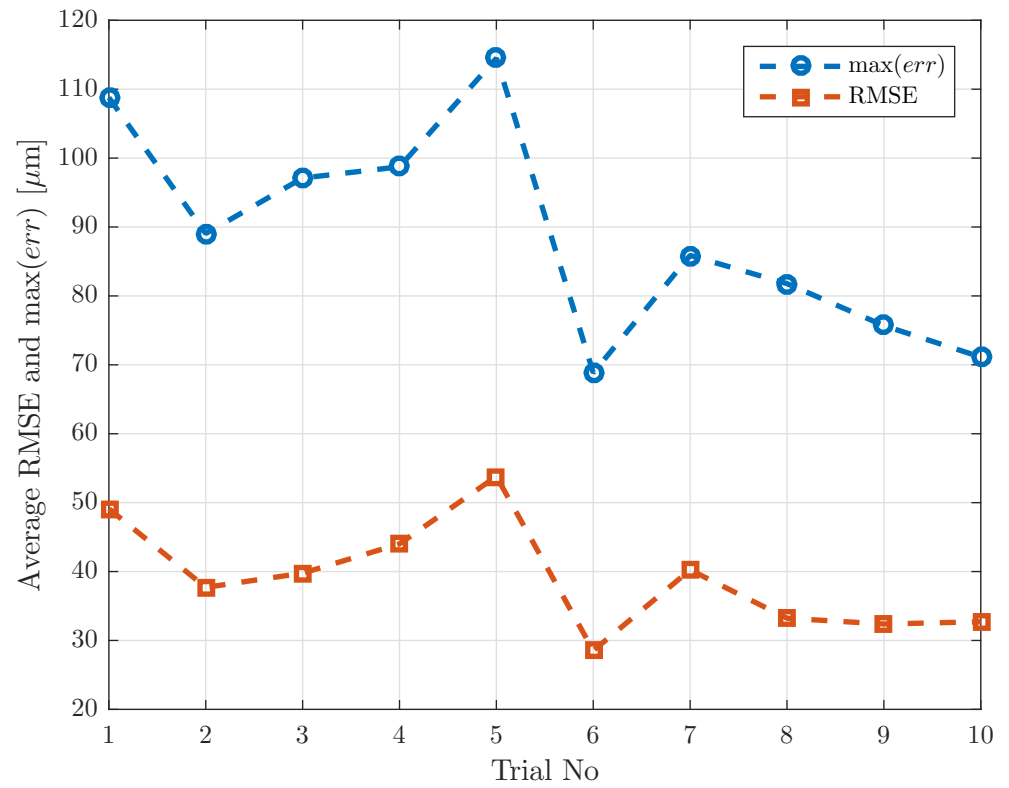

Figure 5-6: Overall average RMSE and maximum errors for each trial considering the data from all experimental subjects.

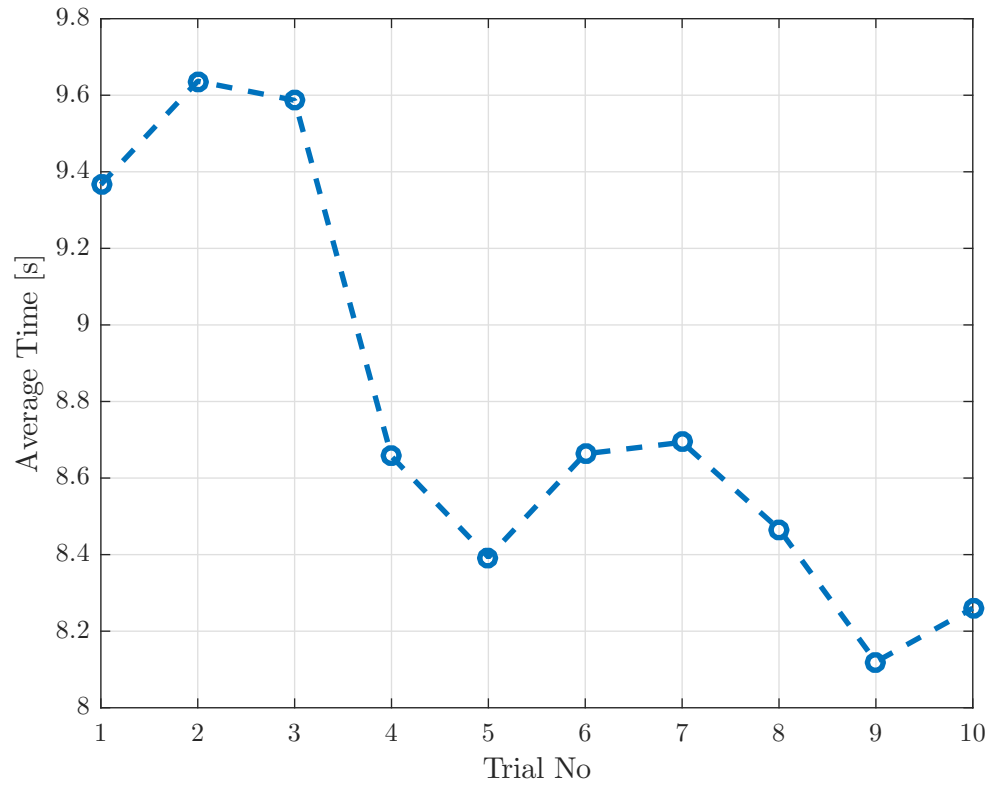

Figure 5-7: Average time for each trial over 12 subjects. 
work load is not high.

- The accuracy and precision: The items 1 and 7 have high scores and the item 6 and 9 low scores: indicating that users assess the magnetic actuation as accurate and precise for the execution of laser positioning tasks.

- Intuitiveness: The scores of the items 3, 4, and 10 indicate that the control of the magnetic laser scanner is intuitive.

- Self-assessment: The high score of the item 8 shows that subjects felt confident in using the system.

The estimation of elapsed time to complete a specific task can provide an indication of the perceived mental workload for that task [78]. For self-assessment of this perceived mental workload, subjects were asked to estimate an average execution time for their performances after 10 trials. In Fig. 5-5, actual execution times are compared with the estimated execution times. As can be seen, the estimated execution times are lower than the actual execution times. This indicates that, even though the actual effort may be high, the perceived mental effort to use the system is low. This is a positive assessment for the system.

\subsection{Discussion}

The results presented above show that the new concept of teleoperated endoscopic laser manipulation improves the accuracy of the laser control compared to the stateof-the-art laser manipulation technique based on manual micromanipulators. Accuracy assessment of operations with the magnetic laser scanner shows that average RMSE on trajectory tracing tasks are less than $40 \mu \mathrm{m}$. This value compares positively with respect to RMSE values in the range 211-650 $\mu \mathrm{m}$ reported for the manual micromanipulator with $400 \mathrm{~mm}$ operating distance [71, 33, 46]. Thus, endoscopic laser control provides at least five times better accuracy than traditional laser manipulation techniques due to: 1) shorter operating distance; 2) high-resolution laser 


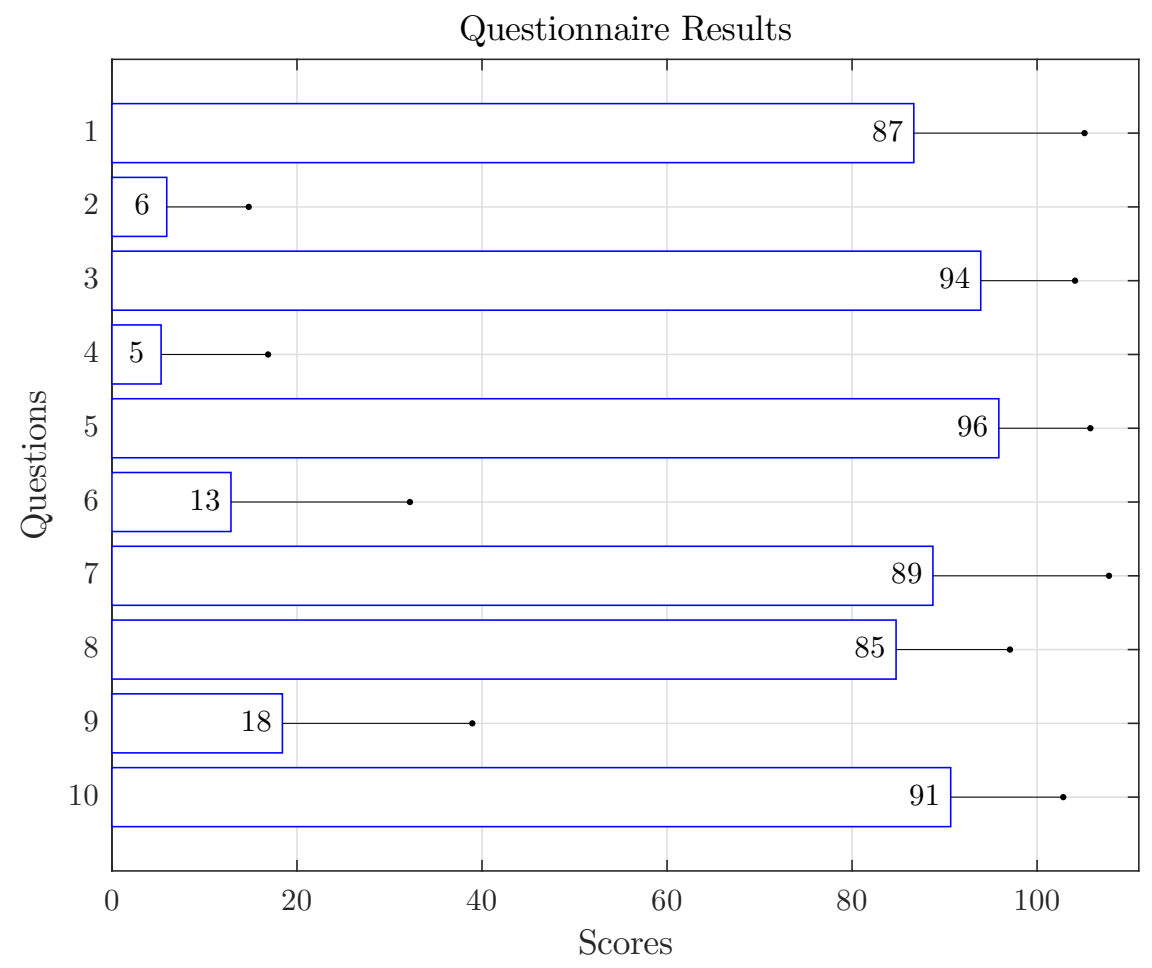

Figure 5-8: Questionnaire results

micromanipulation capabilities; and 3) the use of teleoperation techniques, including motion scaling and better user interface.

\subsection{Conclusion}

Performance of the proof-of-concept device has been assessed with characterization experiments and teleoperation user trials. The magnetic laser scanner demonstrated to be able to perform stable scanning motions up to $40 \mathrm{~Hz}$, and to provide high repeatability with an error around $20 \mu \mathrm{m}$. In addition, the prototype has been integrated with a tablet device for teleoperation. The accuracy of such integrated system has been assessed through user trials involving 12 subjects. Results showed that target trajectories can be executed with $39 \pm 8 \mu \mathrm{m}$ accuracy, which is at least five times better than reported values regarding current state-of-the-art laser systems. Furthermore, subjective assessments indicated that the proposed system is intuitive and easy to control, presenting good response to tablet commands. 


\section{Chapter 6}

\section{Automated Trajectory Executions}

In surgical operations, deep laser incisions are performed on customized trajectories with repetitive passes. After defining desired trajectories with a tablet device, the laser manipulation system must perform the desired trajectory accurately. In this chapter, accuracy assessment of the magnetic laser scanner is performed considering predefined trajectories with different sizes and speeds. Validation experiments are performed with the feed-forward controller derived in Chapter 4. Results are discussed based on the achieved laser position accuracy and bending angles of the cantilever beam.

\subsection{D Trajectory Executions}

In order to validate the designed feed-forward controller, 2D trajectories were defined considering different trajectory types, scanning speeds and amplitudes. Desired trajectories were used to calculate the current values required to achieve them using the inverse of the estimated model (4.16). Calculated current values were sent to the magnetic laser scanner to execute the desired trajectories. The actual trajectories executed by the system were recorded and compared with the desired ones. The memory of the high-speed camera that is used in the experiments allows recording the frames for 2 seconds at high resolution. 

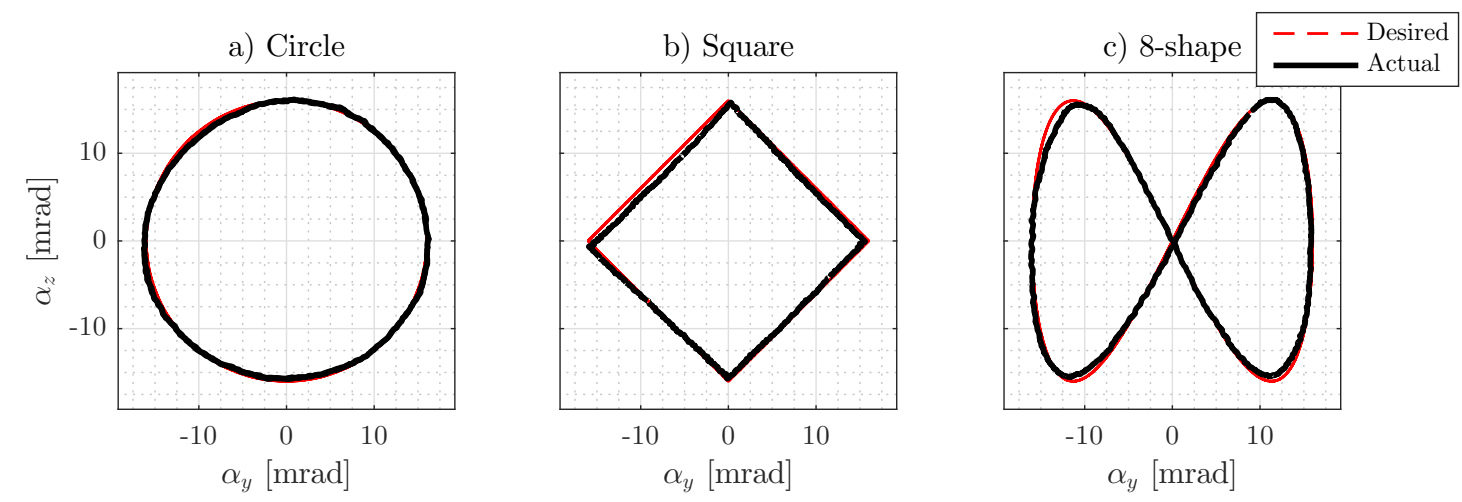

Figure 6-1: Validation experiments for different trajectory types a) Circle, b) Square, and c) 8-shape. Error values for validation experiments are presented in Table 6.1.

\subsubsection{Trajectory tracing}

The aim of the experiment is to demonstrate that the system can perform any $2 \mathrm{D}$ trajectory on the target plane. In this experiment, a circle, a square, and a 8-shaped desired trajectories are automatically performed by the magnetic laser scanner. Each experiment was performed for 1 second at $1 \mathrm{~Hz}$. For comparison of the actual and desired trajectories, RMSE and maximum errors were calculated.

Fig. 6-1 presents a comparison of the desired and actual trajectories. Table 6.1 presents RMSE values and maximum errors for the experiments. For circular trajectory, RMSE value is $0.2 \pm 0.1 \mathrm{mrad}$ and maximum error is $0.6 \mathrm{mrad}$. For square trajectory, RMSE value is $0.4 \pm 0.2 \mathrm{mrad}$ and maximum error is $0.9 \mathrm{mrad}$. For 8shaped trajectory, RMSE value is $0.4 \pm 0.2 \mathrm{mrad}$ and maximum error is $1.1 \mathrm{mrad}$. Error values for the laser spot deflections on the target plane for $30 \mathrm{~mm}$ focal length are: for circular trajectory, RMSE value is $15 \pm 8 \mu \mathrm{m}$ and maximum error is $35 \mu \mathrm{m}$; for square trajectory, RMSE value is $26 \pm 14 \mu \mathrm{m}$ and maximum error is $57 \mu \mathrm{m}$; and for 8 shaped trajectory, RMSE value is $22 \pm 13 \mu \mathrm{m}$ and maximum error is $69 \mu \mathrm{m}$.

\subsubsection{Scanning frequency}

This experiment aims to characterize maximum speed that the magnetic laser scanner can execute $2 \mathrm{D}$ trajectories within $\pm 1 \mathrm{mrad}$ error range (5-6\% of trajectory size). 
In this experiment, $2 \mathrm{~mm}$ diameter circular trajectories were performed for 2 seconds with scanning speeds 1,5 , and $15 \mathrm{~Hz}$. The corresponding repetition times are 2, 10, and 30 passes for 1, 5 and $15 \mathrm{~Hz}$, respectively. Comparison of this experiments is based on a perfect circular trajectory with $2 \mathrm{~mm}$ diameter.

Fig. 6-2 depicts the results of the scanning speed experiments. Table 6.1 presents RMSE values and maximum errors for the experiments. For $1 \mathrm{~Hz}, \mathrm{RMSE}$ value is $0.4 \pm 0.2 \mathrm{mrad}$ and maximum error is $1.0 \mathrm{mrad}$. For $5 \mathrm{~Hz}$, RMSE value is $0.4 \pm 0.2$ mrad and maximum error is $0.9 \mathrm{mrad}$. For $15 \mathrm{~Hz}, \mathrm{RMSE}$ value is $0.5 \pm 0.2 \mathrm{mrad}$ and maximum error is $1.1 \mathrm{mrad}$. Error values for the laser spot deflections on the target plane for $30 \mathrm{~mm}$ focal length are: for $1 \mathrm{~Hz}$, RMSE value is $27 \pm 15 \mu \mathrm{m}$ and maximum error is $59 \mu \mathrm{m}$; for $5 \mathrm{~Hz}$, RMSE value is $26 \pm 12 \mu \mathrm{m}$ and maximum error is $53 \mu \mathrm{m}$; and for $15 \mathrm{~Hz}$, RMSE value is $30 \pm 14 \mu \mathrm{m}$ and maximum error is $69 \mu \mathrm{m}$.

\subsubsection{Amplitude}

In this experiment, circular trajectories with different amplitudes are executed with the proposed feed-forward controller. Desired trajectories have 1, 2, and $3 \mathrm{~mm}$ diameter. All desired trajectories were executed with $2 \mathrm{~Hz}$ for 2 seconds: corresponding repetition is 4 times.

Fig. 6-3 shows the results of the experiments and Table 6.1 presents corresponding
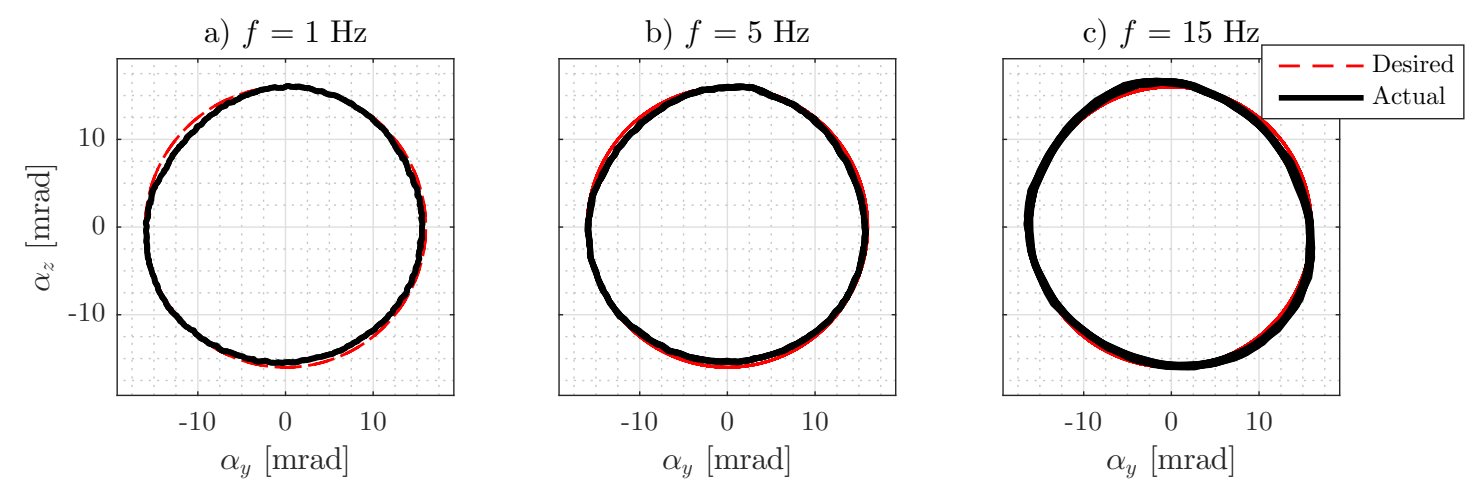

Figure 6-2: Validation experiments for different speeds with a) $1 \mathrm{~Hz}-2$ passes, b) 5 $\mathrm{Hz}-10$ passes, and c) $15 \mathrm{~Hz}-30$ passes. 
Table 6.1: RMSE values and maximum errors for validation experiments

\begin{tabular}{|l|c|c|}
\hline \multicolumn{3}{|c|}{ Fig. 6-1 } \\
\hline \multicolumn{3}{|c|}{ Trajectory Tracing } \\
\hline & RMSE & $\begin{array}{c}\max \\
(\mathrm{err})\end{array}$ \\
\hline Circle & $0.2 \pm 0.1$ & 0.6 \\
\hline Square & $0.4 \pm 0.2$ & 0.9 \\
\hline 8-shaped & $0.4 \pm 0.2$ & 1.1 \\
\hline
\end{tabular}

\begin{tabular}{|l|c|c|}
\hline \multicolumn{3}{|c|}{ Fig. 6-2 } \\
\hline \multicolumn{2}{|c|}{ Scanning Frequency } \\
\hline & RMSE & $\begin{array}{c}\max \\
(\mathrm{err})\end{array}$ \\
\hline $1 \mathrm{~Hz}$ & $0.4 \pm 0.2$ & 1.0 \\
\hline $5 \mathrm{~Hz}$ & $0.4 \pm 0.2$ & 0.9 \\
\hline $15 \mathrm{~Hz}$ & $0.5 \pm 0.2$ & 1.1 \\
\hline
\end{tabular}

\begin{tabular}{|c|c|c|}
\hline \multicolumn{3}{|c|}{ Fig. 6-3 } \\
\hline & RMSE & $\begin{array}{c}\max \\
(\text { err })\end{array}$ \\
\hline $1 \mathrm{~mm}$ & $0.3 \pm 0.1$ & 0.6 \\
\hline $2 \mathrm{~mm}$ & $0.4 \pm 0.2$ & 0.9 \\
\hline $3 \mathrm{~mm}$ & $0.7 \pm 0.3$ & 1.4 \\
\hline
\end{tabular}

All values are in mrad.

errors. For $1 \mathrm{~mm}, \mathrm{RMSE}$ value is $0.3 \pm 0.1 \mathrm{mrad}$ and maximum error is $0.6 \mathrm{mrad}$. For $2 \mathrm{~mm}$, RMSE value is $0.4 \pm 0.2 \mathrm{mrad}$ and maximum error is $0.9 \mathrm{mrad}$. For $3 \mathrm{~mm}$, RMSE value is $0.7 \pm 0.3 \mathrm{mrad}$ and maximum error is $1.4 \mathrm{mrad}$. Error values for the laser spot deflections on the target plane for $30 \mathrm{~mm}$ focal length are: for $1 \mathrm{~mm}$, RMSE value is $20 \pm 7 \mu \mathrm{m}$ and maximum error is $36 \mu \mathrm{m}$; for $2 \mathrm{~mm}$, RMSE value is $23 \pm 13 \mu \mathrm{m}$ and maximum error is $58 \mu \mathrm{m}$; and for $3 \mathrm{~mm}$, RMSE value is $46 \pm 22 \mu \mathrm{m}$ and maximum error is $86 \mu \mathrm{m}$.
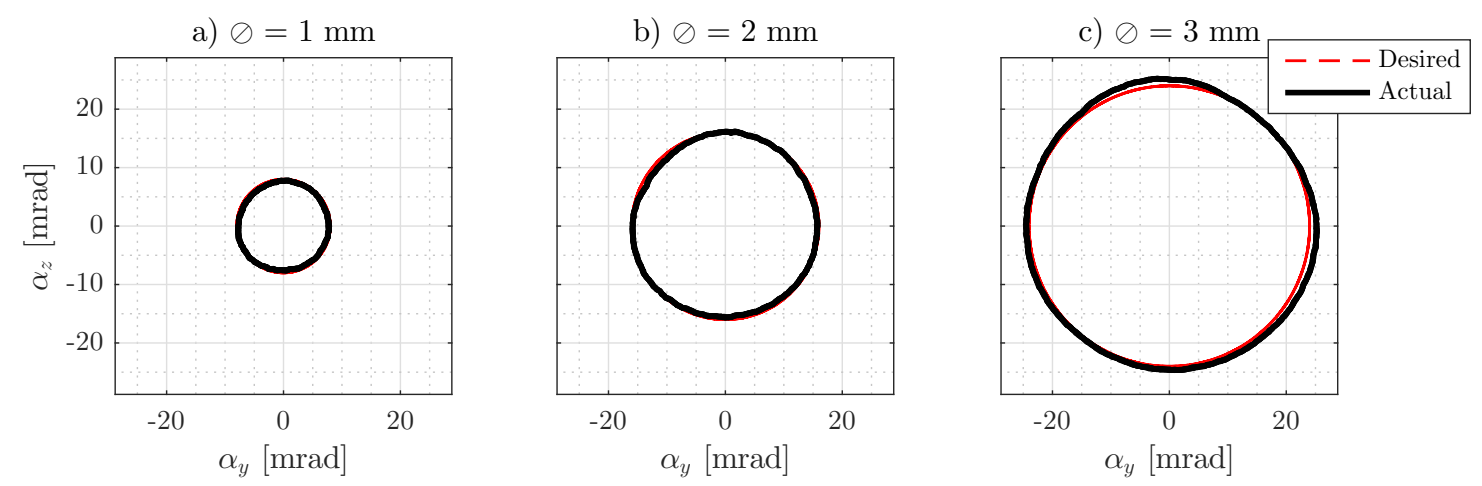

Figure 6-3: Validation experiments with different amplitudes a) $\varnothing=1 \mathrm{~mm}, \mathrm{~b}) \varnothing=$ $2 \mathrm{~mm}, \mathrm{c}) \varnothing=3 \mathrm{~mm}$. 


\subsubsection{Hysteresis}

Generally, magnetically actuated systems suffer from hysteresis which is the position discrepancy for loading and unloading conditions. In order to investigate the hysteresis of the magnetic laser scanner, the system has been actuated with the sinusoidal inputs with 8 repetitions in order to perform a straight line.

In order to show the system's position response for loading and unloading conditions, a straight line was set as a desired trajectory and repeated 8 times. Fig. 6-4 depicts the results of this experiment. Position response of magnetic laser scanner is indistinguishable for repetitive passes and loading and unloading the system with same input values. For this experiment, RMSE value based on perfect straight line is $0.2 \pm 0.1 \mathrm{mrad}$ and maximum error is $0.5 \mathrm{mrad}$. Error values for the laser spot deflections on the target plane for $30 \mathrm{~mm}$ focal length are that RMSE is $11 \pm 6 \mu \mathrm{m}$ and maximum error is $29 \mu \mathrm{m}$.

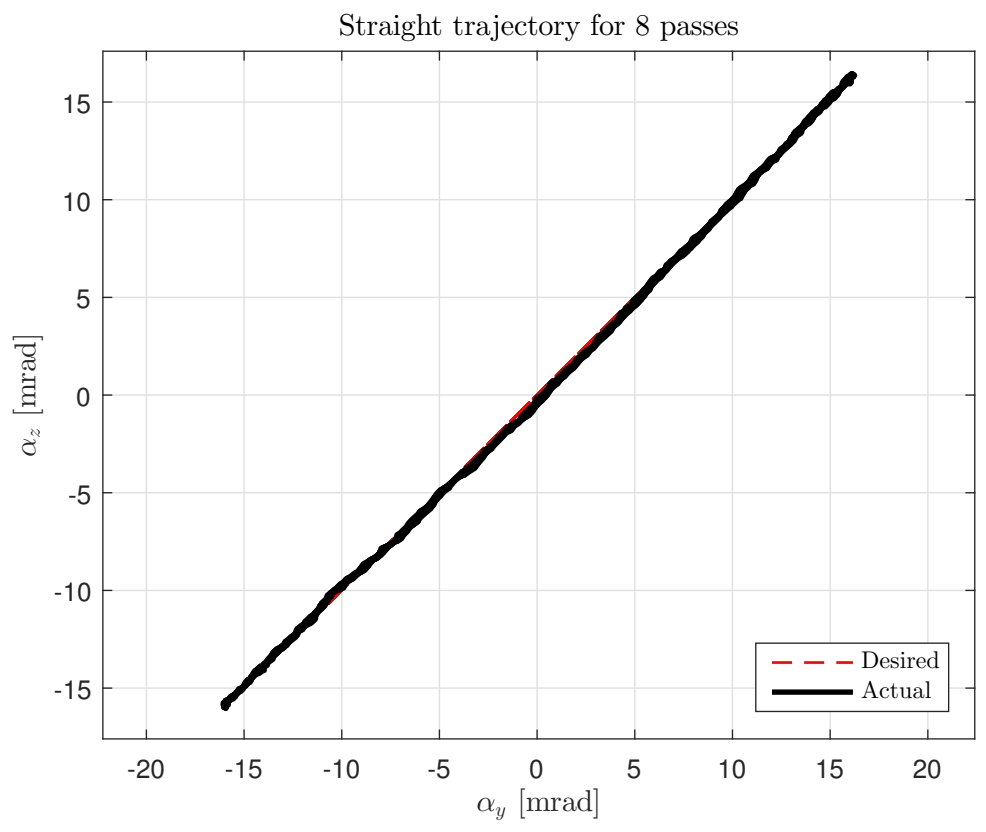

Figure 6-4: 2D straight trajectory in yz-plane for 8 passes in order to present system position response for loading and unloading conditions. 


\subsection{Discussion}

The experiments presented here demonstrated the magnetic laser scanner is able to perform precise and repeatable incisions. These two metrics are important for minimally invasive surgery. Precision is important for the safety of the operations. In order to avoid damage to the surrounding healthy tissue, laser spot should travel exactly on the desired path for repetitive ablations. Here, reported RMSE values and maximum errors were less than 0.5 and $1.4 \mathrm{mrad}$, respectively (Table 6.1). For a $30 \mathrm{~mm}$ focusing distance, corresponding RMSE values and maximum errors were measured as 32 and $90 \mu \mathrm{m}$. For $15 \mathrm{~Hz}$ in the fast scanning mode, the maximum absolute error values are less than $1.1 \mathrm{mrad}$ (about $70 \mu \mathrm{m}$ ).

Presented error values can be compared to typical laser spot diameter and resection margin of malignant tissue. For delicate microsurgery, laser spot diameter is generally in the range of 200-250 $\mu \mathrm{m}$. Errors caused by the actuation system should be much smaller than the laser spot diameter. For the proposed system, $90 \mu \mathrm{m}(1.4$ mrad) maximum error is less than $50 \%$ of typical laser diameter. Additionally, resected malignant tissue must have $1-2 \mathrm{~mm}$ resection margin to guarantee that the edges of the removed tissue are clear of cancer cells [79]. Positive margins (existence of malignant tissue on the edge) normally requires further operation for the complete removal of the cancer cells from body [80]. Considering such resection margins, the maximum errors that magnetic laser causes are less than $10 \%$ of them. However, it must be noted that reported error values in $\mu \mathrm{m}$ units are for $30 \mathrm{~mm}$ distance from tissue (Focal length of the focusing lens $=30 \mathrm{~mm}$ ). Errors linearly increase/decrease for longer/shorter focal lengths.

Maximum scanning frequency is $15 \mathrm{~Hz}$ when the errors are restricted to $\sim 1 \mathrm{mrad}$ (Fig. 6-2). Corresponding linear speed is $94 \mathrm{~mm} / \mathrm{s}$ for $15 \mathrm{~Hz}$ experiment. Scanning frequencies depend on the mechanical properties of the optical fiber cantilever beam and can be easily tuned for specific applications changing the elasticity (fiber type), or the length of the cantilever. Stiffer cantilever beams lead higher natural frequencies. Thus, stable working range for scanning speed can be increased. However, maximum 
laser spot deflections decrease due to the smaller bending angle with stiffer cantilever beams if the same actuation current values are fed to the electromagnetic coils. In the proposed design, magnetic laser scanner provides $4 \times 4 \mathrm{~mm}^{2}$ workspace that is a design choice considering the reported values for scanning range in the literature $[23,24]$. Another solution can be proposed is increasing the stiffness and the magnetic field strength at the same time in order to keep the same range of the workspace. In this case, overall system dimensions would likely increase since a thicker wire or a higher number of turns would be needed to increase the magnetic field strength.

\subsection{Conclusion}

In this chapter, the identified model (in Section 4.2.1) was used to perform feedforward control for accurate laser positioning. Results were presented with the errors of the laser positioning and bending angle of the cantilevered optical fiber. Results show that magnetic laser scanner executes trajectories with $90 \mu \mathrm{m}(1.4 \mathrm{mrad})$ accuracy. Maximum scanning frequency is $15 \mathrm{~Hz}$ when the errors are restricted to $\sim 1 \mathrm{mrad}$. The presented feed-forward controller can be seem as an alternative to visual-servoing algorithms. However, it can also be used to increase the robustness and speed of the visual-servoing algorithms by providing an estimate for the laser position on the target surface. 



\section{Chapter 7}

\section{Laser Incision Depth Control}

\subsection{Introduction}

Technological progress has transformed lasers in versatile tools for the treatment of diverse pathologies affecting delicate human organs. Together with other important applications such as eye surgery, transoral surgery is a major application field for lasers. Transoral laser microsurgery (TLM) is an evolution of the early techniques for laser surgery of the larynx. It now includes a suite of minimally invasive endoscopic techniques for the excision of minuscule laryngeal diseases [18]. In degenerative diseases associated with cancer, the primary objective of TLM is to ensure a complete eradication of the malignant tissue. At the same time, surgeons try to minimize the removal of healthy tissues, to preserve as much organ function as possible [81]. The execution of such accurate resections requires the use of a microscope and precise control of the laser incisions.

In today's surgical practice, laser incisions are performed manually, i.e., moving a free-beam laser by means of a joystick-like device, called laser micromanipulator [18]. The micromanipulator is coupled to the operating microscope, forming an effective surgical device - yet, it is difficult to master, especially because it breaks the hand-eye coordination of the surgeon [46]. In addition, presently available technologies for TLM do not include any support for sensing the depth of laser cuts. The quality of incisions relies entirely on the dexterity and visual perception of clinicians. Extensive training 
is required to develop an effective laser cutting technique, which includes both (i) the acquisition of basic knowledge of the physical principles behind laser ablation of tissue; and (ii) the ability to manipulate the laser parameters and its exposure time in order to provide accurate cutting $[18,81]$. Parameters traditionally used in TLM include power, energy delivery mode, pulse duration and incision scanning frequency. The resulting incision depends on the combination of these parameters plus the total time of laser exposure. However, it is not evident how to regulate these parameters in order to achieve the desired cutting level. While experienced clinicians normally have sufficient knowledge and understanding of the laser ablation processes, lack of experience represents a practical problem for many others.

The limitation in terms of laser beam and surgical tools controllability in transoral surgeries has recently stimulated new research and technological developments towards robot-assisted systems for these procedures. For example, the da Vinci robot (Intuitive Surgical Inc., USA) has been used and is currently certified for operations in the mouth and throat. Studies have shown that the system brings many advantages to the procedures, including tremor filtering and increased dexterity through its articulated instruments [82]. However, the use of this robot is limited by its large dimensions, long setup time, and high costs for the patient. Some of these limitations have been eliminated by flexible robotic systems developed specially for transoral surgeries, such as the Flex robot [83] and the RoboELF, a robotized endoscope for laryngeal surgery [84]. All of these systems can be used with fiber-coupled lasers, allowing enhanced controllability and access to laser-based surgeries. Nevertheless, the control of the laser dosimetry parameters is still performed by the surgeons without any assistance.

One important current limitation of fiber-based lasers for surgery is their poor focus control and requirement to work on contact with tissue to produce incisions. This translates into poor quality incisions with higher levels of thermal damage to surrounding normal tissue when compared to free-beam surgery. Consequently, the use of free-beam surgical lasers is still preferred for delicate operations such as vocal cord surgery, and robot-assisted systems have been created specifically for these pro- 
cedures $[46,33,71]$. Such technologies allow clinicians to control the laser position on the tissue through a teleoperated robotic device that replaces the traditional micromanipulator. Support is provided for motion scaling and tremor filtering, enabling more precise laser positioning. The control of laser aiming is further facilitated by the availability of user-defined motion patterns, which can be performed automatically by the system. A natural extension of these technologies is the automation of the laser incision process, based on high-level commands imparted by the surgeon.

Modeling laser tissue interactions for automated laser ablations in hard $[85,86,87$, $88]$ and soft $[89,90]$ tissue has been a field of interest in recent years. Controlled laser ablations on bone tissue with preplanned trajectories were performed using a robotic arm with $0.5 \mathrm{~mm}$ positioning error [85]. In another study, a simulation of the hard tissue ablation was developed to plan and predict the volume of the ablated tissue [86]. Moreover, incision depth was controlled intra-operatively using the inline coherent imaging which enables to measure current depth and terminate incision to achieve required depth [87]. Stopp et al. investigated the relation between laser energy, speed of laser motion and resulting ablation depth and compared their experimental results also to the theoretical model [88]. Bay et al. reported that real-time incision depth control on soft tissues can be achieved with the detection of shock waves collected from multiple acoustic detectors [89]. More recently, Fichera et al. presented that the depth of a laser incision on soft tissue can be controlled regulating the exposure time of the laser [90]. The approach assumes a fixed laser motion pattern, i.e., repeated laser scans along a straight trajectory, at constant scanning frequency. Its applicability to different incision patterns (e.g., curved lines) and for different values of laser speed is yet to be explored. As a first step in this direction, this work reports on a study aiming to model how the laser motion influences the resulting incision depth.

The effects of diode lasers on the width and depth of incisions on oral soft tissue have been studied and reported by Goharkhay [91]. These experiments were based on a fixed speed of motion of the laser and concluded that the produced incision depth is only a dependent paramater of the average power of the laser [91]. In another study, researchers compared the effects of a $805 \mathrm{~nm}$ diode laser and a $1064 \mathrm{~nm}$ NdYag laser 
on soft tissue measuring the depths and the thermal damage of the incisions [92]. These studies provided a detailed overview on histological examinations, but the analysis of laser-tissue interactions were limited to the establishment of the clinical applicability of the lasers being tested. The analysis of the correlation between the laser parameters and their effects on soft tissue, including the laser scanning speed, the number of passes and the energy density, is still a remaining task to investigate. Building up a model with these physical quantities is essential for the development of an effective robot-assisted system for laser microsurgery.

Here, the concept of a technology for the automation of the laser incision process during TLM is presented. The idea is to create a controller capable of commanding a robotic device to automatically perform incisions of given depth, based on inputs provided by the surgeon. The objective is to overcome the difficulties associated with the manual regulation of the laser parameters, enabling a more intuitive user control on the laser incision depth. A theoretical model is derived, whose validity is assessed through controlled laser experiments which are performed to understand the effects of the parameters on incision depth.

\subsection{Background}

The automation of the laser incision process is based on a recently developed system for laser-based microsurgery, called the " $\mu$ RALP Surgical System" [46]. As explained later in this section, the technology that is proposed constitutes an add-on component of this system. The $\mu$ RALP system (Fig. 7-1a) is inspired by the current setup of TLM, of which it represents a revised, computer-assisted version. Here, the traditional micromanipulator is replaced by a graphics tablet, which enables a more intuitive (writing-like) control of the laser position. The commands imparted by the surgeon through this device are mapped into corresponding laser beam trajectories on the surgical site. The controlled motion of the laser is provided by a microrobotic system, based on a fast steering mirror [71]. The microscope is replaced by a stereoscopic headmount display (HMD), which presents a magnified, 3D visualization of the surgical 


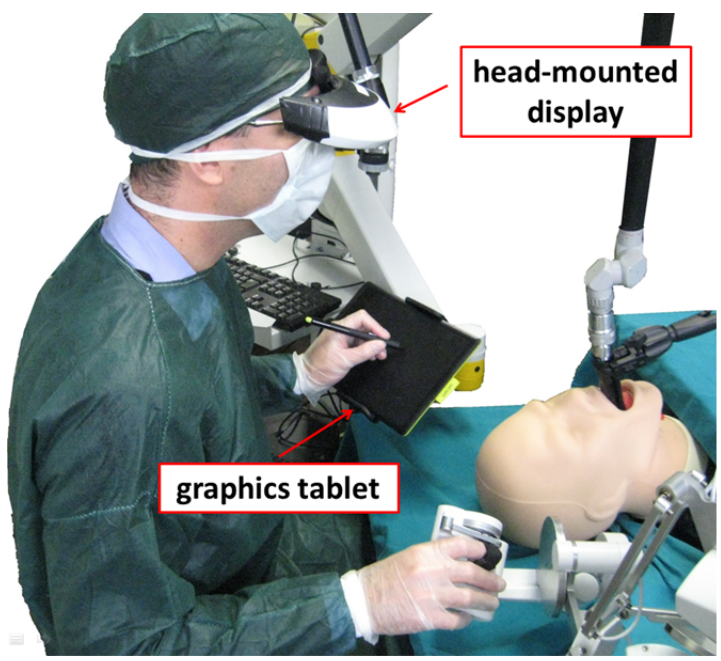

(a)

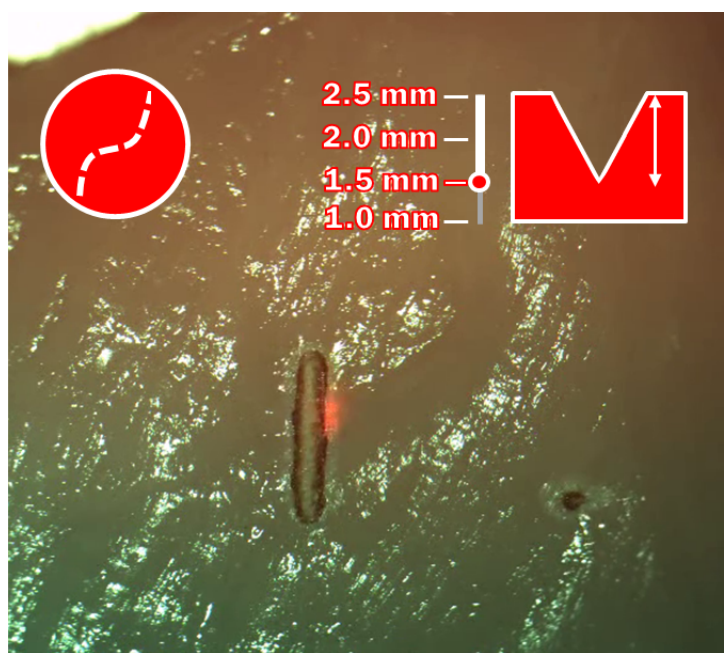

(b)

Figure 7-1: a) The $\mu$ RALP surgical interface. The surgeon visualizes the surgical site through a stereoscopic display (HMD) while controlling the position of the laser beam using a stylus and graphics tablet. b) Technology concept. A virtual control element is superimposed to the surgeon interface, enabling an intuitive control of the laser incision depth.

site.

This interface enables the intra-operative planning of laser trajectories that, once approved, are automatically executed by the system. Plans are defined and previewed in the HMD, through the superimposition of virtual overlays.

It is proposed to endow the $\mu$ RALP system with the capability of automatically performing incisions based on high level surgeon inputs, i.e., incision length, shape and depth. To this end, a new user control is added to the surgeon interface, as shown in Fig. 7-1b. This is a virtual control element, i.e., a graduated scale-bar that allows the surgeon to select the desired incision depth. With this new interface, the surgeon can pre-plan the incisions he wants to perform: the incision length and shape are defined using the graphics tablet, while the incision depth is regulated through the new virtual control element.

The proposed technology relies on the availability of a controller capable of mapping the desired incision depth to appropriate laser parameters and activation. In a previous work, it has been explored how the automatic activation/deactivation of the 
laser can be utilized to realize such a control [90]. The method uses a feed-forward controller based on a laser-tissue interaction model extracted from experimental data. Here, that work was extended by incorporating the laser power and motion in the controller. A two-step approach is used: the relation between the laser parameters and the theoretical spatial distribution of energy delivered to the tissue is investigated. Then, the relation between the spatial distribution of energy and the resulting laser incision depth is studied.

\subsection{Modeling the Laser Incision Process}

In current surgical practice, incisions are produced by repeatedly moving the laser beam along desired cutting paths on the tissue. Laser scanning uses motorized mirrors to deflect the laser beam, enabling the automatic execution of preprogrammed cutting patterns. High-frequency cycles of the laser motion across the target tissue remove overlying layers with each pass.

$\mathrm{CO}_{2}$ laser sources used in TLM present an emission in the infrared range (wavelength: $10.6 \mu \mathrm{m}$ ), which is strongly absorbed by the water content of the tissue [93]. This induces a thermal interaction that results in the vaporization of tissue. Ablation by vaporization is the fundamental physical process that enables the creation of surgical laser incisions. The resulting ablation crater presents the same shape of the laser beam [86].

\subsubsection{Spatial energy distribution}

Let us consider a small piece of tissue exposed to a laser beam. For the sake of simplicity, it was assumed the surface of the tissue to be flat. It was also assumed that the laser is firing uninterruptedly on the tissue, i.e., it is operating in continuous wave $(\mathrm{CW})$ mode. A movement of the laser on the surface can be described as a curved area $\gamma$ in $\mathbb{R}^{2}$, with endpoints $a$ and $b$ (see Fig. 7-2). From a fundamental physical law, considering $d s$ is an infinitesimal increment along $\gamma$ and $d r$ an infinitesimal increment along the laser beam cross-section profile, each infinitesimal area $d A=d s \cdot d r$ receives 
an energy dose equal to the laser output power $P$ times the duration of laser exposure:

$$
\partial E=P \partial t
$$

where $E[\mathrm{~J}]$ is the total amount of energy delivered by the laser during its motion along $\gamma$. (7.1) implies that the distribution of laser energy depends on the exposure time when using a constant power level $(P)$ : shorter exposure results in smaller delivered energy compared to longer exposures. This implies that the distribution of laser energy along the sweeping path depends on the speed of the laser motion: faster movements determine shorter exposures and, thus, smaller amount of energy; similarly, slower movements results in higher amounts of energy. The energy density $e(A)\left[\mathrm{J} / \mathrm{m}^{2}\right]$ is defined as the derivative of the energy $E$ along $\gamma$;

$$
e(A)=\frac{\partial E}{\partial r \partial s} n
$$

where $n \in \mathbb{N}$ represents the number of repeated passes (laser scans) performed on the tissue. Replacing the term $E$ from (7.1) in (7.2) yields,

$$
e(A)=\frac{P}{\partial r} \frac{\partial t}{\partial s} n
$$

This equation establishes a relation between the energy density $e(A)$ and the speed of laser motion: these two quantities are inversely proportional, with $n P$ being the proportionality factor. Such a relation can be used to control the distribution of energy along the incision path, through the regulation of the laser power, speed of motion, and number of passes.

It is assumed that the laser is perfectly focused on the target tissue, which provides a constant laser spot diameter, $w$. With this assumption, (7.3) can be simplified to:

$$
e(A)=\frac{P}{w} \frac{\partial t}{\partial s} n
$$

Now, the relation between the laser energy density $e(A)$ and the resulting incision depth $d(A)$ will be considered. Prior research has shown that the latter increases 


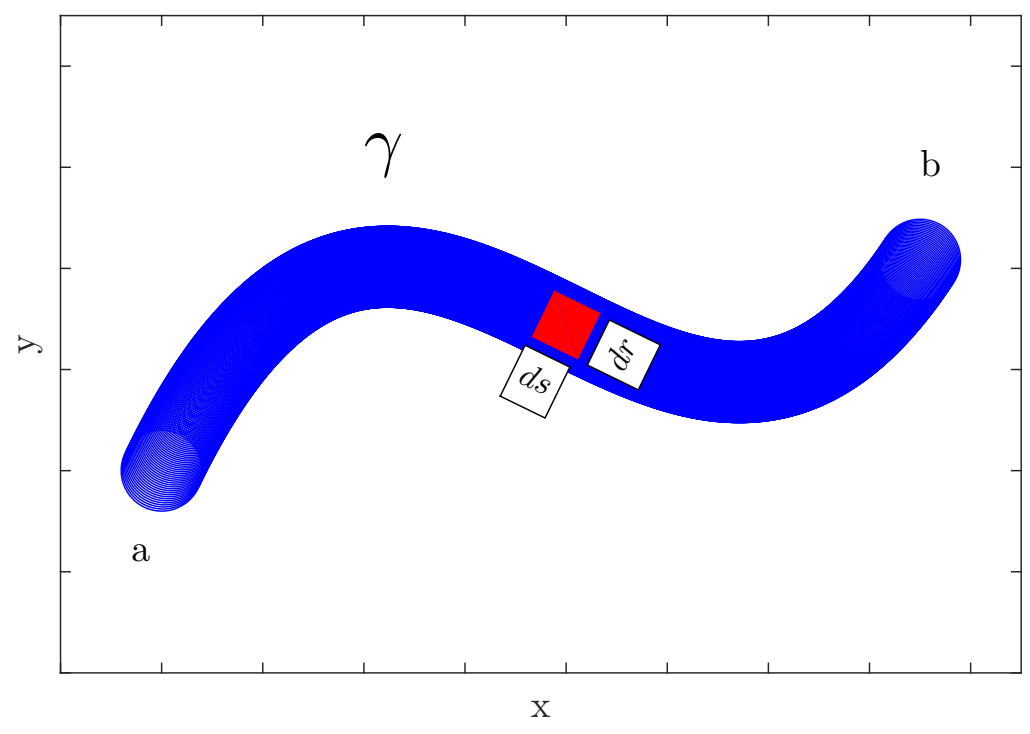

Figure 7-2: The motion of a the laser from a point $a$ to $b$ can be represented as a curve $\gamma \in \mathbb{R}^{2}$.

linearly with the total time of laser exposure, all other parameters being fixed $[90,94]$. Based on these evidence, here, it is hypothesized that a similar relation exists between $e(A)$ and $d(A)$, i.e.,

$$
d(A)=\alpha \cdot e(A)+\beta
$$

where $\alpha, \beta \in \mathbb{R}^{+}$in $\mathrm{mm}^{3} / \mathrm{J}$.

\subsubsection{Feed-forward controller}

In order to derive the feed-forward controller, the relation between energy density, $e$, the number of laser passes, $n$, and the incision depth, $d$, is investigated.

$$
d=f(e, n)
$$

Given the desired incision depth, $d_{t}$, this controller calculates the required number of passes, $n$, and required energy density, $e_{r e q}$ (Fig. 7-3). 


\subsection{Methodology}

\subsubsection{Experiments}

This section describes the experiments conducted to test the validity of the hypothesis formulated in the last section. Based on (7.4), $e(A)$ can be controlled through the manipulation of three distinct inputs, i.e., the laser power $P$, the speed of motion $\partial s / \partial t$, and the total number of laser passes $n$. It should be noted that the same energy density can be delivered to the tissue using different combinations of these three inputs. Therefore, it was first investigated whether the depth of incision depends solely on the energy density, or the specific combination of laser inputs. Later on, it was reported on an experiment aimed at understanding the feasibility of controlling the laser incision depth automatically. In all experiments, it is assumed that laser spot is focused perfectly with $250 \mu \mathrm{m}$ diameter $(w=250 \mu \mathrm{m})$.

\section{Single-pass experiment}

An experiment is performed to compare the effects of creating the same energy distribution $e(A)$ through different combinations of laser power and speed. To exclude the effect of repeated laser passes on the resulting incision depth, single-pass incisions were performed, i.e., $n=1$. The experimental design involves three pairs of conditions: for each pair, the amount of energy involved was fixed. The assigned values were $e(A)=[1.2 ; 2.4 ; 3.6] \mathrm{J} / \mathrm{mm}^{2}$. These have been selected in order to cover the typical range of energy used during real laser microsurgeries. Each of these experimental conditions was implemented using two different combinations of laser power

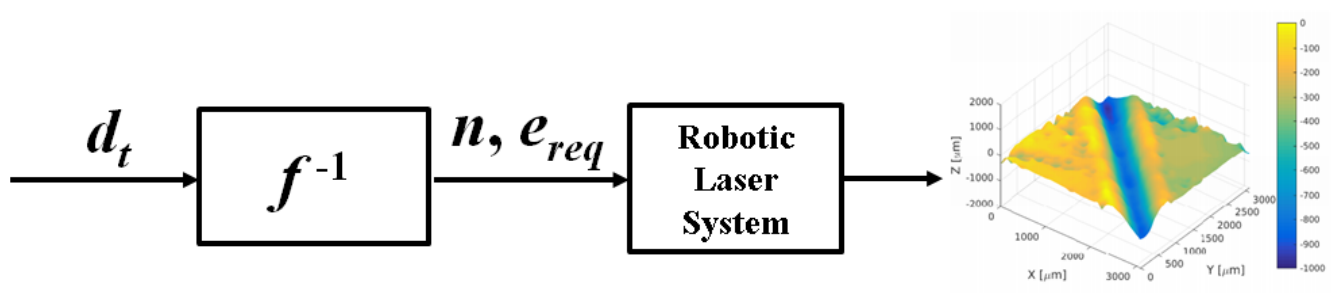

Figure 7-3: The feed-forward controller scheme. 
and incision speed. Three repetitions were performed for each configuration, resulting in a total of eighteen incision trials.

\section{Multi-pass experiment}

The focus of this experiment is on understanding the effect of repeated laser passes, performed at constant speed and with fixed laser power, on the resulting incision depth. To this aim, a set of incision trials was performed each time increasing number of passes, i.e., $n=[1 ; 2 ; 4 ; 6]$. The same set was repeated with two different combinations of laser power, i.e., $P=[4 ; 8] \mathrm{W}$. Speed was set to $\partial s / \partial t=[13.3 ; 26.7]$ $\mathrm{mm} / \mathrm{s}$, respectively. Each combination is repeated three times, resulting in a total of 24 incision trials.

\section{Computer-controlled laser incision trials}

The aim of this experiment is to understand the feasibility of creating specific target incision depths by controlling the energy density $e(A)$ created by the laser system. The relation between these two quantities is established through a regression method based on the data collected during the previous two experiments. Here, the inverse of such relation is used to drive the selection of the number of laser passes and energy density required to realize the target depth.

The experiment involves three different target depths, i.e., $d=[0.3 ; 0.5 ; 0.8] \mathrm{mm}$; for each target depth, three repetitions were performed, resulting in a total of nine trials. The laser power and speed are fixed to $P=4 \mathrm{~W}$ and $\partial s / \partial t=[11.0 ; 10.1 ; 8.7]$ $\mathrm{mm} / \mathrm{s}$, respectively. The selection of the number of laser passes $n$ is performed using a procedure that limits the energy delivered in the course of a single pass. The motivation for using such an approach is that higher energy densities are known to induce stronger thermal interactions, thereby resulting in deformations of tissue that may hinder the repeatability of the trials. This effect was noticeable also in the results of the previous experiments, as will be shown later.

The automatic procedure for computing the necessary number of laser passes and energy density to achieve a target incision depth is described as follows. First, the en- 


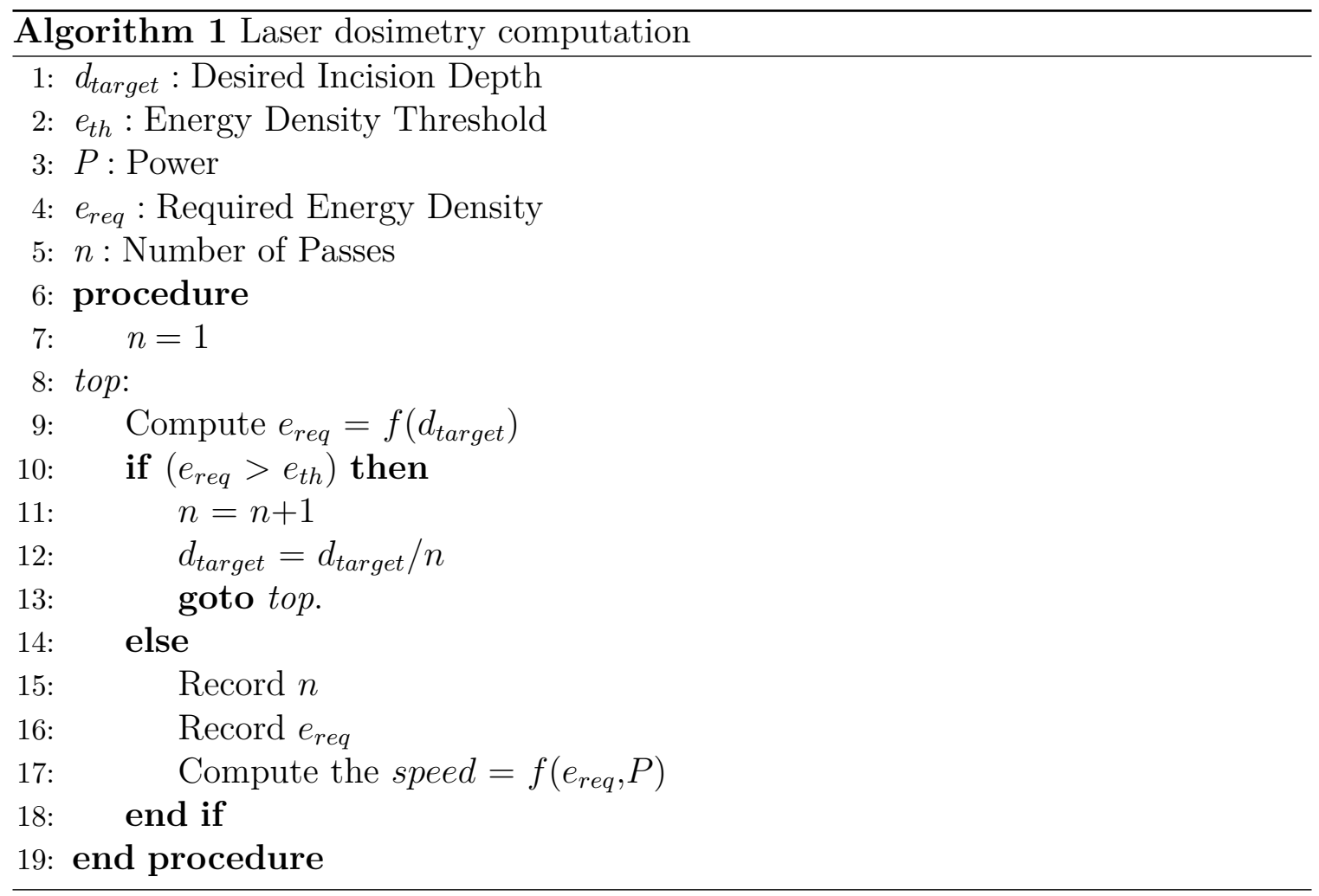

ergy density $e(A)$ that would be required to reach the target depth using a single pass (i.e., $n=1$ ) is computed. This is calculated based on laser-tissue interaction model learned for data gathered in the course of previous experiments. If the energy density value exceeds the threshold of $2 \mathrm{~J} / \mathrm{mm}^{2}$, then the number of passes is incremented. This procedure is repeated until such condition is met. Algorithm 1 summarizes this laser dosimetry computation procedure.

The specific threshold value used was selected after analyzing the results from previous experiments, which indicated that energy densities smaller than this value result in more repeatable incision depth results. Three incisions were performed for each configuration, resulting in a total of 9 incisions. For each incision, 20 measurements were performed as described in the following section.

\subsubsection{Materials and measurements}

In this section, the materials used in the experiments and measurement methods employed for the evaluation of the results are explained. 


\section{Laser source}

The experimental setup uses a commercial surgical laser source, a DEKA SmartXide ${ }^{2}$ $\mathrm{CO}_{2}$ (wavelength $10.6 \mu \mathrm{m}, \mathrm{TEM}_{00}$ beam profile). The HiScan Surgical laser scanner is used to regulate the speed of the laser spot on the tissue. Incision profile is a $5 \mathrm{~mm}$ straight line defined with the interface of the system in all experiments.

\section{Soft tissue samples}

Ex vivo chicken muscle tissue was used in these experiments. This type of soft tissue has a high water content, which makes it a suitable target for $\mathrm{CO}_{2}$ laser ablation trials. Before the experiments, tissue samples were kept for 10 minutes in an open refrigerated box at a controlled temperature $\left(7-12^{\circ} \mathrm{C}\right)$, in order to preserve their moisture and prevent degradation. To ensure identical initial temperature, the samples were monitored with an infrared thermal camera. Different tissue samples were used for each experimental condition, adding to the experiments a random effect. It is important to point out that the tissue model selected in this study may not present the same laser absorption profile that would be found in tissues in vivo conditions, since the thermal effects of a laser on living tissues are influenced by factors that are not present in ex vivo models [93], e.g., convective heat transfer due to blood perfusion. Nonetheless, most of these factors can be neglected in a first approximation [93] for the purpose of this proof-of-concept study. Indeed, chicken muscle tissue was selected and used for convenience since it is easily available and facilitates tissue manipulation, sample preparation and the incision depth measurements. Future research will focus on translating the modeling and control methods developed here to more realistic scenarios through trials to work out protocols on ex vivo pig larynxes and in vivo animal tissues.

\section{Microscope examination}

For estimating the parameters of the incision craters, confocal images (Leica SP5 microscope, Leica Microsystems GmbH, Germany) were analyzed. The resolution 


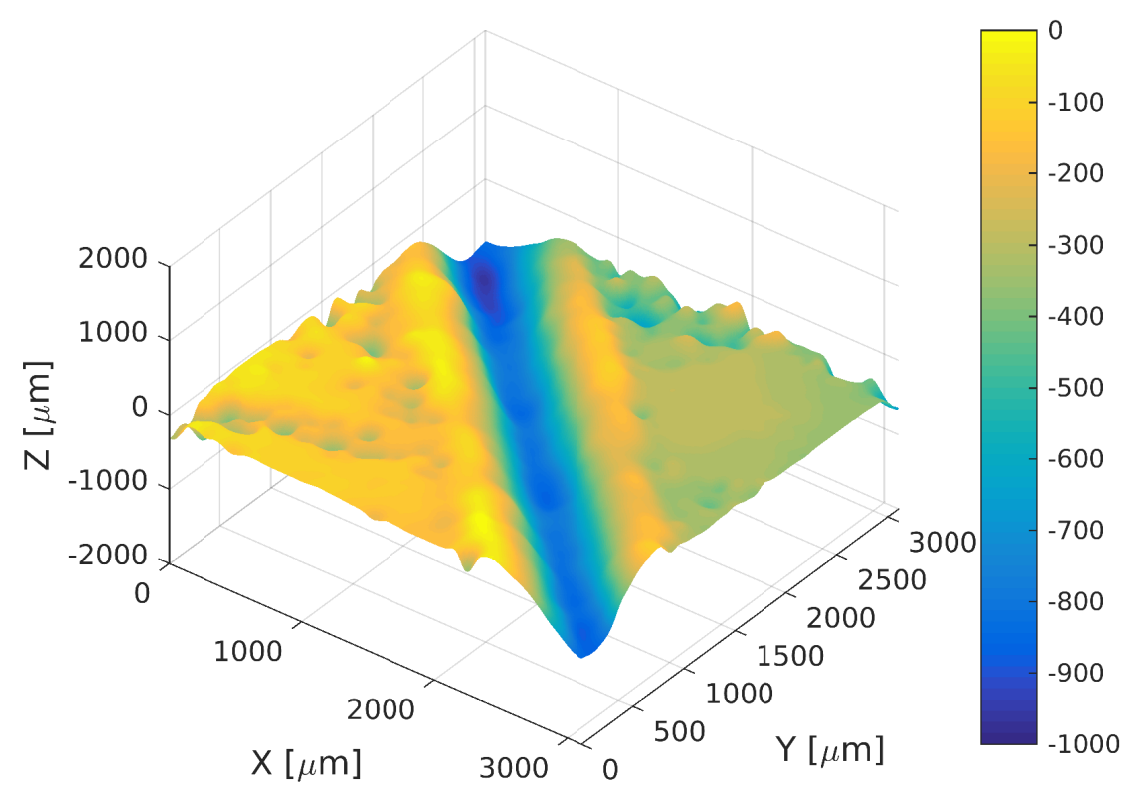

Figure 7-4: Example of a depth map showing an incision crater produced on top of a tissue sample. This map has been reconstructed from microscopic images using the algorithm described by Aguet et al. Colorbar is in $\mu \mathrm{m}$.

of the images had to be compromised so as to accommodate the dimension of the specimen using a 5x/0.15 numerical aperture (NA) air objective. The images were acquired using an upright configuration in reflection mode with emission line centered at $488 \mathrm{~nm}$. This optical configuration enables the analysis of area up to $3.1 \times 3.1 \mathrm{~mm}^{2}$. Preliminary trials revealed that a lateral (XY) resolution of $6 \mu \mathrm{m}$ per pixel and an axial (Z) resolution of $30 \mu \mathrm{m}$ provide an adequate trade-off between the level of image detail and the acquisition time. The lateral and axial resolutions can be regulated by the user using objectives with different NA and laser lines.

Microscopic images have been processed with an extended depth of field algorithm [74], that produces a depth map representing the three-dimensional topology of the sample surface (see Fig. 7-4).

\section{Measurement of the incision depth}

An example of incision profile is shown in Fig. 7-5. A two-term Gaussian fitting procedure is used to approximate the lateral profile of the incision. Boundaries are 


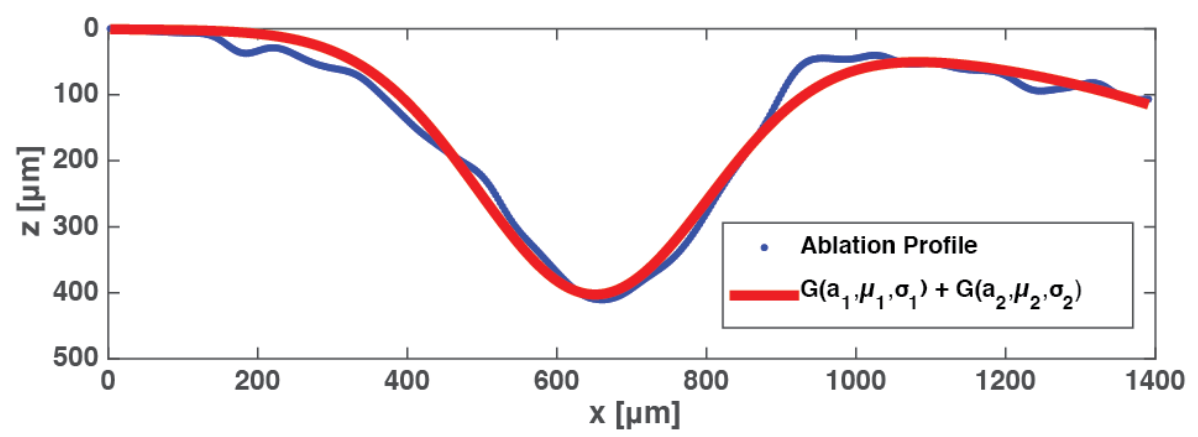

Figure 7-5: Example of Gaussian fitting. Estimated parameters are amplitude (a), mean $(\mu)$, standard deviation $(\sigma)$. For this particular example, $a_{1}=392.7, \mu_{1}=$ 649.7, $\sigma_{1}=219.1, a_{2}=2 \cdot 10^{6}, \mu_{2}=7649, \sigma_{2}=2000$. The parameter $a_{1}$ is taken as the depth of the crater.

imposed on the fitting problem, so that (i) one-term models the altitude and profile of the tissue surface, while (ii) the second term models the profile of the ablation crater produced by the laser. The amplitude of this latter term is taken as measure of the laser incision depth. The profile of each incision crater is sampled at intervals along its central part. Sampling interval is $60 \mu \mathrm{m}$, resulting in 20 profiles for each incision trial.

\subsection{Results}

\subsubsection{Single-pass experiment}

The plot in Fig. 7-6 summarizes the results for each of the six experimental configurations. The choice of a higher laser power level was found to produce deeper incisions, the energy density $e(A)$ being equal. A Kruskal Wallis test was performed to support that measurement results for each pair of $4 \mathrm{~W}$ and $8 \mathrm{~W}$ are not overlapping; the results of the test show that the null hypothesis was rejected with the following $p$ values, $1.8 e^{-9}, 1.2 e^{-12}$ and $3.4 e^{-10}$ for $1.2,2.4$ and $3.6 \mathrm{~J} / \mathrm{mm}^{2}$, respectively. Table 7.1 reports the mean and spread (standard deviation) observed for each experimental configuration. The difference in the mean depth was found to increase for different power levels as the applied energy is increased (Fig. 7-6). 


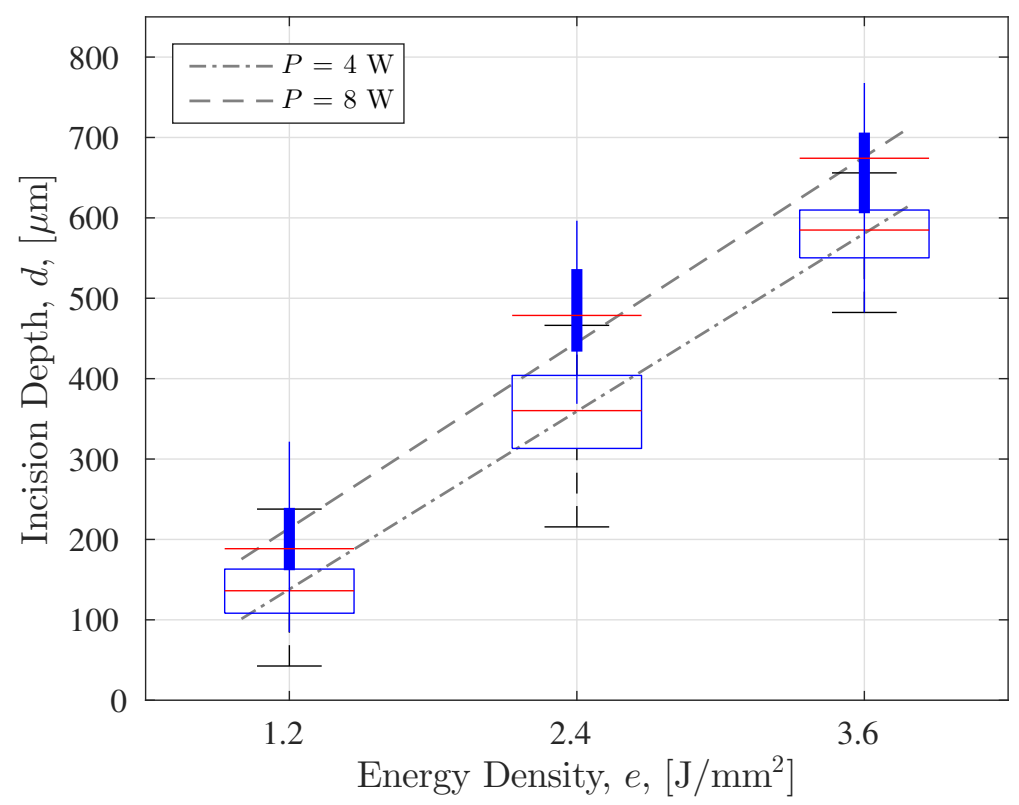

Figure 7-6: Incision depth, $d[\mu \mathrm{m}]$, produced with different combination of laser power and speed in chicken muscle tissue (see Table 7.1). Results obtained with laser power $P=4 \mathrm{~W}$ are represented by empty box plots, while those for $P=8 \mathrm{~W}$ are represented by filled box plots. For a given value of laser power, the incision depth depends linearly on the energy density (for $4 \mathrm{~W}, d=181.2 \cdot e-73.8$ and for $8 \mathrm{~W}, d=211.9 \cdot e-48.7$ ).

For each laser power value considered, the relation between the energy density and the incision depth was found to be linear, with a fitting root mean squared error (RMSE) of $2.1 \mu \mathrm{m}$ for $4 \mathrm{~W}$, and $27.1 \mu \mathrm{m}$ for $8 \mathrm{~W}$.

\subsubsection{Multi-pass experiment}

The plots in Fig. 7-7 show the relative depth increment $\Delta d=d / \overline{d_{s}}$, where $\overline{d_{s}}$ is defined as the mean incision depth created with a single laser pass (i.e., $n=1$ ). In the experimental scenario with laser power $P=4 \mathrm{~W}$, the mean values of $\Delta d$ were 1.9, 4.0 and 5.5 for 2,4 and 6 passes, respectively. A linear regression $\Delta d=\alpha n+\beta$ with parameters $\alpha=0.9, \beta=0.14$ approximates these points with $\mathrm{RMSE}=0.13$. A smaller increment rate was observed for $P=8 \mathrm{~W}(\alpha=0.76, \beta=0.3)$. The approximation error obtained in the scenario was higher with respect to the lowerpower configuration, i.e., RMSE $=0.28$. This can be largely attributed to the results 
Table 7.1: Variance of incision depth produced with different combinations of laser power and speed

\begin{tabular}{|c|c|c|c|c|}
\hline $\begin{array}{l}\mathrm{e}(\mathrm{A}) \\
\mathrm{J} / \mathrm{mm}^{2}\end{array}$ & Power & Speed & $\begin{array}{c}\text { Mean } \\
\text { Depth } \\
\mu \mathrm{m}\end{array}$ & $\begin{array}{c}\text { Standard } \\
\text { Deviation } \\
\mu \mathrm{m}\end{array}$ \\
\hline \multirow{2}{*}{1.2} & 4 & 13.3 & 143 & 39.6 \\
\hline & 8 & 26.7 & 194 & 53.0 \\
\hline \multirow{2}{*}{2.4} & 4 & 6.7 & 362 & 57.9 \\
\hline & 8 & 13.3 & 483 & 64.7 \\
\hline \multirow{2}{*}{3.6} & 4 & 4.4 & 578 & 40.9 \\
\hline & 8 & 8.9 & 703 & 67.8 \\
\hline
\end{tabular}

obtained with higher number of passes $(n=[4 ; 6])$, that, as can be seen from Fig. 77(b), present a deviation from a linear behavior. Mean relative increments observed for this scenario are 1.7, 3.8 and 4.6 for 2,4 and 6 passes, respectively.

\subsubsection{Computer-controlled laser incisions}

Computer-controlled laser incisions were performed considering the target depths presented in Table 7.2. For each case, the laser dosimetry parameters were computed using the linear regression model presented in Fig. 7-8a and Algorithm 1, which defined both the number of laser passes and the energy density values to be used to achieve the desired incision depths. The table also reports the mean and standard deviation for each experimental condition. The measured incision depths can be compared visually against their targets in Fig. 7-8b. Deviations from the target are $4.7 \%, 5.8 \%$ and $4.9 \%$ for 300, 500 and $800 \mu \mathrm{m}$, respectively. Fig. 7-9 shows a sample incision depth map for each of the three experimental conditions.

The residual error plot for computer-controlled incisions is depicted in Fig. 7-10. The results show that the residual errors are concentrated around zero with a tendency to negative values indicating that the incisions tented to be slightly shallower than the commanded values. 
a) $P=4 \mathrm{~W}$

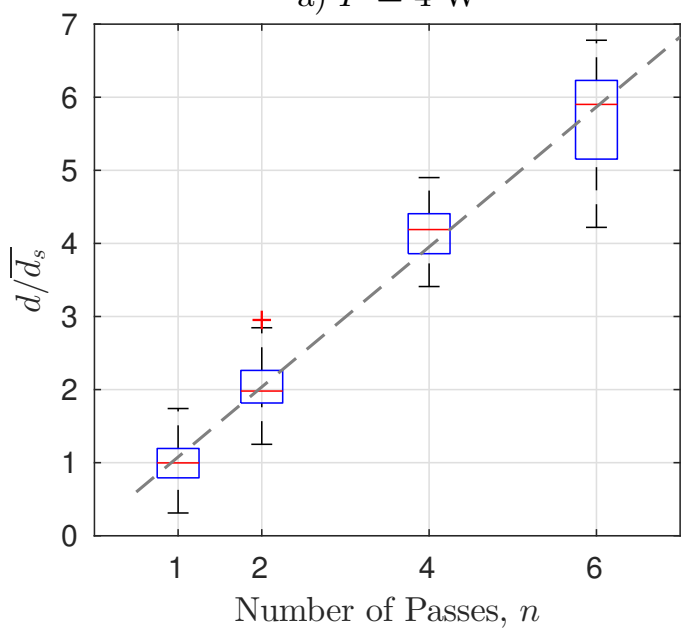

b) $P=8 \mathrm{~W}$

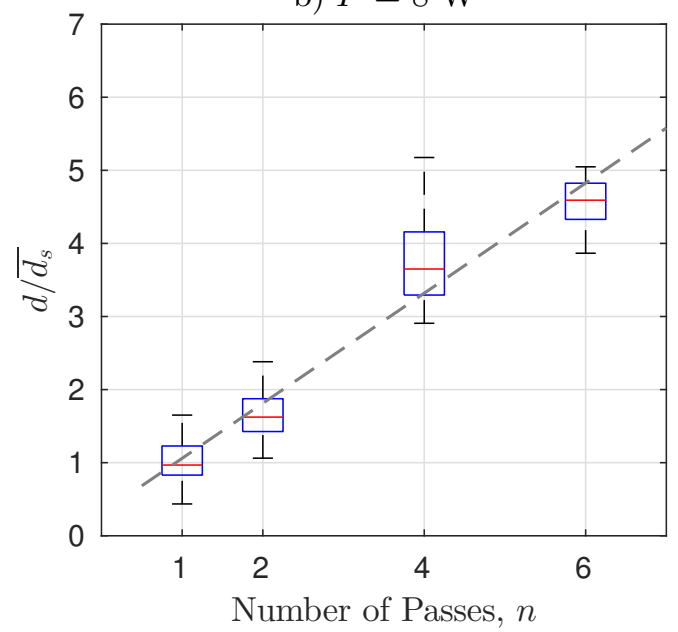

Figure 7-7: Relative incision depth, $d / \overline{d_{s}}$ for different number of passes. $\overline{d_{s}}$ is average depth of the incisions produced with single pass. For all configurations, energy density, $e$, is fixed to $1.2 \mathrm{~J} / \mathrm{mm}^{2}$ and data are presented for $P=4 \mathrm{~W}-\overline{d_{s}}=143 \mu \mathrm{m}$ (a), and $P=8 \mathrm{~W}-\overline{d_{s}}=194 \mu \mathrm{m}(\mathrm{b})$.

a) $P=4 \mathrm{~W}$

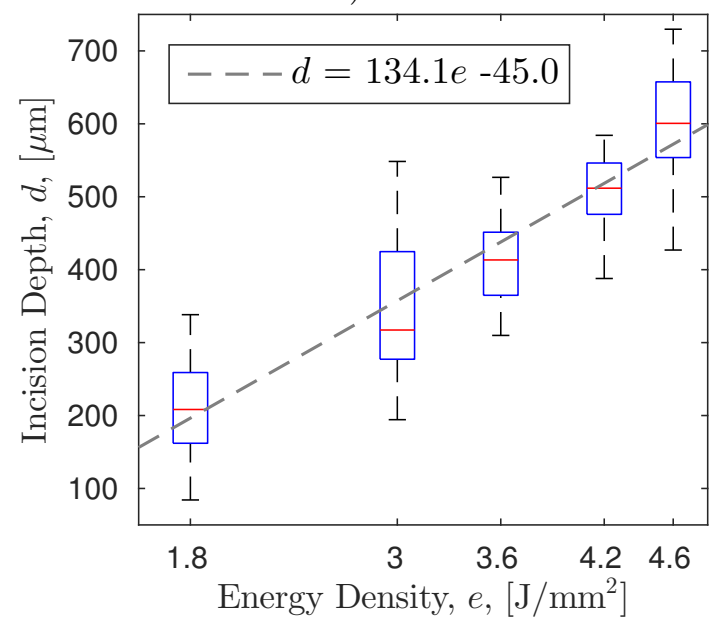

b) $P=4 \mathrm{~W}$

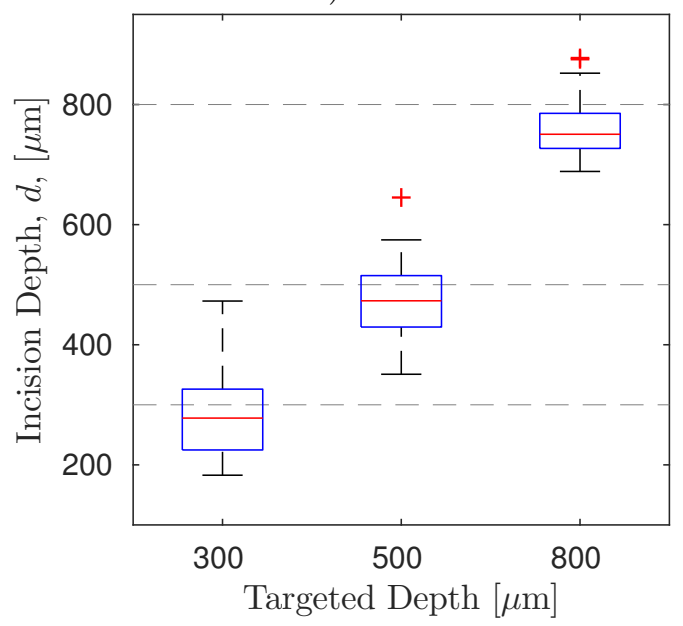

Figure 7-8: a) Incision depth, $d[\mu \mathrm{m}]$, for different energy densities, $e\left[\mathrm{~J} / \mathrm{mm}^{2}\right]$, and linear regression used to calculate the parameters for targeted depths. b) Incision depth, $d[\mu \mathrm{m}]$, for targeted depths $[\mu \mathrm{m}]$. 
Table 7.2: Results of the Controlled Incision Trials

\begin{tabular}{lccccc}
\hline Target & $\mathbf{n}$ & $\mathbf{e}(\mathrm{A})$ & Speed & $\begin{array}{c}\text { Mean } \\
\text { Depth }\end{array}$ & $\begin{array}{c}\text { Standard } \\
\text { Deviation }\end{array}$ \\
$\mu \mathrm{m}$ & & $\mathrm{J} / \mathrm{mm}^{2}$ & $\mathrm{~mm} / \mathrm{s}$ & $\mu \mathrm{m}$ & $\mu \mathrm{m}$ \\
\hline 300 & 2 & 1.46 & 11.0 & 286 & 70 \\
500 & 3 & 1.58 & 10.1 & 471 & 55 \\
800 & 4 & 1.83 & 8.7 & 761 & 47 \\
\hline
\end{tabular}

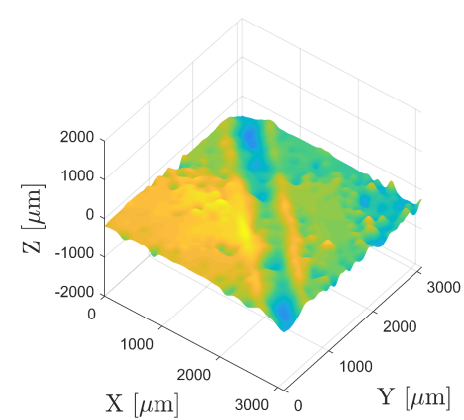

(a) $300 \mu \mathrm{m}$

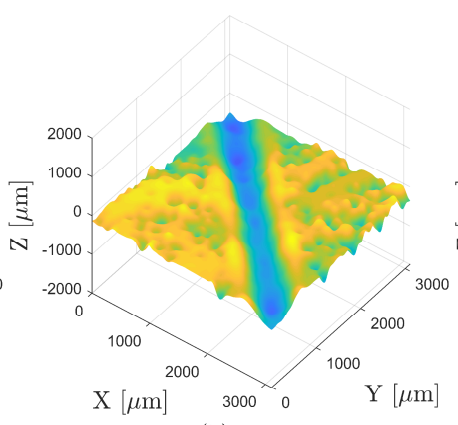

(b) $500 \mu \mathrm{m}$

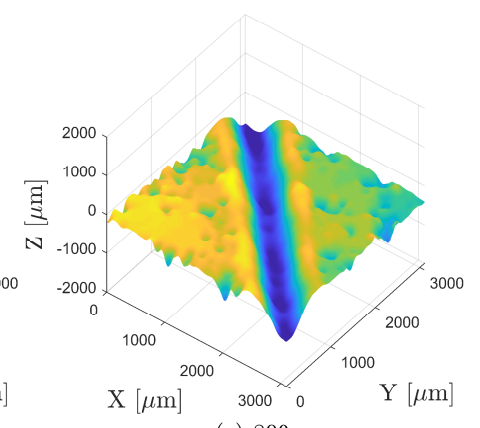

(c) $800 \mu \mathrm{m}$

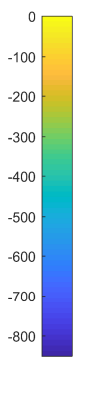

Figure 7-9: Comparison of depth maps of incisions performed with different energy densities and number of passes for targeted values; a) $300 \mu \mathrm{m}-\left[e=1.46 \mathrm{~J} / \mathrm{mm}^{2}, v\right.$ $=11.0 \mathrm{~mm} / \mathrm{s}, n=2]$, b) $500 \mu \mathrm{m}-\left[e=1.58 \mathrm{~J} / \mathrm{mm}^{2}, v=10.1 \mathrm{~mm} / \mathrm{s}, n=3\right]$, c) 800 $\mu \mathrm{m}-\left[e=1.83 \mathrm{~J} / \mathrm{mm}^{2}, v=8.7 \mathrm{~mm} / \mathrm{s}, n=4\right]$. Colorbar is in $\mu \mathrm{m}$.

\subsection{Discussion}

Results of the single-pass experiments indicate that knowledge of the laser energy density alone is not sufficient to predict the laser incision depth: the laser power must be also taken into account. In the literature, laser incision depth has been considered as a simple function of the total amount of energy delivered by laser [22]. However, results demonstrated that incision depth increases as the power is increased for the same energy density level.

The relation between incision depth and energy density is linear, when the power of the laser is fixed, as postulated in (7.5). This result is consistent with previous works $[90,94]$ where the authors reported a linear relation between exposure time and incision depth.

Results of the multi-pass experiment present linear behavior for the relation be- 


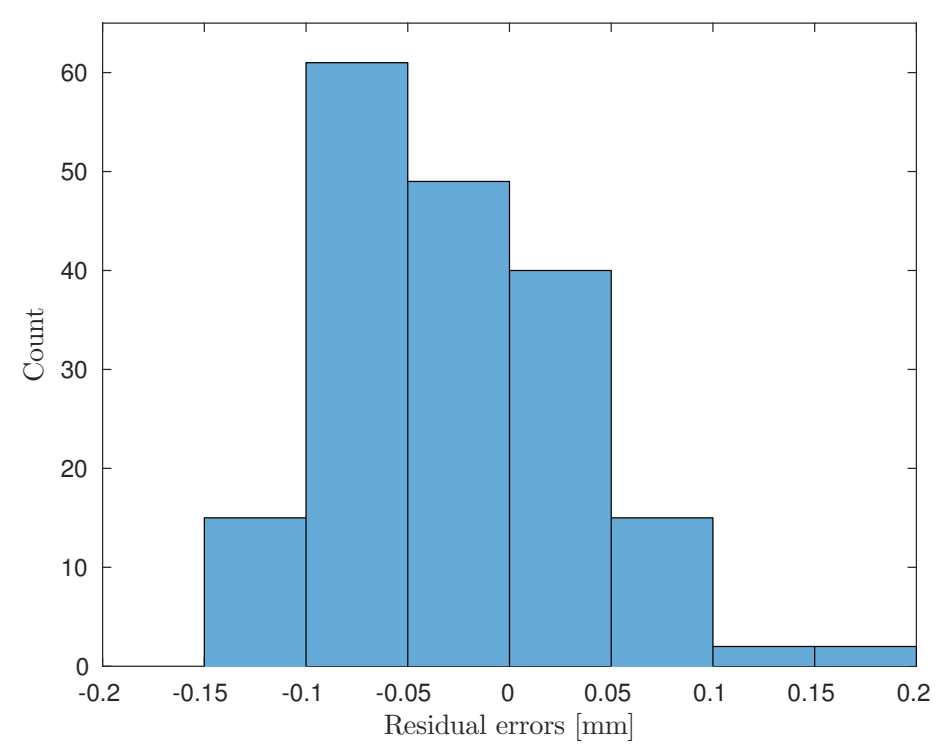

Figure 7-10: Histogram plot showing residual errors for the computer-controlled incision trials.

tween relative incision depth and number of passes. In addition to this, multi-pass experiments results show that such relationship holds for incisions that are not more than $916 \mu \mathrm{m}$. In a previous study where researchers performed multi-pulse laser incision experiment on bone tissue [85], it was reported that the number of pulse depth relation is linear up to $1 \mathrm{~mm}$ depth whereas logarithmic relation exists up to $2 \mathrm{~mm}$. As in that case, also here it was observed that when incisions are performed with several passes, as the incision goes deeper, energy dissipation is observed due to changing focus of the laser spot, incision debris and increasing the surface area of incision [85].

Multi-pass experiment results for $4 \mathrm{~W}$ show that relative incision depths, $\Delta d$, for all data points are close to unity. Increasing the power to $8 \mathrm{~W}$ causes the relationship to deviate from being linear. In this set of experiments, incisions are performed with higher speed and higher power. A possible reason for deviation from linearity can be higher thermal deformations at high power levels. It is also reported that temperature increases start earlier at higher power [95]. Authors also reported that instant temperature rises up to $20^{\circ} \mathrm{C}$ are observed for the incisions produced with $12 \mathrm{~W}$. There is not sufficient time for thermal relaxation with the incisions in the 
continuous wave mode in contrary to pulsed laser incisions. Thus, the remaining heat after vaporization of the tissue is dissipated to neighboring tissue, causing also the ablation of the these structures when working at higher power levels [95].

For computer-controlled incisions, $4 \mathrm{~W}$ was selected due to the low standard deviation in single-pass experiments and highly linear behavior in multi-pass experiments. Computer-controlled incision results indicate that targeted depth can be achieved within $\pm 100 \mu \mathrm{m}$ error range. However, as Table 7.2 shows, mean depth errors were only 14, 29 and $39 \mu \mathrm{m}$ for 300, 500 and $800 \mu \mathrm{m}$ targeted depths, respectively. Highest mean error was observed for $800 \mu \mathrm{m}$ targeted depth, which was performed with 4 passes, i.e., $n=4$. As discussed earlier, as the number of passes increases in multi-pass incisions, the resulting incision depth tends to deviate from linear behavior due to the energy dissipation. Nevertheless, the measured standard deviations were significantly small: 70, 55 and $47 \mu \mathrm{m}$ for 300, 500 and $800 \mu \mathrm{m}$, respectively. This indicates the proposed technology provides repeatable results.

\subsection{Conclusion}

In this chapter, the concept of a technology to automate laser incisions on soft tissue was presented for laser microsurgery applications. An existing robotic laser device is used to realize the laser motion on the surgical site. A feed-forward controller maps high-level commands imparted by the surgeon to the laser parameters required to achieve the desired incisions. The controller is based on an inverse model, which is extracted from experimental data using regression techniques.

Experimental evidence presented here indicates that the depth of a laser incision can be regulated controlling the energy density along the incision path and number of passes with an accuracy of $\pm 100 \mu \mathrm{m}$. These results provide data on the methodological concept for an extended protocol for the implementation and validation of an improved robot-assisted technology in real laser microsurgery conditions in clinical setting. However, a clinical implementation of this new technology will still require the use of the proposed method for the derivation of new models considering the same 
type of living tissues encountered during real laser microsurgeries. This is required because the properties of the living tissues, such as the water and blood content, vary not only between different tissue types, but also significantly from ex vivo conditions [96]. Therefore, in order to validate the proposed system and bring it closer to clinical application in the operating room, it is crucial to extend this work with in vivo experiments. Enough data will have to be collected to create models that generalize well and allow good incision depth control by applying the same methodology presented here.

Given that at the present time no technology is available to control or supervise the creation of laser incisions during laser microsurgeries, the proposed technology represents a significant advance to the state-of-the-art technology for laser microsurgery. It has the potential to facilitate and significantly enhance the surgeon's capacity to create precise laser incisions, allowing clinical reasoning based on more intuitive quantities - i.e., the incision depth - and leaving to the robotic system the task of regulating the energy delivered to achieve the desired results. In the future, it will be also explored the integration of the haptic feedback into the models developed here. A previous research demonstrated that introducing the haptic feedback into the laser microsurgery workflow allows significant improvements in performance in laser incision depth control [97]. 



\section{Chapter 8}

\section{Discussion and Conclusion}

\subsection{Discussion}

The total workspace of the prototype magnetic laser scanner is $4 \times 4 \mathrm{~mm}^{2}$. Prior research indicates that surgeons prefer the high-speed scanning lengths in the range of 1-2 mm [23, 24, 98]. However, commercial systems with mirror-based scanning provide incision lengths up to $5 \mathrm{~mm}$ [24]. Therefore, the achieved total workspace is comparable to the state-of-the-art systems and to the needs of surgeons. Nonetheless, it is worth noting that the workspace of the magnetic laser scanner can be further increased by adapting the optical design for longer working distances between the target and the tip of the scanner. The extent of the workspace would increase linearly with the increasing focal length. Additionally, coupling the magnetic laser scanner to the distal end of a flexible robotic endoscope would also increase the workspace by enabling the motion of the end-effector module itself. However, when adapting the optical design and integrating the system with a flexible endoscope, the total volume available at the surgical site should be considered as a design restriction.

When using the magnetic laser scanner, surgeons can define a customized trajectory with the tablet device, which is then automatically executed by the system with high accuracy and precision, allowing repeated ablations along the same path. Results from precision assessment show that repetitions of a predefined trajectory are indis-

tinguishable from each other. The measured repeatability errors are around $20 \mu \mathrm{m}$ 
for smooth trajectories and $75 \mu \mathrm{m}$ for challenging trajectories, which are comparable with the range of thermal damage caused by $\mathrm{CO}_{2}$ lasers (about $50 \mu \mathrm{m}$ ) [24].

Experimental evidence from teleoperation user trials demonstrated that compact laser micromanipulation closer to the target $(30 \mathrm{~mm})$ has a great potential to increase surgical site accessibility and control accuracy with respect to the manipulation of free-beam lasers from large distances $(400-500 \mathrm{~mm})$. In the literature, user trials with the traditional free-beam laser micromanipulator demonstrated that trajectoryfollowing errors are in between 211-650 $\mu \mathrm{m}[71,33,46]$. On the other hand, the results presented here showed that trajectory-following tasks can be accomplished close to the target with errors below $40 \mu \mathrm{m}$. The main concepts that enable this improvement are: i) decreasing the working distance, ii) providing intuitive control with a tablet device, and iii) including motion scaling and high-resolution laser micromanipulation.

The automated trajectory executions with the developed model-based feed-forward controller provides high-speed laser scanning control with $90 \mu \mathrm{m}$ accuracy at a $30 \mathrm{~mm}$ distance from the scanner tip. The errors caused by the system can be compared to the surgical resection margins and laser spot sizes for delicate microsurgeries. Surgical resection margins defines the thickness of healthy tissue on the resected malignant part. In order to ensure that malignant tissue is totally removed from the body, resection margins should be around 1-2 $\mathrm{mm}$ [79]. Thus, the automated trajectory execution errors are less than $10 \%$ of typical resection margins. In addition to this, state-of-the-art systems offer 200-250 $\mu \mathrm{m}$ laser spot diameter for delicate microsurgeries. Errors caused by the system are less than $50 \%$ of the laser spot sizes.

Possible reasons for the observed trajectory-following errors may include the manufacturing imperfections. For example, it is assumed that four identical electromagnetic coils are placed around the permanent magnet, however impedance measurements show that there 3-5\% discrepancy between them. Another assumption is that the electromagnetic coils placed in the same axis are parallel to each other. However, due to the 3D printed cylindrical structure, these electromagnetic coils may not be aligned perfectly. Finding appropriate manufacturing methods will improve the performance of the magnetic laser scanner. 
Preliminary ablation trials with the magnetic laser scanner showed that the system can focus a high-power surgical laser on a target. Plaster blocks and apple samples were ablated by coupling the magnetic laser scanner with a $1940 \mathrm{~nm}$ surgical diode laser. In these ablation trials, the distance between the scanner tip and the target was $30 \mathrm{~mm}$, highlighting that the magnetic laser scanner enables non-contact ablations. Thus, disadvantages of the fiber-based tissue ablation in contact with tissue, such as tissue sticking to the fiber, are eliminated. However, the system can perform efficient ablations only when the laser beam is focused on the target. Therefore, for precise ablations, the system should always stay in focus on the target during operations. These means that, for a clinical scenario in which tissue and surgical instrumentation move continuously, further technologies will have to be devised to keep the laser always in focus during operations.

In this magnetic laser scanner design, millimeter-size electromagnetic coils were used with a cylindrical holder close to the optical fiber with the permanent magnet. Placing electromagnetic coils close to the permanent magnet enables to actuate the system with low voltages and currents $(9 \mathrm{~V}$ and $\pm 165 \mathrm{~mA})$, which is important for the safety of the operations. However, the requirement of four electromagnetic coils within the cylindrical tool increases the external diameter of the complete system. A solution to this problem might be placing electromagnetic coils outside of the tool as in the magnetic catheter actuation mechanisms [68, 69, 70]. A permanent magnet attached optical fiber could be actuated with an external magnetic field induced by electromagnetic coils placed outside of patient. In this case, the system can be potentially minimized as small as the sizes of the permanent magnet $(1-2 \mathrm{~mm})$ that is attached to optical fiber. However, due to the larger distance between permanent magnet and electromagnetic coils, higher magnetic field strengths would be required. This could potentially limit the usage of the other metallic tools, such as surgical forceps or even endoscopic cameras, in the surgical site.

The developed algorithm to control the laser incision depth during in soft tissue microsurgeries provides $\pm 100 \mu \mathrm{m}$ accuracy. Given a target incision depth, the controller regulates the laser parameters, i.e., energy density, number of passes, and 
incision speed. This technology has a potential to facilitate and improve the capability of the surgeons to create homogeneous incision profiles at the desired depths. However, a real implementation of the proposed technology in operating room requires additional validation studies such as developing new laser-tissue interaction models for living tissues.

In this thesis, the studies were performed using the research prototype presented in Chapter 2. Progress towards human trials with the new technology will involve replacing the 3D printed structures with a sterilizable material such as stainless steel. In addition, the system should be coupled to a $\mathrm{CO}_{2}$ laser source for efficient tissue ablations considering the higher absorption coefficient at $10.6 \mu \mathrm{m}$ with respect to diode lasers. This means a design change to use a hollow-core flexible waveguide instead of the optical fiber, and also the replacement of the focusing optics with ZnSe coated lenses to optimize the transmission at the different wavelength. Furthermore, an endoscopic camera should be coupled to the system for the visualization of the surgical site. Finally, before using the system in real surgical operations, validation trials shall be performed on cadavers or animal models.

The magnetic laser scanner not only provides better accessibility to the surgical site and higher laser positioning accuracy, but also lower manufacturing costs compared to the free-beam laser scanners. In current surgical setups, only the costs of the fast steering mirror mechanism is approximately $€ 15000$. The development of an endoscopic system simplifies the manufacturing of the complete system by eliminating the requirement of fast steering mirrors, the focusing system, the beam deflection mirror, and the microscope. In the proposed system, manufacturing steps simply include producing the electromagnetic coils, machining the cylindrical structures for placing the collimating and focusing lenses, and endoscopic cameras. This reduces the total cost of the system significantly. For large-scale manufacturing of the magnetic laser scanner, standardization of the electromagnetic coil production may be challenging due to the small sizes. This problem can be solved by designing a dedicated motorized system for winding the coils. 


\subsection{Conclusion}

In this thesis, the design and control of a novel magnetic laser scanner is presented to be used in endoscopic microsurgeries. The system is designed as a tip module of a flexible robot arm in order to provide 2D position control and high-speed scanning of a surgical laser. The main contribution of this thesis is the development of a compact laser actuation mechanism to be used in endoscopic systems for improving the laser-tissue interaction. A magnetically actuated laser scanner was proposed for the laser positioning and high-speed scanning at the distal end of flexible robot arm as an alternative to the systems with piezoelectric actuation and MEMS based scanning mirrors. Different operating modes were characterized for high-speed scanning, teleoperation with a tablet device, and automated trajectory executions. A concept of technology was demonstrated for laser incision depth control in soft tissue microsurgeries with a model-based feed-forward control. The significant results presented in this thesis are summarized below.

- A magnetic laser scanner was designed and manufactured enabling 2D laser position control, high-speed laser scanning, and non-contact laser ablations.

- The total workspace of the magnetic laser scanner is $4 \times 4 \mathrm{~mm}^{2}$ achieved with a $13 \mathrm{~mm}$ proof-of-concept device.

- The magnetic laser scanner provides $75 \mu \mathrm{m}$ precision for challenging trajectories and $90 \mu \mathrm{m}$ accuracy for automated trajectory executions.

- The system enables high-speed laser actuation up to $33 \mathrm{~Hz}$ for $3 \mathrm{~dB}$ limit. With acceptable errors restricted to $\sim 1 \mathrm{mrad}(50 \mu \mathrm{m})$, any $2 \mathrm{D}$ trajectory can be executed up to $15 \mathrm{~Hz}$, which corresponds to a linear speed of $94 \mathrm{~mm} / \mathrm{s}$ at a $30 \mathrm{~mm}$ operating distance.

- Teleoperation user trials demonstrated that endoscopic laser control provides at least five times better laser positioning compared to the traditional systems with the micromanipulator. 
- The proposed laser incision depth controller is able to regulate laser parameters automatically for desired depths with an error less than $100 \mu \mathrm{m}$.

- Experimental evidence shows that during laser incision depth estimation, laser energy density is not enough: laser power must be taken into account.

\subsection{Future Research Directions}

In this thesis, a novel magnetic laser scanner has been developed for endoscopic microsurgeries. Towards to the real application in operating room, future research should focus on coupling system with a flexible robot arm, e.g., continuum robot. Complete implementation of the system will also require visualization of the surgical site with a camera or fiber bundles.

Future work shall also focus on merging the concepts presented here for endoscopic laser incision depth control with additional parameters such as laser focus, laser inclination angles, etc. Homogeneous incision profiles can be created by controlling the laser on-off times during high-speed scanning and the distance between the tip of the scanner and tissue. For controlling the distance between endoscope and tissue, motorized optical lens positioning or deformable mirrors can be used to change the working distance. In addition to this, distance measurements would be critical for the best focusing of the laser.

As far as alternative applications of the proposed technology, the magnetic laser scanner can be potentially used for optical fiber-based imaging. The system can be adapted to create scanning endoscopic optical coherence tomography (OCT) or confocal laser scanning microscopy (CLSM) for in vivo diagnosis. Intra-operative 3D imaging can be performed with the same system while performing surgeries. 3D imaging of the surgical site would assist the auto-focusing mechanism by providing distance measurements. 


\section{Bibliography}

[1] Aloysius Wehr and Uwe Lohr. Airborne laser scanning - an introduction and overview. ISPRS Journal of photogrammetry and remote sensing, 54(2):68-82, 1999.

[2] Daniel Huber, Burcu Akinci, Pingbo Tang, Antonio Adan, Brian Okorn, and Xuehan Xiong. Using laser scanners for modeling and analysis in architecture, engineering, and construction. In Information Sciences and Systems (CISS), 2010 44th Annual Conference on, pages 1-6. IEEE, 2010.

[3] Abraham Katzir. Lasers and optical fibers in medicine. Elsevier, 2012.

[4] Adam Chromy and Ludek Zalud. Robotic 3d scanner as an alternative to standard modalities of medical imaging. SpringerPlus, 3(1):13, 2014.

[5] Zahid BM Niazi, Timothy JH Essex, Remo Papini, David Scott, Neil R McLean, and Michael JM Black. New laser doppler scanner, a valuable adjunct in burn depth assessment. Burns, 19(6):485-489, 1993.

[6] Anthony J Ireland, Clare McNamara, MJ Clover, Kate House, N Wenger, Michele E Barbour, K Alemzadeh, L Zhang, and Jonathan R Sandy. 3d surface imaging in dentistry-what we are looking at. British dental journal, 205(7):387, 2008.

[7] Colin JR Sheppard. Scanning optical microscope. Electronics and Power, 26(2):166-172, 1980.

[8] Eakkachai Pengwang, Kanty Rabenorosoa, Micky Rakotondrabe, and Nicolas Andreff. Scanning micromirror platform based on mems technology for medical application. Micromachines, 7(2):24, 2016.

[9] Dvir Yelin, Imran Rizvi, WM White, JT Motz, T Hasan, BE Bouma, and GJ Tearney. Three-dimensional miniature endoscopy. Nature, 443(7113):765$765,2006$.

[10] Eric J Seibel, Richard S Johnston, and C David Melville. A full-color scanning fiber endoscope. In Proc. SPIE, volume 6083, page 608303, 2006.

[11] Jürgen Sawinski and Winfried Denk. Miniature random-access fiber scanner for in vivo multiphoton imaging. Journal of Applied Physics, 102(3):034701, 2007. 
[12] C Kumar N Patel. Continuous-wave laser action on vibrational-rotational transitions of $\mathrm{CO}_{2}$. Physical review, 136(5A):A1187, 1964.

[13] Geza J Jako. Laser surgery of the vocal cordsan experimental study with carbon dioxide lasers on dogs. The Laryngoscope, 82(12):2204-2216, 1972.

[14] M Stuart Strong. Laser excision of carcinoma of the larynx. The Laryngoscope, 85(8):1286-1289, 1975.

[15] Charles W Vaughan, M Stuart Strong, and Geza J Jako. Laryngeal carcinoma: transoral treatment utilizing the $\mathrm{CO}_{2}$ laser. The American Journal of Surgery, 136(4):490-493, 1978.

[16] Joel M White, Harold E Goodis, and Charles L Rose. Use of the pulsed nd: Yag laser for intraoral soft tissue surgery. Lasers in surgery and medicine, 11(5):455461, 1991.

[17] Emanuel M Skolnik, Lawrence Martin, King F Yee, and Michael A Wheatley. Radiation failures in cancer of the larynx. Annals of Otology, Rhinology \& Laryngology, 84(6):804-811, 1975.

[18] Marc Rubinstein and William B Armstrong. Transoral laser microsurgery for laryngeal cancer: a primer and review of laser dosimetry. Lasers in medical science, 26(1):113-124, 2011.

[19] Christopher A Milford and Paul E O'Flynn. Management of verrucous carcinoma of the larynx. Clinical Otolaryngology \& Allied Sciences, 16(2):160-162, 1991.

[20] Hans Edmund Eckel and Walter Franz Thumfart. Laser surgery for the treatment of larynx carcinomas: indications, techniques, and preliminary results. Annals of Otology, Rhinology \& Laryngology, 101(2):113-118, 1992.

[21] David A Arnold, Michael Slatkine, and Eliezer Zair. Swiftlase: a new technology for char-free ablation in rectal surgery. Journal of clinical laser medicine \& surgery, 11(6):309-311, 1993.

[22] Alfred Vogel and Vasan Venugopalan. Mechanisms of pulsed laser ablation of biological tissues. Chemical reviews, 103(2):577-644, 2003.

[23] Marc Remacle, Faridah Hassan, David Cohen, Georges Lawson, and Monique Delos. New computer-guided scanner for improving $\mathrm{CO}_{2}$ laser-assisted microincision. European Archives of Oto-Rhino-Laryngology and Head \& Neck, 262(2):113119, 2005.

[24] Marc Remacle, Georges Lawson, Marie-Cècile Nollevaux, and Monique Delos. Current state of scanning micromanipulator applications with the carbon dioxide laser. Annals of Otology, Rhinology \&6 Laryngology, 117(4):239-244, 2008. 
[25] Onur Ferhanoglu, Murat Yildirim, Kaushik Subramanian, and Adela Ben-Yakar. A 5-mm piezo-scanning fiber device for high speed ultrafast laser microsurgery. Biomedical optics express, 5(7):2023-2036, 2014.

[26] Kevin K Tsia, Keisuke Goda, Dale Capewell, and Bahram Jalali. Simultaneous mechanical-scan-free confocal microscopy and laser microsurgery. Optics letters, 34(14):2099-2101, 2009.

[27] Alperen Acemoglu and Leonardo S Mattos. Magnetic laser scanner for endoscopic microsurgery. In Robotics and Automation (ICRA), 2017 IEEE International Conference on, pages 4215-4220. IEEE, 2017.

[28] C Arturo Solares and Marshall Strome. Transoral robot-assisted $\mathrm{CO}_{2}$ laser supraglottic laryngectomy: Experimental and clinical data. The laryngoscope, 117(5):817-820, 2007.

[29] Shaun C Desai, Chih-Kwang Sung, David W Jang, and Eric M Genden. Transoral robotic surgery using a carbon dioxide flexible laser for tumors of the upper aerodigestive tract. The Laryngoscope, 118(12):2187-2189, 2008.

[30] Amelia P Bailey, Luca Lancerotto, Chad Gridley, Dennis P Orgill, Hiep Nguyen, Elena Pescarini, Gianluigi Lago, and Antonio R Gargiulo. Greater surgical precision of a flexible carbon dioxide laser fiber compared to monopolar electrosurgery in porcine myometrium. Journal of minimally invasive gynecology, 21(6):11031109, 2014.

[31] Paul K Tulikangas, Tamara Smith, Tommaso Falcone, Navdeep Boparai, and Mark D Walters. Gross and histologic characteristics of laparoscopic injuries with four different energy sources. Fertility and sterility, 75(4):806-810, 2001.

[32] Duncan F Hanby, Grayson Gremillion, Arthur W Zieske, Bridget Loehn, Richard Whitworth, Tamir Wolf, Anagha C Kakade, and Rohan R Walvekar. Harmonic scalpel versus flexible $\mathrm{CO}_{2}$ laser for tongue resection: a histopathological analysis of thermal damage in human cadavers. World journal of surgical oncology, 9(1):83, 2011.

[33] Leonardo S Mattos, Nikhil Deshpande, Giacinto Barresi, Luca Guastini, and Giorgio Peretti. A novel computerized surgeon-machine interface for robotassisted laser phonomicrosurgery. The Laryngoscope, 124(8):1887-1894, 2014.

[34] Tjeerd de Boorder, Laurien Waaijer, Paul J van Diest, and Arjen J Witkamp. Ex vivo feasibility study of endoscopic intraductal laser ablation of the breast. Lasers in Surgery and Medicine, 2017.

[35] Roberto Pini, Renzo Salimbeni, Matteo Vannini, Roberto Barone, and Carlo Clauser. Laser dentistry: a new application of excimer laser in root canal therapy. Lasers in surgery and medicine, 9(4):352-357, 1989. 
[36] Mun Young Chang, Hyun Seok Choi, Sang-Youp Lee, and Ja-Won Koo. Comparison of free-beam-and fiber-type $\mathrm{CO}_{2}$ laser delivery systems in stapes surgery. Journal of Audiology \& Otology, 21(2):103, 2017.

[37] Carl E Silver, Jonathan J Beitler, Ashok R Shaha, Alessandra Rinaldo, and Alfio Ferlito. Current trends in initial management of laryngeal cancer: the declining use of open surgery. European Archives of Oto-Rhino-Laryngology, 266(9):1333$1352,2009$.

[38] Troy Gianduzzo, Jose R Colombo, Georges-Pascal Haber, Jason Hafron, Cristina Magi-Galluzzi, Monish Aron, Inderbir S Gill, and Jihad H Kaouk. Laser robotically assisted nerve-sparing radical prostatectomy: a pilot study of technical feasibility in the canine model. BJU international, 102(5):598-602, 2008.

[39] Kanako Harada, Zhang Bo, Shin Enosawa, Toshio Chiba, and Masakatsu G Fujie. Bending laser manipulator for intrauterine surgery and viscoelastic model of fetal rat tissue. In Robotics and Automation, 2007 IEEE International Conference on, pages 611-616. IEEE, 2007.

[40] Hiromasa Yamashita, Kiyoshi Matsumiya, Ken Masamune, Hongen Liao, Tosho Chiba, and Takeyoshi Dohi. Miniature bending manipulator for fetoscopic intrauterine laser therapy to treat twin-to-twin transfusion syndrome. Surgical endoscopy, 22(2):430-435, 2008.

[41] M Stuart Strong and Geza J Jako. Laser surgery in the larynx early clinical experience with continuous $\mathrm{CO}_{2}$ laser. Annals of Otology, Rhinology \& Laryngology, 81(6):791-798, 1972.

[42] Maren Kleine-Brüggeney, Robert Greif, P Schoettker, Georges Louis Savoldelli, Sabine Nabecker, and Lorenz Gregor Theiler. Evaluation of six videolaryngoscopes in 720 patients with a simulated difficult airway: a multicentre randomized controlled trial. BJA: British Journal of Anaesthesia, 116(5):670-679, 2016.

[43] Nikhil Deshpande, Leonardo S Mattos, Giacinto Barresi, Andrea Brogni, Giulio Dagnino, Luca Guastini, Giorgio Peretti, and Darwin G Caldwell. Imaging based metrics for performance assessment in laser phonomicrosurgery. In Robotics and Automation (ICRA), 2013 IEEE International Conference on, pages 4850-4856. IEEE, 2013.

[44] Snehal Patel, Milind Rajadhyaksha, Stefan Kirov, Yongbiao Li, and Ricardo Toledo-Crow. Endoscopic laser scalpel for head and neck cancer surgery. In SPIE BiOS, pages 82071S-82071S. International Society for Optics and Photonics, 2012.

[45] Rupert Renevier, Brahim Tamadazte, Kanty Rabenorosoa, Laurent Tavernier, and Nicolas Andreff. Endoscopic laser surgery: Design, modeling, and control. IEEE/ASME Transactions on Mechatronics, 22(1):99-106, 2017. 
[46] Nikhil Deshpande, Jesús Ortiz, Darwin G Caldwell, and Leonardo S Mattos. Enhanced computer-assisted laser microsurgeries with a virtual microscope based surgical system. In Robotics and Automation (ICRA), 2014 IEEE International Conference on, pages 4194-4199. IEEE, 2014.

[47] Grzegorz S Litynski. Endoscopic surgery: the history, the pioneers. World journal of surgery, 23(8):745-753, 1999.

[48] Demetrios N Moris, Konstantinos J Bramis, Eleftherios I Mantonakis, Efstathios L Papalampros, Athanasios S Petrou, and Alexandros E Papalampros. Surgery via natural orifices in human beings: yesterday, today, tomorrow. The American Journal of Surgery, 204(1):93-102, 2012.

[49] Richard J Hendrick, S Duke Herrell, and Robert J Webster. A multi-arm handheld robotic system for transurethral laser prostate surgery. In Robotics and Automation (ICRA), 2014 IEEE International Conference on, pages 2850-2855. IEEE, 2014.

[50] [Online] Available: https://www.sages.org/meetings/annual-meeting/abstractsarchive/transgastric-cholecystectomy-using-the-endosamurai-a-novelendoscopic-operating-platform/. Accessed on 27-03-2017.

[51] [Online] Available: https://www.sages.org/meetings/annual-meeting/abstractsarchive/ddes-flexible-single-incision-operating-platform-facilitates-an-extendedthoracic-lymphadenectomy-via-a-trans-hiatal-approach/. Accessed on 27-032017.

[52] Soo Jay Phee, Soon Chiang Low, VA Huynh, Andy Prima Kencana, ZL Sun, and $\mathrm{K}$ Yang. Master and slave transluminal endoscopic robot (master) for natural orifice transluminal endoscopic surgery (notes). In Engineering in Medicine and Biology Society, 2009. EMBC 2009. Annual International Conference of the IEEE, pages 1192-1195. IEEE, 2009.

[53] Daniel J Abbott, Chris Becke, Richard I Rothstein, and William J Peine. Design of an endoluminal notes robotic system. In Intelligent Robots and Systems, $200 \%$. IROS 200\%. IEEE/RSJ International Conference on, pages 410-416. IEEE, 2007.

[54] Parag W Dhumane, Michele Diana, Joel Leroy, and Jacques Marescaux. Minimally invasive single-site surgery for the digestive system: a technological review. Journal of minimal access surgery, 7(1):40, 2011.

[55] Charles Fisher, David Braun, Joel Kaluzny, and Todd Haran. Cobra: A twodegree of freedom fiber optic positioning mechanism. In Aerospace conference, 2009 IEEE, pages 1-11. IEEE, 2009.

[56] Cameron M Lee, Christoph J Engelbrecht, Timothy D Soper, Fritjof Helmchen, and Eric J Seibel. Scanning fiber endoscopy with highly flexible, 1-mm catheterscopes for wide-field, full-color imaging. Journal of biophotonics, 3(5-6):385, 2010. 
[57] Leonardo S Mattos, Darwin G Caldwell, Giorgio Peretti, Francesco Mora, Luca Guastini, and Roberto Cingolani. Microsurgery robots: addressing the needs of high-precision surgical interventions. Swiss Med Wkly, 146:w14375, 2016.

[58] Marc Remacle, Vyas MN Prasad, Georges Lawson, Laetitia Plisson, Vincent Bachy, and Sebastien Van der Vorst. Transoral robotic surgery (tors) with the medrobotics flex system: first surgical application on humans. European Archives of Oto-Rhino-Laryngology, 272(6):1451-1455, 2015.

[59] Andrea Bajo, Roger E Goldman, Long Wang, Dennis Fowler, and Nabil Simaan. Integration and preliminary evaluation of an insertable robotic effectors platform for single port access surgery. In Robotics and Automation (ICRA), 2012 IEEE International Conference on, pages 3381-3387. IEEE, 2012.

[60] Nabil Simaan, Kai Xu, Wei Wei, Ankur Kapoor, Peter Kazanzides, Russell Taylor, and Paul Flint. Design and integration of a telerobotic system for minimally invasive surgery of the throat. The International journal of robotics research, 28(9):1134-1153, 2009.

[61] Dennis Kundrat, Andreas Schoob, Benjamin Munske, and Tobias Ortmaier. Towards an endoscopic device for laser-assisted phonomicrosurgery. In Proceedings of the Hamlyn symposium on medical, robotics, pages 55-56, 2013.

[62] Dennis Kundrat, Andreas Schoob, Lüder A Kahrs, and Tobias Ortmaier. 22 flexible robot for laser phonomicrosurgery. Soft Robotics: Transferring Theory to Application, page 265, 2015.

[63] Kanty Rabenorosoa, Bastien Tasca, Antonin Zerbib, Patrick Rougeot, Nicolas Andreff, and Ton Eakkachai Pengwang. Squipabot: A mesoscale parallel robot for a laser phonosurgery. International Journal of Optomechatronics, 9(4):310$324,2015$.

[64] Digital Acublade System. Lumenis Inc. Israel. [Online] Available: http://www . lumenis.com/Surgical. Accessed on 14-03-2017.

[65] HiScan Surgical. Deka Laser. [Online] Available: http://www.dekalaser.com/ it-IT/accessorynew . aspx?item=smatxidehs\&acc=acc106_SmartxideHS. Accessed on 28-03-2017.

[66] Helena Hotz Arroyo, Larissa Neri, Carina Yuri Fussuma, and Rui Imamura. Diode laser for laryngeal surgery: a systematic review. International archives of otorhinolaryngology, 20(02):172-179, 2016.

[67] Nathaniel M Fried and Keith E Murray. High-power thulium fiber laser ablation of urinary tissues at $1.94 \mu \mathrm{m}$. Journal of endourology, 19(1):25-31, 2005.

[68] Mitchell N Faddis and Bruce D Lindsay. Magnetic catheter manipulation. Coronary artery disease, 14(1):25-27, 2003. 
[69] Ilker Tunay. Position control of catheters using magnetic fields. In Mechatronics, 2004. ICM'04. Proceedings of the IEEE International Conference on, pages 392397. IEEE, 2004.

[70] Klaas Jelmer Boskma, Stefano Scheggi, and Sarthak Misra. Closed-loop control of a magnetically-actuated catheter using two-dimensional ultrasound images. In Biomedical Robotics and Biomechatronics (BioRob), 2016 6th IEEE International Conference on, pages 61-66. IEEE, 2016.

[71] Leonardo S Mattos, Giulio Dagnino, Gabriele Becattini, Massimo Dellepiane, and Darwin G Caldwell. A virtual scalpel system for computer-assisted laser microsurgery. In Intelligent Robots and Systems (IROS), 2011 IEEE/RSJ International Conference on, pages 1359-1365. IEEE, 2011.

[72] Giacinto Barresi, Nikhil Deshpande, Leonardo S Mattos, Andrea Brogni, Luca Guastini, Giorgio Peretti, and Darwin G Caldwell. Comparative usability and performance evaluation of surgeon interfaces in laser phonomicrosurgery. In Intelligent Robots and Systems (IROS), 2013 IEEE/RSJ International Conference on, pages 3610-3615. IEEE, 2013.

[73] Arduino Due Control Board. [Online] Available: https://store.arduino.cc/ usa/arduino-due. Accessed on 08-08-2017.

[74] François Aguet, Dimitri Van De Ville, and Michael Unser. Model-based 2.5$\mathrm{d}$ deconvolution for extended depth of field in brightfield microscopy. IEEE Transactions on Image Processing, 17(7):1144-1153, 2008.

[75] Robert A Schill. General relation for the vector magnetic field of a circular current loop: a closer look. IEEE Transactions on Magnetics, 39(2):961-967, 2003.

[76] William Ralph Smythe. Static and dynamic electricity. Taylor \& Francis, 1988.

[77] John Brooke. Sus-a quick and dirty usability scale. Usability evaluation in industry, 189(194):4-7, 1996.

[78] Mats Lind and Henning Sundvall. Time estimation as a measure of mental workload. In International Conference on Engineering Psychology and Cognitive Ergonomics, pages 359-365. Springer, 2007.

[79] Sabrina Garofolo, Cesare Piazza, Francesca Del Bon, Stefano Mangili, Luca Guastini, Francesco Mora, Piero Nicolai, and Giorgio Peretti. Intraoperative narrow band imaging better delineates superficial resection margins during transoral laser microsurgery for early glottic cancer. Annals of Otology, Rhinology \& Laryngology, 124(4):294-298, 2015. 
[80] Hagit Shoffel-Havakuk, Yonatan Lahav, Erez Shmuel Davidi, Yaara Haimovich, Moshe Hain, and Doron Halperin. The role of separate margins sampling in endoscopic laser surgery for early glottic cancer. Acta oto-laryngologica, 136(5):491496, 2016.

[81] Wolfgang Steiner and Petra Ambrosch. Endoscopic laser surgery of the upper aerodigestive tract: with special emphasis on cancer surgery. Thieme, 2000.

[82] Neil G Hockstein, J Paul Nolan, Bert W O’Malley, and Y Joseph Woo. Robotic microlaryngeal surgery: a technical feasibility study using the davinci surgical robot and an airway mannequin. The Laryngoscope, 115(5):780-785, 2005.

[83] Carlos M Rivera-Serrano, Paul Johnson, Brett Zubiate, Richard Kuenzler, Howie Choset, Marco Zenati, Stephen Tully, and Umamaheswar Duvvuri. A transoral highly flexible robot. The Laryngoscope, 122(5):1067-1071, 2012.

[84] Kevin Olds, Alexander T Hillel, Elizabeth Cha, Martin Curry, Lee M Akst, Russell H Taylor, and Jeremy D Richmon. Robotic endolaryngeal flexible (roboelf) scope: A preclinical feasibility study. The Laryngoscope, 121(11):2371-2374, 2011.

[85] Jessica Burgner, M Müller, Jörg Raczkowsky, and Heinz Wörn. Ex vivo accuracy evaluation for robot assisted laser bone ablation. The International Journal of Medical Robotics and Computer Assisted Surgery, 6(4):489-500, 2010.

[86] Lüder A Kahrs, Jessica Burgner, Thomas Klenzner, Jörg Raczkowsky, Jörg Schipper, and Heinz Wörn. Planning and simulation of microsurgical laser bone ablation. International Journal of Computer Assisted Radiology and Surgery, 5(2):155-162, 2010.

[87] Ben Y C Leung, Paul J L Webster, James M Fraser, and Victor X D Yang. Realtime guidance of thermal and ultrashort pulsed laser ablation in hard tissue using inline coherent imaging. Lasers in Surgery and Medicine, 44(3):249-256, 2012.

[88] Sebastian Stopp, Daniel Svejdar, Emanuel Von Kienlin, Herbert Deppe, and Tim C Lueth. A new approach for creating defined geometries by navigated laser ablation based on volumetric 3-d data. Biomedical Engineering, IEEE Transactions on, 55(7):1872-1880, 2008.

[89] Erwin Bay, Xosè Luis Deán-Ben, Genny A. Pang, Alexandre Douplik, and Daniel Razansky. Real-time monitoring of incision profile during laser surgery using shock wave detection. Journal of Biophotonics, 2013.

[90] Loris Fichera, Diego Pardo, Placido Illiano, Darwin G Caldwell, and Leonardo S Mattos. Feed forward incision control for laser microsurgery of soft tissue. In Robotics and Automation (ICRA), 2015 IEEE International Conference on, pages 1235-1240. IEEE, 2015. 
[91] Kawe Goharkhay, Andreas Moritz, Petra Wilder-Smith, Ulrich Schoop, Wolf Kluger, Sabine Jakolitsch, and Wolfgang Sperr. Effects on oral soft tissue produced by a diode laser in vitro. Lasers in surgery and medicine, 25(5):401-406, 1999.

[92] Millard M Judy, J Lester Matthews, Billie L Aronoff, and Donald F Hults. Soft tissue studies with $805 \mathrm{~nm}$ diode laser radiation: thermal effects with contact tips and comparison with effects of $1064 \mathrm{~nm}$ nd: Yag laser radiation. Lasers in surgery and medicine, 13(5):528-536, 1993.

[93] Markolf Niemz. Laser-tissue Interactions. Springer Berlin Heidelberg, 2004.

[94] Loris Fichera, Diego Pardo, Placido Illiano, Jesùs Ortiz, Darwin G Caldwell, and Leonardo S Mattos. Online estimation of laser incision depth for transoral microsurgery: approach and preliminary evaluation. The International Journal of Medical Robotics and Computer Assisted Surgery, 12(1):53-61, 2016.

[95] Petra Wilder-Smith, Anna-Marie A Arrastia, Lih-Hueh Liaw, and Michael Berns. Incision properties and thermal effects of three $\mathrm{CO}_{2}$ lasers in soft tissue. Oral Surgery, Oral Medicine, Oral Pathology, Oral Radiology, and Endodontology, 79(6):685-691, 1995.

[96] Steven L Jacques. Optical properties of biological tissues: a review. Physics in Medicine and Biology, 58(11):R37, 2013.

[97] Loris Fichera, Claudio Pacchierotti, Emidio Olivieri, Domenico Prattichizzo, and Leonardo S Mattos. Kinesthetic and vibrotactile haptic feedback improves the performance of laser microsurgery. In 2016 IEEE Haptics Symposium (HAPTICS), pages 59-64. IEEE, 2016.

[98] Alfonso Fiorelli, Salvatore Mazzone, Adriano Mazzone, and Mario Santini. The digital acublade laser system to remove huge vocal fold granulations following subglottic airway stent. Interactive cardiovascular and thoracic surgery, 17(3):591-593, 2013. 